\title{
Quantum homology of fibrations over $S^{2}$
}

\author{
Dusa McDuff* \\ State University of New York at Stony Brook \\ (dusa@math.sunysb.edu)
}

May 12, 1999

\begin{abstract}
This paper studies the (small) quantum homology and cohomology of fibrations $p: P \rightarrow$ $S^{2}$ whose structural group is the group of Hamiltonian symplectomorphisms of the fiber $(M, \omega)$. It gives a proof that the rational cohomology splits additively as the vector space tensor product $H^{*}(M) \otimes H^{*}\left(S^{2}\right)$, and investigates conditions under which the ring structure also splits, thus generalizing work of Lalonde-McDuff-Polterovich and Seidel. The main tool is a study of certain operations in the quantum homology of the total space $P$ and of the fiber $M$, whose properties reflect the relations between the Gromov-Witten invariants of $P$ and $M$. In order to establish these properties we further develop the language introduced in $[\mathrm{Mc} 3]$ to describe the virtual moduli cycle (defined by Liu-Tian, Fukaya-Ono, Li-Tian, Ruan and Siebert).
\end{abstract}

AMS classification number 53C15;

key words: quantum cohomology, symplectic fibration, Hamiltonian fibration, GromovWitten invariants

*Partially supported by NSF grant DMS 9704825. 


\section{Main results}

\subsection{The homology of $P$}

This paper studies the quantum homology of Hamiltonian fibrations

$$
p: P \rightarrow S^{2}
$$

A fibration $P \rightarrow B$ is called symplectic if its fiber is a symplectic manifold $(M, \omega)$ and its structural group is the group of $\operatorname{symplectomorphisms} \operatorname{Symp}(M, \omega)$. Thus each fiber $M_{b}=p^{-1}(b)$ is equipped with a well-defined symplectic form $\omega_{b}$. To say that $p$ is Hamiltonian means that the structural group reduces further to the group of Hamiltonian symplectomorphisms $\operatorname{Ham}(M, \omega)$. As noted by Seidel $[\mathrm{Sd} 1]$, when $B=S^{2}$ this is equivalent to the existence of a symplectic form $\Omega$ on $P$ that restricts to $\omega_{b}$ on each fiber. Moreover, we can choose $\Omega$ to be compatible with the orientation of $P$ defined by the standard orientations on $S^{2}$ and on $(M, \omega)$. Such forms $\Omega$ are said to be compatible with $p$. It is easy to see that the set of all such forms is path connected. This means in particular that the Gromov-Witten invariants of $P$ do not depend on the choice of $\Omega$.

We shall say that the rational cohomology $H^{*}(P)=H^{*}(P, \mathbf{Q})$ of $P$ is additively split if it is isomorphic (as a vector space) to the tensor product $H^{*}(M) \otimes H^{*}(B)$. This happens if and only if the rational homology $H_{*}(M)$ of $M$ injects into that of $P$. The first aim of this paper is to give a proof of the following result that was announced in Lalonde-McDuffPolterovich [LMP2]. It has several interesting corollaries related to the Flux conjecture for $(M, \omega)$ that are fully described there. (Cf also [LMP1].) The extent to which it generalises to Hamiltonian fibrations with other bases is discussed in the forthcoming paper [LMP3].

Theorem 1.1 The rational cohomology of any Hamiltonian fibration with base $S^{2}$ is additively split.

The reason for this is that each such fibration gives rise to an operation on the quantum homology $Q H_{*}(M)$ of $(M, \omega)$, whose existence implies that the rational homology $H_{*}(M)$ of $M$ injects into that of $P$. This operation was first investigated by Seidel in [Sd1] and has the form

$$
a \mapsto Q_{\sigma} *_{M} a, \quad a \in H_{*}(M, \mathbf{R}),
$$

where $\sigma \in H_{2}(P, \mathbf{Z})$ is a reference section, $Q_{\sigma} \in Q H_{*}(M)$ represents the intersection with $M$ of all $J$-holomorphic sections of $P \rightarrow M$, and $*_{M}$ is the quantum product in $M$. For more details see $\S \S 1.3,2$. Note that we consider $Q_{\sigma}$ as an element of $Q H_{*}(M)$ rather than $H_{*}(M)$ in order to keep track of the homology classes of the sections considered.

The above theorem was proved by Deligne when $p$ is a holomorphic fibration with Kähler total space. When the the loop in $\operatorname{Ham}(M)$ that generates the fibration comes from a circle action, it also follows from work of Kirwan on equivariant cohomology. (We show in Example 2.7 that not all loops have this form.) More generally, Polterovich pointed out that Blanchard [B] found an easy cohomological proof that applies in the case of smooth fibrations with fiber of "hard Lefschetz type." " Also, it was proved in [LMP2] in cases where the "usual" theory of $J$-holomorphic spheres works, for example, if $(M, \omega)$ is spherically monotone. In the present paper the "new" theory of Gromov-Witten invariants (due to

\footnotetext{
${ }^{1}$ This means that the map $\wedge\left[\omega^{k}\right]: H^{n-k}(M, \mathbf{R}) \rightarrow H^{n+k}(M, \mathbf{R})$ is an isomorphism for all $k$.
} 
Fukaya-Ono [FO], Li-Tian [LiT], Liu-Tian $[\mathrm{LiuT}]$, Ruan $[\mathrm{R}]$ and Siebert $[\mathrm{S}]$ ) is used to extend the result to arbitrary $(M, \omega)$. In order to do this, we adapt this theory to our fibered setting in order to show that the operation we define has the requisite properties: see $\S 1.3, \S 2$, and $\S 4$.

Thus the additive structure of $H^{*}(P)$ is always very simple. The story concerning its multiplicative structure is more complicated since one can consider both the standard cup product and also versions of the quantum (or deformed) cup product. For example, Seidel exploits properties of the quantum product in his work on $\operatorname{Symp}\left(\mathbf{C P} \mathbf{P}^{m} \times \mathbf{C P}^{n}\right)$ in [Sd2]. He also pointed out that, if there is a fibered $\Omega$-tame almost complex structure $J$ on $P$ that admits no $J$-holomorphic spheres in any fiber, then $H^{*}(P)$ is isomorphic as a ring (under cup product) with the product of the rings $H^{*}\left(S^{2}\right)$ and $H^{*}(M)$. For example, this would be the case if $\omega=0$ on $\pi_{2}(M)$. However, it is certainly not true that the ring $H^{*}(P)$ always splits in this way: see Example 3.12.

We now state a generalization of this result with hypothesis framed in terms of the vanishing of certain Gromov-Witten invariants. Given any symplectic manifold $(X, \omega)$ we denote by $n_{X}\left(v_{1}, \ldots, v_{k} ; B\right)$ the Gromov-Witten invariant that counts the number of isolated $J$-holomorphic spheres in class $B \in H_{2}(X, \mathbf{Z})$ that intersect representing cycles of the homology classes $v_{1}, \ldots, v_{k} \in H_{*}(X)$. (For more details on these invariants, see $\S \S 1.3,2,4$.) We shall denote the rational cohomology of $X$ by $H^{*}(X)$ and shall write $\iota: H_{*}(M) \rightarrow H_{*}(P)$ for the map induced by the inclusion of $M$ as a fiber.

Theorem 1.2 Let $(M, \omega) \rightarrow P \rightarrow S^{2}$ be a fibration with structural group $\operatorname{Ham}(M, \omega)$, and suppose that the Gromov-Witten invariants

$$
n_{P}\left(v_{1}, \ldots, v_{k} ; \iota(B)\right), \quad v_{j} \in H_{*}(P), B \neq 0, k \leq 4,
$$

vanish. Then $H^{*}(P)$ is isomorphic as a ring under cup product with the product of the rings $H^{*}\left(S^{2}\right)$ and $H^{*}(M)$.

Other conditions under which the ring splits are stated in Proposition 3.23. However, the validity of the suggestion made in [Mc4] that the ring splits under the sole assumption that the quantum product in $M$ is trivial is not yet clear.

Our approach also allows us to find conditions under which the nonsqueezing theorem holds for the fibration $P \rightarrow S^{2}$. By this we mean the following. Let $\Omega$ be a symplectic form on $P$ that is compatible with the fibration $P \rightarrow S^{2}$ and define the area of $(P, \Omega)$ to be the number $\alpha$ such that

$$
\frac{1}{(n+1) !} \int_{P} \Omega^{n+1}=\frac{\alpha}{n !} \int_{M} \omega^{n} .
$$

Thus, if the fibration $P \rightarrow S^{2}$ is symplectically trivial so that $(P, \Omega)$ is the product $(M \times$ $\left.S^{2}, \omega \oplus \omega_{S}\right), \alpha$ is simply the area of the base $\left(S^{2}, \omega_{S}\right)$. T. Then we will say that the nonsqueezing theorem holds for the fibration $p:(P, \Omega) \rightarrow S^{2}$ if the area $\alpha$ constrains the size of the balls

\footnotetext{
${ }^{2}$ Private communication

3 i.e. it restricts on each fiber to an $\omega$-tame almost complex structure: see Definition 2.8 for a more precise definition

${ }^{4}$ Polterovich showed in $[\mathrm{P}]$ that the minimum of the areas of all compatible symplectic forms on $P$ is an interesting measurement of the size of the generating loop $\phi$ of $P$ (see $\S 1.2$ ) that is a kind of "one-sided" Hofer norm for $\phi$.
} 
that embed into $(P, \Omega)$, i.e. if $\pi r^{2} \leq \alpha$ whenever $B^{2 n+2}(r)$ embeds symplectically in $(P, \Omega)$. By considering the case when $(P, \Omega)$ is $\mathbf{C} \mathbf{P}^{2}$ blown up at a point with one of the standard symplectic forms, it is not hard to see that the nonsqueezing theorem does not hold for all $(P, \Omega)$.

The best result that our methods give about the nonsqueezing theorem is stated in $\S 3.4$ as Proposition 3.27. Here is a corollary.

Proposition 1.3 Suppose that the hypotheses of Theorem 1.9 hold. Then, the nonsqueezing theorem holds for the fibration $p:(P, \Omega) \rightarrow S^{2}$.

\subsection{Homomorphisms defined on $\pi_{1}(\operatorname{Ham}(M, \omega))$}

In this section we discuss three homomorphisms defined on $\pi_{1}(\operatorname{Ham}(M, \omega))$ that measure the cohomological twisting of the fibration $P \rightarrow S^{2}$. The most significant one is adapted from Seidel [Sd1] and is assembled from some of the "horizontal" Gromov-Witten invariants of $P$.

First, recall that any bundle over $S^{2}$ can be constructed by taking product bundles over two discs and gluing them by a clutching function $\phi=\left\{\phi_{t}\right\}_{t \in[0,1]}$, viz:

$$
P_{\phi}=\left(D_{+}^{2} \times M\right) \cup_{\phi}\left(D_{-}^{2} \times M\right),
$$

where

$$
\phi:(2 \pi t, x)_{+} \mapsto\left(-2 \pi t, \phi_{t}(x)\right)_{-} .
$$

(The orientation on the base is induced from that on $D_{+}^{2}$.) Moreover if we fix an identification of one fiber of $P$ with $M$, we can normalise $\left\{\phi_{t}\right\}$ by requiring that $\phi_{0}=$ id and get a loop that is well defined up to homotopy. It is not hard to see that $P$ is a Hamiltonian fibration precisely when $\phi$ is homotopic to a $\operatorname{loop}$ in $\operatorname{Ham}(M, \omega)$. Thus there is a bijective correspondence between loops $\phi \in \pi_{1}(\operatorname{Ham}(M, \omega))$ and Hamiltonian fibrations $P_{\phi}$ with one fiber identified with $M$.

As noted in [LMP2], the manifold $P_{\phi}$ carries two canonical cohomology classes, the first Chern class of the vertical tangent bundle

$$
c_{\phi}=c_{1}\left(T P_{\phi}^{\text {vert }}\right) \in H^{2}\left(P_{\phi}, \mathbf{Z}\right),
$$

and the coupling class $u_{\phi}$, i.e. the unique class in $H^{2}\left(P_{\phi}, \mathbf{R}\right)$ such that

$$
i^{*}\left(u_{\phi}\right)=[\omega], \quad u_{\phi}^{n+1}=0 .
$$

We will see in Lemma 2.4 that these classes behave well under composition of loops.

Since these classes are canonical, they give rise to homomorphisms from $\pi_{1}(\operatorname{Ham}(M, \omega))$. The first, defined by Seidel in [Sd1] 110 , has the form

$$
\bar{I}_{c}: \pi_{1}(\operatorname{Ham}(M, \omega)) \rightarrow \mathbf{Z} / N \mathbf{Z}
$$

where $N$ is the minimal spherical Chern number, i.e. the smallest nonnegative integer such that $c_{1}: H_{2}^{S}(M, \mathbf{Z}) \rightarrow \mathbf{Z}$ takes values in $N \mathbf{Z}$. (Here $H_{2}^{S}(M, \mathbf{Z})$ be the spherical part of $H_{2}(M)$, that is the image of the Hurewicz homomorphism $\pi_{2}(M) \rightarrow H_{2}(M, \mathbf{Z})$.) In our language, $\bar{I}_{c}$ is defined as

$$
\bar{I}_{c}(\phi)=c_{\phi}(\sigma) \quad(\bmod N),
$$


where $\sigma$ is any section class of $P_{\phi}$. This is well defined since $c_{\phi}\left(\sigma-\sigma^{\prime}\right) \in N \mathbf{Z}$ by definition of $N$.

The second homomorphism is determined by the Poincaré dual $\operatorname{PD}\left(u_{\phi}^{n}\right) \in H_{2}\left(P_{\phi}, \mathbf{R}\right)$. Polterovich pointed out that there is a canonical identification between the quotients $H_{2}(P, \mathbf{R}) / H_{2}^{S}(P, \mathbf{R})$ and $H_{2}(M, \mathbf{R}) / H_{2}^{S}(M, \mathbf{R})$. Moreover, both groups are isomorphic to the second homology group $H_{2}\left(\pi_{1}(M), \mathbf{R}\right)$ of $\pi_{1}(M)$. (This follows by looking at the homology spectral sequence of the fibration $\widetilde{M} \rightarrow M \rightarrow B\left(\pi_{1}(M)\right)$ where $\widetilde{M}$ is the universal cover of $M$ and $B G$ denotes the classifying space of the group $G$.) Hence there is a map

$$
\bar{I}_{u}: \pi_{1}(\operatorname{Ham}(M)) \rightarrow H_{2}\left(\pi_{1}(M), \mathbf{R}\right): \quad \phi \mapsto\left[\mathrm{PD}\left(u_{\phi}^{n}\right)\right]
$$

and it follows from Lemma 2.4 that this is a homomorphism. By Example 3.12 below, this homomorphism is not always trivial.

Remark 1.4 It would be interesting to define these homomorphisms in a more intrinsic way that involved only $M$, not $P_{\phi}$. Since the definition of $\bar{I}_{c}$ depends only on the fact that the bundle $P \rightarrow S^{2}$ has a well defined fiberwise almost structure, $\bar{I}_{c}$ extends to a homomorphism defined on the kernel of the homomorphism $\pi_{1}(\operatorname{Diff}(M)) \rightarrow \pi_{1}(\mathcal{A})$ where $\mathcal{A}$ is the space of all almost complex structures on $M$ that are homotopic to an $\omega$-tame $J$. Similarly, one can extend the domain of $\bar{I}_{u}$ to the kernel of the generalized flux homomorphism $F$ : $\pi_{1}(\operatorname{Diff}(M)) \rightarrow H^{1}(M, \mathbf{R})$

$$
F(\phi)(\gamma)=\left\langle\omega, \phi_{*}(\gamma)\right\rangle
$$

where $\phi_{*}(\gamma)$ is the trace $T^{2} \rightarrow M:(s, t) \mapsto \phi_{t}(\gamma(s))$ under $\phi$ of the loop $\gamma(s)$. This holds because $F(\phi)=0$ precisely when there is a cohomology class in $H^{2}\left(P_{\phi}\right)$ that restricts to $[\omega]$, and this is the assumption underlying the definition of the coupling class $u_{\phi}$. A family $I_{k}$ of homomorphisms closely related to $\bar{I}_{u}$ was defined in Corollary 3.F of [LMP2] as follows:

$$
I_{k}: \pi_{1}\left(\operatorname{Ham}(M) \rightarrow \mathbf{R}: \quad \phi \rightarrow \int_{P_{\phi}}\left(c_{\phi}\right)^{k}\left(u_{\phi}\right)^{n+1-k} .\right.
$$

In particular, $I_{1}(\phi)=c_{\phi}\left(\mathrm{PD}\left(\left(u_{\phi}\right)^{n}\right)\right.$.

Next, we define a version of the small quantum homology $Q H_{*}\left(M, \Lambda_{R}\right)$ with coefficients in a real Novikov ring $\Lambda_{R}$. Set $c=c_{1}(T M) \in H^{2}(M, \mathbf{Z})$. Let $\Lambda$ be the usual Novikov ring of the group $\mathcal{H}=H_{2}^{S}(M, \mathbf{Z}) / \sim$ with valuation $I_{\omega}$ where $B \sim B^{\prime}$ if $\omega\left(B-B^{\prime}\right)=c\left(B-B^{\prime}\right)=0$, and let $\Lambda_{R}$ be the analogous (real) Novikov ring based on the group $\mathcal{H}_{R}=H_{2}^{S}(M, \mathbf{R}) / \sim$. Thus $\Lambda$ is the completion of the group ring of $\mathcal{H}$ with elements of the form

$$
\sum_{B \in \mathcal{H}} \lambda_{B} e^{B}
$$

where for each $\kappa$ there are only finitely many nonzero $\lambda_{B} \in \mathbf{Q}$ with $\omega(B)>-\kappa$. Similarly, the elements of $\Lambda_{R}$ are

\footnotetext{
${ }^{5}$ Private communication
}

$$
\sum_{B \in \mathcal{H}_{R}} \lambda_{B} e^{B},
$$


where $\lambda_{B} \in \mathbf{Q}$ and there is a similar finiteness condition. Set

$$
Q H_{*}(M)=H_{*}(M) \otimes \Lambda, \quad Q H_{*}\left(M, \Lambda_{R}\right)=H_{*}(M) \otimes \Lambda_{R} .
$$

Then $Q H_{*}(M)$ is $\mathbf{Z}$-graded with $\operatorname{deg}\left(a \otimes e^{B}\right)=\operatorname{deg}(a)+2 c_{1}(B)$. It is best to think of $Q H_{*}\left(M, \Lambda_{R}\right)$ as $\mathbf{Z} / 2 \mathbf{Z}$-graded with

$$
Q H_{e v}=H_{e v}(M) \otimes \Lambda_{R}, \quad Q H_{o d d}=H_{\text {odd }}(M) \otimes \Lambda_{R} .
$$

Recall that the quantum intersection product

$$
a *_{M} b \in Q H_{i+j-2 n}(M), \quad \text { for } a \in H_{i}(M), b \in H_{j}(M)
$$

is defined as follows:

$$
a *_{M} b=\sum_{B \in \mathcal{H}}\left(a *_{M} b\right)_{B} \otimes e^{-B},
$$

where $\left(a *_{M} b\right)_{B} \in H_{i+j-2 n+2 c(B)}(M)$ is defined by the requirement that

$$
\left(a *_{M} b\right)_{B} \cdot{ }_{M} c=n_{M}(a, b, c ; B) \quad \text { for all } c \in H_{*}(M) .
$$

Here we have written $\cdot_{M}$ for the usual intersection pairing on $H_{*}(M)$. Thus $a \cdot{ }_{M} b=0$ unless $\operatorname{dim}(a)+\operatorname{dim}(b)=2 n$ in which case it is the algebraic number of intersection points of the cycles. The product $*_{M}$ is extended to $Q H_{*}(M)$ by linearity over $\Lambda$, and is associative. Moreover, it preserves the grading if we set

$$
\operatorname{deg} c \otimes e^{B}=\operatorname{dim}(c)+2 c_{1}(B) .
$$

(The above formula is consistent with [Sd1] and is appropriate for quantum homology rather then cohomology.) The product clearly extends also to $\Lambda_{R}$. Note here that when defining $a *_{M} b$ we still sum over classes $B \in \mathcal{H}$ (and not $B \in \mathcal{H}_{R}$ ), since $J$-holomorphic spheres can only represent integral classes.

With respect to this product $*_{M}$, both versions of quantum homology are graded commutative rings with unit $\mathbb{1}=[M]$. Further, the invertible elements in $Q H_{e v}\left(M, \Lambda_{R}\right)$ form a group $Q H_{e v}\left(M, \Lambda_{R}\right)^{\times}$that acts on $Q H_{*}\left(M, \Lambda_{R}\right)$ by quantum multiplication.

The following result is proved in $\S 3$. It is a mild generalization of a result of Seidel [Sd1].

Theorem 1.5 For each symplectic manifold $(M, \omega)$ there is a natural homomorphism

$$
\rho: \pi_{1}(\operatorname{Ham}(M, \omega)) \rightarrow Q H_{e v}\left(M, \Lambda_{R}\right)^{\times} .
$$

In fact $\rho(\phi)=Q_{\sigma}$, where $Q_{\sigma}$ is made from the $J$-holomorphic sections of $P$ as described just after the statement of Theorem 1.1 and $\sigma=\sigma_{\phi}$ is a suitable reference section that is defined in Lemma 3.2. The homomorphism $\rho$ is determined by Gromov-Witten invariants of the form

$$
n_{P}([M],[M], \iota(a) ; \sigma)
$$

where $\iota: H_{*}(M) \rightarrow H_{*}(P)$ is the inclusion and $\sigma$ is a section class.

Seidel showed that $\rho$ is often nontrivial. For example, $\pi_{1}\left(\operatorname{Ham}\left(S^{2}, \omega\right)\right) \cong \pi_{1}(\mathrm{SO}(3))=$ $\mathbf{Z} / 2 \mathbf{Z}$ is generated by a loop $\phi$ that consists of a full turn about some axis and one can check that

$$
\rho(\phi)=[p t] \otimes e^{A / 2}, \quad \text { where } A=\left[S^{2}\right] .
$$


Observe also that if $[\omega]$ is rational one can replace $\mathcal{H}_{R}$ by the corresponding rational group $\mathcal{H}_{Q}$.

We will see in $\S 2.1$ that the very existence of $\rho$ is enough to prove that $H^{*}\left(P_{\phi}\right)$ is additively split for every $\phi$. However, it is not clear if the condition $\rho(\phi)=\mathbb{1}$ guarantees that $H^{*}\left(P_{\phi}\right)$ splits as a ring, though so far there are no counterexamples either. Statement (i) in Proposition 3.23 is as far as we have been able to go in this direction.

\subsection{The fiberwise quantum homology of $P$}

Most of the proofs of the above results are based on the relations between the GromovWitten invariants of $M$ and those of $P_{\phi}$. Here we will state these relations explicitly. In order to put them in a nice form we will suppose in this section that Theorem 1.1 is proven. This implies that the Wang sequence for the homology of $P_{\phi}$ degenerates into the short exact sequence:

$$
0 \rightarrow H_{*+2}(M, \mathbf{R}) \stackrel{\iota}{\rightarrow} H_{*+2}\left(P_{\phi}, \mathbf{R}\right) \stackrel{\cap[M]}{\rightarrow} H_{*}(M, \mathbf{R}) \rightarrow 0,
$$

where $\iota$ is the map induced on homology by the inclusion. Let $s: H_{*}(M, \mathbf{R}) \rightarrow H_{*+2}\left(P_{\phi}, \mathbf{R}\right)$ be any splitting of this sequence. Note the identity:

$$
s(a) \cdot P_{P} \iota(b)=a \cdot{ }_{M} b .
$$

Besides the 3 point Gromov-Witten invariant mentioned above, we consider the 4 point invariant in which the cross ratio of the marked points on the domain is fixed. We denote this by

$$
n_{P, \chi}\left(v_{1}, v_{2}, v_{3}, v_{4} ; D\right) .
$$

Intuitively it counts the number of $J$-holomorphic maps $h: S^{2} \rightarrow P$ in class $D$ such that $h\left(z_{i}\right) \in \nu_{i}, i=1, \ldots, 4$, where $\nu_{i}$ is a cycle representing the class $v_{i}$ and where the cross ratio of the 4 points $z_{1}, \ldots, z_{4}$ on $S^{2}$ is fixed. The following important identity is equivalent to the associativity of quantum multiplication:

$$
n_{P, \chi}\left(v_{1}, \ldots, v_{4} ; A\right)=\sum_{A=A_{1}+A_{2}, \alpha} n_{P}\left(v_{1}, v_{2}, e_{\alpha}^{\prime} ; A_{1}\right) \cdot n_{P}\left(f_{\alpha}^{\prime}, v_{3}, v_{4} ; A_{2}\right)
$$

where $\left\{e_{\alpha}^{\prime}\right\}_{\alpha}$ is a basis for the homology $H_{*}(P, \mathbf{Q})$, and $\left\{f_{\beta}^{\prime}\right\}_{\beta}$ is the dual basis with respect to the intersection pairing. Observe also that often the class $B$ or $D$ is not an individual homology class, but rather is an equivalence class of such: see for example equation (11) above. Whenever it is not clear from the context what is meant, either interpretation will do.

The following proposition expresses the compatibility between the invariants of $P_{\phi}$ and $M$. A similar statement (applied to the fibered space $\mathcal{X}$ with fibers $X_{t}$ ) forms a crucial step in the proof of Theorem 1.1: see $\S$ 2.3.2. A spherical class $\sigma \in H_{2}^{S}(P ; \mathbf{Z})$ is called a section class if $\sigma \cdot[M]=1$.

Proposition 1.6 For any classes $a, b, c \in H_{*}(M), v, w \in H_{*}(P), B \in H_{2}(M, \mathbf{Z})$, any splitting $s$ and any section class $\sigma$ we have:

(i) $n_{P}(\iota(a), \iota(b), v ; \iota(B))=0$. 
(ii) $n_{P}(\iota(a), v, w ; \iota(B))=n_{M}(a, v \cap[M], w \cap[M] ; B)$. In particular

$$
\begin{aligned}
& n_{P}([M], v, w ; \iota(B))=0 \text { if } B \neq 0 ; \\
& n_{P}(\iota(a), s(b), s(c) ; \iota(B))=n_{M}(a, b, c ; B) \text {. }
\end{aligned}
$$

(iii) $n_{P}(\iota(a), \iota(b), v \cap[M] ; \sigma)=n_{P, \chi}(\iota(a), \iota(b), v,[M] ; \sigma)$.

(iv) $\quad n_{P}(v, \iota(a), \iota(b) ; \sigma)$

$$
\begin{aligned}
=\sum_{B, i} n_{P}\left(v,[M], \iota\left(e_{i}\right) ; \sigma-\iota(B)\right) \cdot n_{M}\left(f_{i}, a, b ; B\right) \\
\quad+n_{P}\left([M],[M], \iota\left(e_{i}\right) ; \sigma-\iota(B) \cdot n_{M}\left(f_{i}, v \cap[M], a, b ; B\right)\right.
\end{aligned}
$$

where $\left\{e_{i}\right\}$ is a basis for $H_{*}(M)$ with dual basis $\left\{f_{i}\right\}$.

Although this proposition is very close to well known properties of Gromov-Witten invariants, there do not seem to be any relevant references in the literature. Any expert in the field would no doubt be able to supply a proof: we give one in $§ 4.4 .2$. (iii) and (iv) are especially noteworthy. The latter is an analog of the associativity rule (2) and is derived by moving the representing cycles for $\iota(a), \iota(b)$ into the same fiber instead of moving two of the marked points together. In this case we count stable maps that have two components, one a section in $P$ and the other a vertical curve (i.e. lying in a fiber.) The first sum corresponds to the case when the section meets $v$ and the vertical component meets $a, b$, and the second to the case when the vertical component meets all three cycles $a, b, v \cap[M]$.

Next we introduce a fiberwise quantum product in $P$.

Definition 1.7 Let $Q H_{*}^{V}(P, \Lambda)=H_{*}(P) \otimes \Lambda$, where $\Lambda=\Lambda(M)$ is as before. Given $u, v \in$ $H_{*}(P)$, we define the vertical quantum product in $P$ by setting $u *_{V} v=\sum_{B}\left(u *_{V} v\right)_{B} \otimes e^{-B}$ where

$$
\left(u *_{V} v\right)_{B} \cdot P=n_{P}(u, v, w ; \iota(B)) .
$$

The next result exhibits the relations between the products $*_{M}$ and $*_{V}$.

Lemma 1.8 For all $a, b \in H_{*}(M)$ and any splitting $s$, we have

(i) $\iota(a) *_{V} \iota(b)=0$,

(ii) $s(a) *_{V} \iota(b)=\iota\left(a *_{M} b\right)$,

(iii) $\left(s(a) *_{V} s(b)\right) \cap[M]=a *_{M} b$.

Proof: This can be proved by using Proposition 1.6 and the fact that the class $\iota(a)$ is determined by the identities

$$
\iota(a) \cdot_{P} s(b)=a \cdot_{M} b, \quad \iota(a) \cdot P \iota(b)=0, \quad b \in H_{*}(M) .
$$

Details are left to the reader.

The following quantum analog of the Wang sequence in homology is an immediate consequence. 
Proposition 1.9 The sequence

$$
0 \rightarrow Q H_{*+2}\left(M, \Lambda_{R}\right) \stackrel{\iota}{\rightarrow} Q H_{*+2}^{V}\left(P_{\phi}, \Lambda_{R}\right) \stackrel{\cap[M]}{\rightarrow} Q H_{*}\left(M, \Lambda_{R}\right) \rightarrow 0 .
$$

is a short exact sequence of $Q H_{*}\left(M, \Lambda_{R}\right)$-modules, where the element $a \in Q H_{*}\left(M, \Lambda_{R}\right)$ acts on $Q H_{*}\left(M, \Lambda_{R}\right)$ via $b \mapsto a *_{M} b$ and on $Q H_{*+2}^{V}\left(P_{\phi}, \Lambda_{R}\right)$ via

$$
v \mapsto s(a) *_{V} v .
$$

Note that the module structure on the middle term depends, in principle, on the choice of splitting $s$. There is a dual version of this for cohomology stated in $\S 3.2$ below that generalizes a result in Seidel [Sd2]. In this paper we have chosen to work mostly with homology since this formulation seems to display the geometry most transparently. However, as is evidenced by Seidel's work, in some ways the structure on cohomology is easier to understand.

As mentioned above, the proof of Theorem 1.1 is based on the properties of an operation

$$
\Psi_{\phi, \sigma}: Q H_{*}(M) \rightarrow Q H_{*}(M)
$$

that is associated to the fibration $P_{\phi}$. (Here $\sigma$ is a reference section class in $H_{2}\left(P_{\phi}, \mathbf{Z}\right)$.) One can define this operation in terms of the (first order) horizontal part $*_{H, \sigma}$ of the full quantum multiplication $*_{P}$ on $P_{\phi}$ as follows.

Definition 1.10 Given a reference section $\sigma$, identify the set of all section classes in $H_{2}(P, \mathbf{Z})$ with $H_{2}(M, \mathbf{Z})$ by writing them as $\sigma+\iota(B)$. Then, define the multiplication $*_{H, \sigma}$ on $Q H_{*}^{V}(P)$ by setting $u *_{H, \sigma} v=\sum_{B}\left(u *_{H, \sigma} v\right)_{B} \otimes e^{-B}$ where

$$
\left(u *_{H, \sigma} v\right)_{B} \cdot P=n_{P}(u, v, w ; \sigma+\iota(B)) .
$$

Thus $*_{H, \sigma}$ is exactly that part of $*_{P}$ coming from the section classes, just as $*_{V}$ is the part coming from the fiber classes. One way to define $\Psi_{\phi, \sigma}$ is as:

$$
\Psi_{\phi, \sigma}(a)=\left([M] *_{H, \sigma} \iota(a)\right) \cap[M] .
$$

We will see in $\S 3.1$ that the homomorphism $\rho$ of Theorem 1.5 can be defined by setting

$$
\rho(\phi)=\left([M] *_{H, \sigma_{\phi}}[M]\right) \cap[M]
$$

for a suitable choice of (generalized) section $\sigma_{\phi}$. Moreover, in the situation considered in Theorem 1.2 there is a section class $\sigma_{A}$ with $c_{1}\left(\sigma_{A}\right)=2$ such that the map

$$
a \mapsto s_{A}(a)=[M] *_{H, \sigma_{A}} a
$$

splits the homology ring.

It would of course be very interesting to understand the full quantum (co)homology of $P_{\phi}$. In particular, to what extent is it determined by $Q H^{*}(M)$ and Seidel's homomorphism $\rho$ ? Givental gives in [Gi] some hints as to what an answer might be, at least in the case when $\phi$ comes from a circle action. The work in $\S 3.3$ can be considered as the beginning 
of an attempt to tackle this question: in particular in part (ii) of Proposition 3.23 we show that assumptions on the Gromov-Witten invariants of $M$ and on $\rho$ do give information on the (undeformed) cup product on $H^{*}(P)$. The easiest case is when $P$ is the product $M \times S^{2}$. Kontsevich and Manin [KM] showed (among many other things) that

$$
Q H^{*}\left(M \times S^{2}\right)=Q H^{*}(M) \otimes Q H^{*}\left(S^{2}\right)
$$

when $M$ is a projective algebraic manifold. The next result extends this to all symplectic $M$. This is surely well known, and we include a sketch of the proof for the sake of completeness: see Proposition 3.25 .

Proposition 1.11 For all closed symplectic manifolds $M$,

$$
Q H^{*}\left(M \times S^{2}\right)=Q H^{*}(M) \otimes Q H^{*}\left(S^{2}\right) .
$$

Final remarks. This paper has been greatly influenced by work of Seidel [Sd1] and Piunikhin-Salamon-Schwartz [PSS]. As in [PSS], it is possible to define operations analogous to $\Psi_{\phi, \sigma}$ on fibrations over other compact Riemann surfaces besides the sphere. Our approach to Gromov-Witten invariants could also be adapted to deal with general symplectic fibrations $X \rightarrow B$ with arbitrary (non symplectic) base manifold $B$. This would lead to generalizations of the fiberwise (or family) Gromov-Witten invariants that were considered in the semi-positive case by Le-Ono in [LO], and would allow one to apply Seidel's ideas in [Sd2] to any symplectic manifold: cf. Remark 3.14.

Acknowledgements The author has profited from many conversations about this details of this paper with Polterovich and Lalonde. Any remaining mistakes are her own responsibility. She also wishes to thank Seidel for stimulating conversations, Salamon for suggesting that the virtual moduli cycle is a branched manifold, Siebert, Fukaya and Tian for useful discussions about general Gromov-Witten invariants, and ETH, Zurich, for its hospitality during part of the work on this paper.

\section{Proof of Theorem 1.1}

In this section we define the operation $\Psi_{\phi, \sigma}$ on quantum homology and discuss its main properties. Since these are based on facts about Gromov-Witten invariants, $\S 2.2$ is devoted to a preliminary discussion of these. In $\S 2.3$ we prove the basic propositions 2.2 and 2.5 modulo Claim 2.11 and Lemma 2.12.

\subsection{Quantum operations}

We begin by defining the operation on quantum homology associated to $P_{\phi}$. We will start from a definition that looks a little different from that given in $\$ 1.3$ since our formulations there assumed the validity of Theorem 1.1. Two sections $\sigma, \sigma^{\prime}$ of $P_{\phi}$ are said to be equivalent if

$$
u_{\phi}(\sigma)=u_{\phi}\left(\sigma^{\prime}\right), \quad c_{\phi}(\sigma)=c_{\phi}\left(\sigma^{\prime}\right)
$$


Definition 2.1 Given a loop of Hamiltonian diffeomorphisms $\phi$ on $M$, and an equivalence class of sections $\sigma$ of $P_{\phi}$ with $d=2 c_{\phi}(\sigma)$, we define the $\Lambda$-linear map

$$
\Psi_{\phi, \sigma}: Q H_{*}(M) \rightarrow Q H_{*+d}(M)
$$

as follows. For $a \in H_{*}(M), \Psi_{\phi, \sigma}(a)$ is the class in $Q H_{*+d}(M)$ whose intersection with $b \in H_{*}(M)$ is given by:

$$
\Psi_{\phi, \sigma}(a) \cdot{ }_{M} b=\sum_{B \in \mathcal{H}} n_{P}(\iota(a), \iota(b) ; \sigma+\iota(B)) \otimes e^{-B} .
$$

Here $n_{P}\left(v, w ; \sigma^{\prime}\right)$ is the Gromov-Witten invariant which counts isolated $J$-holomorphic stable curves in $P_{\phi}$ of genus 0 and with two marked points, that represent the equivalence class $\sigma^{\prime}$ and whose marked points go through given generic representatives of the classes $v$ and $w$ in $H_{*}\left(P_{\phi}\right) \cdot 6$ Note that, by Gromov compactness, there are for each given energy level $\kappa$ only finitely many section classes $\sigma^{\prime}=\sigma+\iota(B)$ with $\omega(B) \leq \kappa$ that are represented by $J$-holomorphic curves in $P_{\phi}$. Thus $\Psi_{\phi, \sigma}(a)$ satisfies the finiteness condition for elements of $Q H_{*}(M, \Lambda)$.

For reasons of dimension, $n_{P}\left(v, w ; \sigma^{\prime}\right)=0$ unless

$$
2 c_{\phi}\left(\sigma^{\prime}\right)+\operatorname{dim}(v)+\operatorname{dim}(w)=2 n .
$$

(Recall from $\S 1.2$ that $c_{\phi}$ is the vertical Chern class, not the full first Chern class $c_{1}$ of $P$. Thus $c_{\phi}\left(\sigma^{\prime}\right)=c_{1}\left(\sigma^{\prime}\right)-2$.) Further

$$
\Psi_{\phi, \sigma}(a)=\sum a_{\sigma, B} \otimes e^{-B}, \quad a_{\sigma, B} \in H_{*}(M),
$$

where $a_{\sigma, B} \cdot M b=n_{P}(\iota(a), \iota(b) ; \sigma+\iota(B))$, and

$$
\operatorname{dim}\left(a_{\sigma, B}\right)=\operatorname{dim}(a)+2 c_{\phi}(\sigma+\iota(B))=\operatorname{dim}(a)+2 c_{\phi}(\sigma)+2 c(B) .
$$

Observe also that

$$
\Psi_{\phi, \sigma+A}=\Psi_{\phi, \sigma} \otimes e^{A} .
$$

The rest of this section is concerned with two basic propositions. Here is the first.

Proposition 2.2 If $\phi$ is the constant loop $*$ and $\sigma_{0}$ is the class of the flat section $\left[p t \times S^{2}\right]$ in $P_{*}=M \times S^{2}$, then $\Psi_{*, \sigma_{0}}$ is the identity map.

The second deals with the behavior of $\Psi_{\sigma, \phi}$ under composition of loops, and before stating it we need some preparation. One can represent the composite $\psi * \phi$ either by the product $\left\{\psi_{t} \circ \phi_{t}\right\}$ or by the concatenation of loops.

Lemma 2.3 The bundle $P_{\psi * \phi}$ is diffeomorphic to the fiber sum $P_{\psi} \#_{M} P_{\phi}$.

\footnotetext{
${ }^{6}$ Observe that $n_{P}\left(v, w ; \sigma^{\prime}\right)$ is identical to the 3-point invariant $n_{P}\left(v, w,[M] ; \sigma^{\prime}\right)$ and we will use these notations interchangeably. See equation $(\emptyset$ in $\S 2.2 .2$.
} 
Proof: Recall that

$$
P_{\phi}=\left(D_{+}^{2} \times M\right) \cup_{\phi}\left(D_{-}^{2} \times M\right),
$$

where

$$
\phi_{t}:(2 \pi t, x)_{+} \mapsto\left(-2 \pi t, \phi_{t}(x)\right)_{-} .
$$

Let $M_{\phi, \infty}$ denote the fiber at $0 \in D_{2}^{-}$in $P_{\phi}$ and $M_{\psi, 0}$ the fiber at $0 \in D_{2}^{+}$in $P_{\psi}$. Cut out open product neighborhoods of each of these fibers and then glue the complements by an orientation reversing symplectomorphism of the boundary. The resulting space may be realised as

$$
\left(D_{2}^{+} \times M\right) \cup_{\alpha_{\phi,-1}}\left(S^{1} \times[-1,1] \times M\right) \cup_{\alpha_{\psi, 1}}\left(D_{2}^{-} \times M\right),
$$

where

$$
\alpha_{\phi,-1}(2 \pi t, x)=\left(2 \pi t,-1, \phi_{t}(x)\right), \quad \alpha_{\psi, 1}(2 \pi t, 1, x)=\left(-2 \pi t, \psi_{t}(x)\right),
$$

and this may clearly be identified with $P_{\psi * \phi}$.

Using the above notation, let

$$
\begin{aligned}
& V_{\phi}=\left(D_{2}^{+} \times M\right) \cup_{\alpha_{\phi,-1}}\left(S^{1} \times[-1,1 / 2) \times M\right), \\
& V_{\psi}=\left(S^{1} \times(-1 / 2,1] \times M\right) \cup_{\alpha_{\psi, 1}}\left(D_{2}^{-} \times M\right)
\end{aligned}
$$

The following lemma was proved in [LMP2].

Lemma 2.4 The classes $u_{\psi * \phi}$ and $c_{\psi * \phi}$ are compatible with the decomposition $P_{\psi * \phi}=$ $V_{\psi} \cup V_{\phi}$ in the sense that their restrictions to $V_{\psi} \cap V_{\phi}=(-1 / 2,1 / 2) \times S^{1} \times M$ equal the pullbacks of $[\omega]$ and $c_{1}(T M)$ under the obvious projection $V_{\psi} \cap V_{\phi} \rightarrow M$.

Given sections $\sigma$ of $P_{\phi}$ and $\sigma^{\prime}$ of $P_{\psi}$ that agree over a small neighborhood of the fibers $M_{\phi, \infty}, M_{\psi, 0}$, we write $\sigma^{\prime} \# \sigma$ for the union of these sections in the fiber sum $P_{\psi} \# P_{\phi}=P_{\psi * \phi}$. Here is the second basic result.

Proposition 2.5 For any sections $\sigma$ of $P_{\phi}$ and $\sigma^{\prime}$ of $P_{\psi}$

$$
\Psi_{\psi, \sigma^{\prime}} \circ \Psi_{\phi, \sigma}=\Psi_{\psi * \phi, \sigma^{\prime} \# \sigma} .
$$

Corollary 2.6 $\Psi_{\phi, \sigma}$ is an isomorphism for all loops $\phi$ and sections $\sigma$.

Proof: Observe that there is a fiberwise diffeomorphism from $P_{\phi}$ to $P_{\phi^{-1}}$ that is a symplectomorphism on the fibers and covers an orientation reversing map on the bases. Given a section $\sigma$ of $P_{\phi}$, let $-\sigma$ denote the image of this section in $P_{\phi^{-1}}$. Then it is easy to check that $\sigma \#(-\sigma)$ is isotopic to the flat section in the trivial bundle $P_{\phi} \# P_{\phi^{-1}}=P_{*}$. Hence, by Propositions 2.2 and 2.5, the inverse of $\Psi_{\phi, \sigma}$ is $\Psi_{\phi^{-1},-\sigma}$.

Proof of Theorem 1.1:

The Wang sequence for the fibration $p: P_{\phi} \rightarrow S^{2}$

$$
\ldots \rightarrow H_{*}(M) \stackrel{\iota}{\rightarrow} H_{*}\left(P_{\phi}\right) \stackrel{\cap[M]}{\rightarrow} H_{*-2}(M) \rightarrow H_{*-1}(M) \stackrel{\iota}{\rightarrow} \ldots
$$


implies that the spectral sequence for $H_{*}\left(P_{\phi}\right)$ splits if and only if the map $\iota: H_{*}(M) \rightarrow$ $H_{*}\left(P_{\phi}\right)$ is injective. Now Gromov-Witten invariants are linear in each variable. Thus if $\iota(a)=0$ for some $a \neq 0$, then $\Psi_{\phi, \sigma}(a)=0$, a contradiction with the fact that $\Psi_{\phi, \sigma}$ is an isomorphism.

One can almost prove Propositions 2.2 and 2.5 using the axioms of general GromovWitten invariants. The missing step is to show that these invariants can be calculated in a set-up that is adapted to our fibered setting. In the rest of this section, we outline proofs of these propositions so that readers can see what is involved. Initially, we will use Siebert's approach to Gromov-Witten invariants since in some ways this is conceptually the simplest.

Example 2.7 Since Theorem 1.1 is already known in the case when the loop $\phi$ comes from a circle action, we give an example to show that not all loops in $\pi_{1}(\operatorname{Ham}(M))$ arise this way. Take $(M, \omega)=\left(S^{2} \times S^{2},(1+\lambda) \tau_{1} \oplus \tau_{2}\right)$, where $0<\lambda<1$ and $\tau_{i}$ is an area form on the $i$ th sphere with total area 1 . Put $G^{\lambda}=\operatorname{Ham}(M, \omega)$. Given loops $u_{t}, v_{t}, t \in S^{1}$, in $G^{\lambda}$, let $u \times v$ denote the map

$$
u \times v: T^{2} \rightarrow G^{\lambda}: \quad(s, t) \mapsto u_{s} v_{t} .
$$

First observe that, if $h_{t}, t \in S^{1}$, is homotopic to a circle action then $h \times h$ is nullhomotopic. Indeed, we can assume that $h_{s} h_{t}=h_{s+t}$ so that the map $h \times h$ extends to $S^{1} \times D^{2}$ where $\partial D_{2}$ is attached to the circles $s+t=$ const. Now think of $S^{2} \times S^{2}$ as a fibration over the first sphere and let $\sigma_{S}, \sigma_{N}$ be two sections of the form $S^{2} \times\{p t\}, \sigma_{-}$be the anti-diagonal and $\sigma_{+}$the diagonal. Further, for $t \in S^{1}$, let $u_{t}$, (resp. $v_{t}$,) be a full rotation in the fibers that fixes the sections $\sigma_{N}, \sigma_{S}$ (resp. $\sigma_{ \pm}$.) Because $\lambda>0$ we can represent $u_{t}, v_{t}$ by loops in $G^{\lambda}$. Moreover, it follows from Anjos's calculation of the Pontriagin ring $H_{*}\left(G^{\lambda}, \mathbf{Z} / 2 \mathbf{Z}\right)$ in [An] that

$$
[u \times v] \neq[v \times u] \in H_{2}\left(G^{\lambda}, \mathbf{Z} / 2 \mathbf{Z}\right) .
$$

Thus $(u+v) \times(u+v)=u \times v+v \times u \neq 0$, so that the element $u+v$ is not homotopic to a circle action.

\subsection{Gromov-Witten invariants}

For the convenience of the reader we begin by recalling the definition of stable maps. For further information, see any of the papers $[\mathrm{FO}],[\mathrm{LiT}],[\mathrm{LiuT}],[\mathrm{R}]$ or $[\mathrm{S}]$. Useful background may be found in [MS1] or [RT].

\subsubsection{Stable maps and curves}

Given a symplectic manifold $(P, \omega)$ with compatible almost complex structure $J$, denote by $\mathcal{M}_{0, k}(P, J, A)$ the space of (unparametrized) $J$-holomorphic spheres in class $A$ with $k$ marked points. Elements of this space are equivalence classes $\left[h, z_{1}, \ldots, z_{k}\right]$ of elements $\left(h, z_{1}, \ldots, z_{k}\right)$, where $h: S^{2} \rightarrow P$ is a somewhere injective (i.e. nonmultiply covered) $J$-holomorphic map and the $z_{i}$ are distinct points in $S^{2}$. Elements $\left(h, z_{1}, \ldots, z_{k}\right)$ and $\left(h^{\prime}, z_{1}^{\prime}, \ldots, z_{k}^{\prime}\right)$ are equivalent if there is $\gamma \in \operatorname{PSL}(2, \mathbf{C})$ such that $h^{\prime}=h \circ \gamma$, and $z_{j}=\gamma\left(z_{j}^{\prime}\right)$ for all $j$.

When $J$ is generic, $\mathcal{M}_{0, k}(P, J, A)$ is a manifold of dimension

$$
\operatorname{dim}(P)+2 c_{1}(T P)(A)+2 k-6=2 n+4+2 c_{\phi}(A),
$$


when $\operatorname{dim} P=2 n+2, k=2$ and $A$ is a section class of $P=P_{\phi}$. However it is usually not compact. Its compactification $\overline{\mathcal{M}}_{0, k}(P, J, A)$ consists of $J$-holomorphic stable maps, i.e. of equivalence classes $\tau=\left[\Sigma, h, z_{1}, \ldots, z_{k}\right]$ of elements $\left(\Sigma, h, z_{1}, \ldots, z_{k}\right)$. Here the domain $\Sigma$ is a connected union of components $\Sigma_{i}, i=0, \ldots, \ell-1$, each of which has a given identification with $S^{2}$. (Note that we consider $\Sigma$ to be a topological space: the labelling of its components is a convenience and not part of the data.) The intersection pattern of the components can be described by a tree graph with $\ell$ vertices, where each edge corresponds to an intersection point of the components corresponding to its vertices. No more than two components meet at any point. There are also $k$ marked points $z_{1}, \ldots, z_{k}$ placed anywhere on $\Sigma$ except at an intersection point of two components. The restriction $h_{i}$ of the map $h$ to $\Sigma_{i}$ is assumed to be $J$-holomorphic and we impose the stability condition that $h_{i}$ is nonconstant (though possibly a multiple covering) unless $\Sigma_{i}$ contains at least 3 special points. (By definition, special points are either points of intersection with other components or marked points.) Also $h_{*}([\Sigma])=\sum_{i}\left(h_{i}\right)_{*}\left[\Sigma_{i}\right]=A$. Finally, we divide out by all holomorphic reparametrizations. In other words if $\gamma: \Sigma \rightarrow \Sigma$ is a holomorphic map such that $\gamma\left(z_{j}^{\prime}\right)=z_{j}$ for all $j$ we consider $\left(\Sigma, h \circ \gamma, z_{1}^{\prime}, \ldots, z_{k}^{\prime}\right)$ and $\left(\Sigma, h, z_{1}, \ldots, z_{k}\right)$ to be equivalent. Note that when $k=2$ the group of such reparametrizations for which $z_{j}^{\prime}=z_{j}$ for all $j$ has (real) dimension at least 2. (The other reparametrizations in some sense do not count since they can be gotten rid of by normalizing the choice of $z_{j}$.)

This defines the elements $\tau$ of $\overline{\mathcal{M}}=\overline{\mathcal{M}}_{0, k}(P, J, A)$. We write $e v$ for the evaluation map

$$
e v: \overline{\mathcal{M}}_{0, k}(P, A, J) \rightarrow P^{k}: \quad\left[\Sigma, h, z_{1}, \ldots, z_{k}\right] \rightarrow\left(h\left(z_{1}\right), \ldots, h\left(z_{k}\right)\right) .
$$

Each such $\tau$ has a finite automorphism group $\Gamma_{\tau}=\operatorname{Aut}\left(\left[\Sigma, h, z_{1}, \ldots, z_{k}\right]\right)$. Given a representative $\left(\Sigma, h, z_{1}, \ldots, z_{k}\right)$ of $\tau$ we may identify $\Gamma_{\tau}$ with the group of all holomorphic maps $\gamma: \Sigma \rightarrow \Sigma$ such that $h \circ \gamma=h$, and $\gamma\left(z_{j}\right)=z_{j}$ for all $j$. For most elements this group is trivial, and it is always finite because of the stability condition. It may be nontrivial if any of the $h_{i}$ are multiple coverings, or if two different $h_{i}$ have the same image. (Note that $\gamma$ might permute the components of $\Sigma$.) It is the presence of these automorphism groups that makes it impossible to find a manifold that regularizes $\overline{\mathcal{M}}=\overline{\mathcal{M}}_{0, k}(P, J, A)$.

The space of all stable maps has a natural topology in which $\overline{\mathcal{M}}$ is compact. We will later consider a small neighborhood $\mathcal{W}$ of $\overline{\mathcal{M}}$ in this space. $\mathcal{W}$ has a natural coarse stratification in which each stratum consists of elements $\tau=\left[\Sigma_{\tau}, h_{\tau}, z_{1}, \ldots, z_{k}\right]$ whose domain $\Sigma_{\tau}=\cup_{i} \Sigma_{i}$ has fixed topological type, where the marked points $z_{1}, \ldots, z_{k}$ are on specified components of $\Sigma$, and where the decomposition $A=\sum A_{i}$ is given. It also has a fine stratification in which we add the requirement that the isomorphism class of $\Gamma_{\tau}$ is fixed on each stratum. Each such stratum in $\mathcal{W}$ has a smooth topology modelled on the space of smooth (i.e. in some Sobolev class $L^{k, p}$ ) sections of a suitable bundle over the (fixed) domain $\Sigma$. However, this smooth structure is not extended over $\mathcal{W}$ as a whole: see $\S 4$. It is not hard to check that the index of the linearization $D h_{\tau}$ of the Cauchy-Riemann operator at $h_{\tau}$ depends only on the number $\ell$ of components of the domain $\Sigma_{\tau}$ and equals

$$
\text { ind } h_{\tau}=\operatorname{dim} \mathcal{M}-2(\ell-1)=2 n+2 c_{\phi}(A)+2 k+2-2 \ell,
$$

where the second equality holds when $P=P_{\phi}$ and $A$ is a section class.

There is a special case in which $P$ is a single point. In this case all maps are trivial, and the resulting space $\overline{\mathcal{M}}_{0, k}(p t, J, A)$ is usually written $\overline{\mathcal{M}}_{0, k}$. Its elements are called 
stable curves. The stability condition now says that every (nondegenerate) component of $\Sigma$ contains at least 3 special points. When $k<3$ the space $\overline{\mathcal{M}}_{0, k}$ reduces to a single point, that for consistency is taken to be represented by the degenerate Riemann surface consisting of a single point. The space $\overline{\mathcal{M}}_{0, k}$ also is a single point, but now this is represented by a sphere with 3 marked points. There is a forgetful map

$$
f: \overline{\mathcal{M}}_{0, k}(P, J, A) \rightarrow \overline{\mathcal{M}}_{0, k}: \quad\left[\Sigma, h, z_{1}, \ldots, z_{k}\right] \mapsto\left[\Sigma^{\prime}, z_{1}, \ldots, z_{k}\right],
$$

where $\left[\Sigma^{\prime}, z_{1}, \ldots, z_{k}\right]$ is the stabilization of the domain $\left[\Sigma, z_{1}, \ldots, z_{k}\right]$ of $h$ that is obtained by collapsing to a point each component of $\Sigma$ that does not contain at least 3 special points. (The domain $\left[\Sigma, z_{1}, \ldots, z_{k}\right]$ is called a prestable curve.) Note that, for $k \geq 3, \overline{\mathcal{M}}_{0, k}$ is a smooth oriented compact manifold of dimension $2 k-6$ : see [HS2] for example.

We now discuss stable maps in the context of our fibered space $P \rightarrow S^{2}$. As always, $\Omega$ is a compatible symplectic form on $P$.

Definition 2.8 We say that an almost complex structure $J$ on $P$ is fibered if the following conditions hold. At each point $x \in P$ let $H_{x}$ denote the $\Omega$-orthogonal to the tangent space to the fiber. Then $J$ must preserve the splitting $T_{x} P_{\phi}=T_{x}$ (Fiber) $\oplus H_{x}$, be $\Omega$-compatible and be such that the projection $p$ is $(J, j)$-holomorphic for the standard complex structure $j$ on $S^{2}$.

The next lemma explains the structure of $J$-holomorphic stable maps with two marked points when $J$ is fibered.

Lemma 2.9 When $P=P_{\phi}, J$ is fibered and $\sigma$ is a section class, each element $\left[\Sigma, h, z_{0}, z_{1}\right]$ of $\overline{\mathcal{M}}_{0,2}(P, J, \sigma)$ contains one component $\left(\Sigma_{0}, h_{0}\right)$, called the stem, that is a section of $p$ : $P \rightarrow S^{2}$. All other components lie in the fibers of $P$ and may be divided into a finite number of connected pieces called branches, each branch lying in a different fiber.

Proof: When $J$ is fibered, any $J$-holomorphic curve $h: S^{2} \rightarrow P$ that represents the class of a section must intersect each fiber of $P$ exactly once transversally since $p \circ h: S^{2} \rightarrow S^{2}$ is a holomorphic map of degree 1 . Further, any $J$-holomorphic curve $h: S^{2} \rightarrow P$ that represents a class $B$ such that $B \cdot[M]=0$ must lie entirely in a fiber, and no class with $B \cdot[M]<0$ has a $J$-holomorphic representative. Therefore, given a $J$-holomorphic stable map $[\Sigma, h]$ in the class $\sigma$, in the corresponding decomposition $\sigma=\sum B_{i}$ of $\sigma$ (where $B_{i}=h_{*}\left(\Sigma_{i}\right)$ ) we must have $B_{i} \cdot[M] \geq 0$ for all $i$. It follows that $B_{i} \cdot[M]=0$ for all $i$ except one (that we choose to be $i=0$ ). Each other component lies entirely in a fiber. Moreover the union of the components in a given fiber must be connected since $h(\Sigma)$ is connected and the stem $h\left(\Sigma_{0}\right)$ intersects each fiber just once. The result is now immediate.

Each branch can be considered as an element of some moduli space $\overline{\mathcal{M}}_{0, k}\left(M, J_{M}, B\right)$, where $1 \leq k \leq 3$. For each branch contains one marked point where it meets the stem and may or may not also contain $z_{1}$ or $z_{2}$.

\subsubsection{Gromov-Witten invariants}

Siebert's point of view is the following. In order to count the number of isolated $J$ holomorphic $A$-curves of genus 0 in $(P, J)$ through the $k$ classes $\alpha_{1}, \ldots, \alpha_{k} \in H_{*}(P)$, one 
considers the space $\overline{\mathcal{M}}_{0, k}(P, A, J)$ of $J$-holomorphic genus 0 stable maps in class $A$ with $k$ marked points. This space supports a virtual fundamental class

$$
\mathcal{C}_{A, k}^{h o l}(P, J) \in H_{d}^{B M}\left(\overline{\mathcal{M}}_{0, k}(P, A, J), \mathbf{Q}\right)
$$

of dimension $d=\operatorname{dim} P+2 c_{1}(A)+2 k-6$, where $H^{B M}$ denotes the Borel-Moore homology. In the nicest cases, $\overline{\mathcal{M}}_{0, k}(P, A, J)$ is a compact manifold of dimension $d$ and $\mathcal{C}_{A, k}^{\text {hol }}(P, J)$ is simply its fundamental class. More generally, (e.g. in the semi-positive case, see [MS1]) $\overline{\mathcal{M}}_{0, k}(P, A, J)$ is a compact stratified space whose top stratum is a manifold of dimension $d$ and all other strata have dimension $\leq d-2$. In this case too $\overline{\mathcal{M}}_{0, k}(P, A, J)$ carries a fundamental class and $\mathcal{C}_{A, k}^{\text {hol }}(P, J)=\overline{\mathcal{M}}_{0, k}(P, A, J)$.

Given $k$ homology classes $v_{1}, \ldots, v_{k}$ in $P$, the Gromov-Witten invariant $n_{P}\left(v_{1}, \ldots, v_{k} ; A\right)$ is defined to be the intersection number

$$
n_{P}\left(v_{1}, \ldots, v_{k} ; A\right)=e v\left(\mathcal{C}_{A, k}^{h o l}(P, J)\right) \cdot P^{k}\left(v_{1} \times \ldots \times v_{k}\right)
$$

of the pushforward $e v_{*}\left(\mathcal{C}_{A, k}^{\text {hol }}(P, J)\right)$ of $\mathcal{C}_{A, k}^{\text {hol }}(P, J)$ by the evaluation map with the class $v_{1} \times$ $\ldots \times v_{k}$ in $H_{*}\left(P^{k}, \mathbf{Q}\right)$. By definition, this number is zero unless the dimensions of the cycles are complementary in $P^{k}$. Thus if $P=P_{\phi}$ and $k=2$ we need

$$
d+\operatorname{dim} v_{1}+\operatorname{dim} v_{2}=2(2 n+2),
$$

which reduces to the dimension condition $2 c_{\phi}(A)+\operatorname{dim} v_{1}+\operatorname{dim} v_{2}=2 n$ in equation (3) above. Note also that $n_{P}\left(v_{1}, \ldots, v_{k} ; A\right)=0$ if $A \neq 0$ and any $v_{i}=[P]$ since in that case it is impossible for the intersections to be isolated.

The invariant $n_{P}\left(v_{1}, \ldots, v_{k} ; A\right)$ is the most simple-minded Gromov-Witten invariant. More generally, one can consider the forgetful map

$$
f: \overline{\mathcal{M}}_{0, k}(P, A, J) \rightarrow \overline{\mathcal{M}}_{0, k},
$$

take a class $c \in H_{*}\left(\overline{\mathcal{M}}_{0, k}, \mathbf{Q}\right)$, and then set

$$
n_{P, c}\left(v_{1}, \ldots, v_{k} ; A\right)=(f \times e v)_{*}(\mathcal{C}) \cdot\left(c \times v_{1} \times \ldots \times v_{k}\right),
$$

where the intersection is taken in $\overline{\mathcal{M}}_{0, k} \times P^{k}$. Thus our previous definition corresponds to taking $c$ to be the fundamental class of $\overline{\mathcal{M}}_{0, k}$.

When $k \leq 3, \overline{\mathcal{M}}_{0, k}$ is a single point, and so there is only one invariant. We also need to consider the case $k>3$. Let $\chi=[p t] \in H_{0}\left(\overline{\mathcal{M}}_{0, k}\right)$. The invariant

$$
n_{P, \chi}\left(v_{1}, \ldots, v_{k} ; A\right)
$$

intuitively speaking counts the number of $J$-holomorphic maps $h: S^{2} \rightarrow P$ such that $h\left(z_{i}\right) \in v_{i}$ for a fixed set of points $z_{1}, \ldots, z_{k}$. In particular, if $k=4$ fixing the points $z_{i}$ is equivalent to fixing their cross ratio. Hence $n_{P, \chi}\left(v_{1}, \ldots, v_{4} ; A\right)$ is calculated using the class $\mathcal{C}_{A, 4}^{\text {hol }}(P, J) \cap\left[\overline{\mathcal{M}}_{t}\right]$ in $H_{*}\left(\overline{\mathcal{M}}_{t}\right)$, where $\overline{\mathcal{M}}_{t}$ is the subset of $\overline{\mathcal{M}}=\overline{\mathcal{M}}_{0,4}(P, J, A)$ on which the cross ratio of the marked points is fixed at $t$.

\footnotetext{
7 The cross ratio on $\overline{\mathcal{M}}_{0,4}(P, J, A)$ can be identified with the forgetful map to the space $\overline{\mathcal{M}}_{0,4}=S^{2}$ of genus zero stable curves with 4 marked points. For generalizations of this, see Hofer-Salamon [HS2].
} 
An important identity, that is equivalent to the associativity of quantum multiplication, is the following:

$$
n_{P, \chi}\left(v_{1}, \ldots, v_{4} ; A\right)=\sum_{A=A_{1}+A_{2}, \alpha} n_{P}\left(v_{1}, v_{2}, e_{\alpha}^{\prime} ; A_{1}\right) \cdot n_{P}\left(f_{\alpha}^{\prime}, v_{3}, v_{4} ; A_{2}\right)
$$

where $\left\{e_{\alpha}^{\prime}\right\}_{\alpha}$ is a basis for the homology $H_{*}(P, \mathbf{Q})$, and $\left\{f_{\beta}^{\prime}\right\}_{\beta}$ is the dual basis with respect to the intersection pairing. Further, if $v_{4}=[P]$, we have

$$
n_{P, \chi}\left(v_{1}, v_{2}, v_{3},[P] ; A\right)=n_{P}\left(v_{1}, v_{2}, v_{3} ; A\right) .
$$

(This is consistent with (6) since in this case the only term that contributes to the sum has $A_{2}=0$.) Another important point is that if $w \in H_{(\operatorname{dim} P)-2}(P)$ then

$$
n_{P}\left(v_{1}, v_{2}, w ; A\right)=(w \cdot A) n_{P}\left(v_{1}, v_{2} ; A\right) .
$$

In particular, if $P=P_{\phi}$ and $\sigma$ is a section class then

$$
n_{P}\left(v_{1}, v_{2},[M],[M] ; \sigma\right)=n_{P}\left(v_{1}, v_{2},[M] ; \sigma\right)=n_{P}\left(v_{1}, v_{2} ; \sigma\right) .
$$

Finally, we observe that part (i) of Proposition 1.6 follows immediately from the definitions. This states that $n_{P}(\iota(a), \iota(b), v ; \iota(A))$ is always 0 . To see this, observe that if $J$ is fibered Lemma 2.9 implies that every $J$-holomorphic stable map in class $\iota(A)$ has image in a single fiber. Hence if we represent the classes $\iota(a), \iota(b)$ by cycles in different fibers the evaluation map

$$
\text { ev }: \overline{\mathcal{M}}_{0,3}\left(P_{\phi}, J, \iota(A)\right) \rightarrow P_{\phi}^{3}
$$

never intersects the representative of $\iota(a) \times \iota(b) \times v$.

It is also easy to check that the rest of Proposition 1.6 holds when $P$ is so nice that there is a fibered $J$ such that $\overline{\mathcal{M}}_{0, k}(P, J, A)$ supports a fundamental class whenever $A$ is a section or fiber class. For then the relevant Gromov-Witten invariants can be calculated by simply counting $J$-holomorphic curves. 8

\subsection{Proofs of Propositions 2.2 and 2.5.}

In this section, we will prove these propositions modulo two statements Claim 2.11 and Lemma 2.12 about the behavior of Gromov-Witten invariants. To simplify our notation we will usually omit mention of the inclusion map $\iota: H_{*}(M) \rightarrow H_{*}(P)$, instead using the convention that $a, b, c, A, B$ denote elements of $H_{*}(M)$ and $v, w, \sigma$ denote elements of $H_{*}(P)$.

\subsubsection{Calculating $\Psi_{\phi, \sigma}$ for trivial bundles}

Proposition 2.2 says that $\Psi_{\phi, \sigma_{0}}$ is the identity when $\phi$ is the constant loop $*$ and $\sigma_{0}$ is the flat section. Thus we must show:

\footnotetext{
8 This is the case considered in [Sd1] and [LMP2]. To complete the argument in this case one must use some elementary transversality arguments that are similar to those in Lemma 4.14 below.
} 
Lemma 2.10 (i) $n_{P}\left(a, b ; \sigma_{0}+B\right)=0$ unless $B=0$,

(ii) $n_{P}\left(a, b ; \sigma_{0}\right)=a \cdot{ }_{M} b$.

\section{Proof of (ii).}

Let $\Omega$ be a product form on $P_{*}=M \times S^{2}, \alpha, \beta$ be cycles in different fibers of $P_{*}$ and $J$ be a product almost complex structure $J_{M} \times j$ on $P_{*}$. Then the projections $P_{*} \rightarrow M, P_{*} \rightarrow S^{2}$ are holomorphic, so that any $J$-holomorphic curve $\tilde{f}: S^{2} \rightarrow M \times S^{2}$ in class $\sigma_{0}$ projects to a constant in $M$. Thus, the space $\mathcal{M}_{0,2}\left(P, \sigma_{0}, J\right)$ of unparametrized $J$-holomorphic spheres in class $\sigma_{0}$ is a compact manifold that can be identified with $M$. In this case the virtual fundamental class $\mathcal{C}_{A, k}^{\text {hol }}(P, J)$ is simply the fundamental class of $\mathcal{M}_{0,2}\left(P_{*}, \sigma_{0}, J\right)$. It follows immediately that $n_{P}\left(a, b ; \sigma_{0}\right)=a \cdot M b$.

\section{Sketch Proof of (i).}

Now consider calculating $n_{P}\left(a, b ; \sigma_{0}+B\right)$ where $B \neq 0$. First observe that when $J=$ $J_{M} \times j$ there is a well defined map

$$
g r: \overline{\mathcal{M}}_{0,3}\left(M, B, J_{M}\right) \rightarrow \overline{\mathcal{M}}_{0,2}\left(M \times S^{2}, \sigma_{0}+B, J\right)
$$

given on $\tau=\left[\Sigma_{\tau}, h, z_{0}, z_{1}, z_{2}\right]$ as follows. If the domain $\Sigma_{\tau}$ has just one component, then there is a unique map $\gamma: \Sigma_{\tau} \rightarrow S^{2}$ that takes the 3 marked points to $q_{0}=0, q_{1}=1, q_{2}=\infty$. Then set $\operatorname{gr}(\tau)=\left[\Sigma_{\tau}, \widehat{h}, z_{0}, z_{1}\right]$ where

$$
\widehat{h}(z)=(h(z), \gamma(z)) \in M \times S^{2} .
$$

Thus, if we identify $\Sigma$ with $S^{2}$ via $\gamma, \widehat{h}$ is the graph of $h$.

In general, let us define the projection $p_{j}\left(z_{i}\right)$ of the marked point $z_{i}$ onto the component $\Sigma_{j}$ as follows. If $z_{i} \in \Sigma_{j}$ then $p_{j}\left(z_{i}\right)=z_{i}$. Otherwise it is the unique point on $\Sigma_{j}$ where the connected component of $\Sigma_{\tau}-\Sigma_{j}$ containing $z_{i}$ is attached to $\Sigma_{j}$. It is easy to see that for each domain $\left[\Sigma_{\tau}, z_{0}, z_{1}, z_{2}\right]$ there is precisely one component onto which the projections of the three marked points are distinct. Let us call this component $\Sigma_{0}$. It contains three distinct points $p_{0}\left(z_{i}\right)$ and so we can define $\widehat{h}$ on this component as before. This is the stem of $\operatorname{gr}(\tau)$. The other components are lifted as branch components in the obvious way.

It is not hard to check that the map $g r$ is continuous. Moreover, its image is precisely the set of elements of $\overline{\mathcal{M}}_{0,2}\left(M \times S^{2}, \sigma_{0}+B, J\right)$ that take $z_{i}$ into $M \times q_{i}$ for $i=0,1$. Hence, $g r$ is essentially surjective. (More precisely, its image contains all stable maps, but the marked points might be in the wrong place.) On the other hand, when we calculate the GromovWitten invariant $n_{P}\left(a, b ; \sigma_{0}+B\right)$ it is all right to have the marked points in designated fibers. Indeed, let us consider the evaluation map

$$
e v_{01}: \operatorname{Im} g r \rightarrow M \times M,
$$

where the range $M \times M$ is identified with $\left(M \times\left\{q_{0}\right\}\right) \times\left(M \times\left\{q_{1}\right\}\right)$ in $P \times P$. Observe that the formal codimension of its image is the same as that of the map

$$
\text { ev }: \overline{\mathcal{M}}_{0,2}\left(M \times S^{2}, \sigma_{0}+B, J\right) \rightarrow P \times P .
$$

We will prove an equivalent of the following claim in Lemma 4.15. 
Claim 2.11 If $\mathcal{C}$ is the virtual fundamental class of $\overline{\mathcal{M}}_{0,3}\left(M, B, J_{M}\right)$, then the invariant $n_{P}\left(a, b ; \sigma_{0}+B\right)$ is the intersection number of

$$
\left(e v_{01} \circ g r\right)_{*}(\mathcal{C})
$$

with the class $a \times b$ in $M \times M$

Now observe that the map $e v_{01} \circ g r$ reduces dimension: given

$$
\tau=\left[\Sigma, h, z_{0}, z_{1}, z_{2}\right] \in \overline{\mathcal{M}}_{0,3}\left(M, B, J_{M}\right),
$$

the element $\operatorname{gr}(\tau)$ varies as $z_{2}$ moves, but $e v_{01}(g r(\tau))$ remains unchanged. It follows easily that, for dimensional reasons, $a \times b$ can be perturbed in $M \times M$ to be disjoint from a representing cycle for the class $\left(e v_{01} \circ g r\right)_{*}(\mathcal{C})$. Hence $n_{P}\left(a, b ; \sigma_{0}+B\right)=0$.

\subsubsection{Proof of the composition rule}

It follows immediately from the definition of $\Psi$ (see Definition 2.1) and the remarks at the end of $\S 2.2 .2$ that, if $e_{i}$ is a basis for $H_{*}(M)$ with dual basis $\left\{f_{j}\right\}$, then

$$
\left.\Psi_{\phi, \sigma}(a)=\sum_{B, i} n_{P_{\phi}}\left(a,[M], e_{i}\right) ; \sigma+B\right) f_{i} \otimes e^{-B} .
$$

We would like to use equations (6) and (7) in $\S 2.2 .2$ to make the following calculation:

$$
\begin{aligned}
\Psi_{\psi, \sigma^{\prime}}\left(\Psi_{\phi, \sigma}(a)\right) & =\sum_{B, i} n_{P_{\phi}}\left(a,[M], e_{i} ; \sigma+B\right) \Psi_{\psi, \sigma^{\prime}}\left(f_{i}\right) \otimes e^{-B} \\
& =\sum_{B, B^{\prime}} \sum_{i, k} n_{P_{\phi}}\left(a,[M], e_{i} ; \sigma+B\right) \cdot \\
& =\sum_{A, k} n_{P_{\psi * \phi}, \chi}\left(a,[M],[M], f_{i},[M], e_{k} ; \sigma^{\prime}+B^{\prime}\right) f_{k} \otimes e^{-B-B^{\prime}} \\
& =\Psi_{\psi * \phi, \sigma \# \sigma^{\prime}}(a) .
\end{aligned}
$$

The only problem here is that we are considering Gromov-Witten invariants in different spaces. To overcome this difficulty we must define a space $\mathcal{X}$ that contains all the spaces $P_{\phi}, P_{\psi}$ and $P_{\psi * \phi}$. To this end, let $\pi:\left(\mathcal{S}, j_{\mathcal{S}}\right) \rightarrow \Delta$ be a singular holomorphic fibration over the unit disc in $\mathbf{C}$, with generic fiber $\Sigma_{t}=S^{2}$ and singular fiber $\Sigma_{0}$ over $\{0\}$ formed by the union of two transversally intersecting 2 -spheres. Thus there are local complex coordinates $(z, w)$ round the singular point in $\Sigma_{0}$ such that

$$
\pi(z, w)=z w .
$$

Observe that each component of $\Sigma_{0}$ has self-intersection -1 . Choose four disjoint smooth sections $s_{i}: \Delta \rightarrow \mathcal{S}$ such that $s_{1}(0), s_{2}(0)$ lie in one component of $\Sigma_{0}$ and $s_{3}(0), s_{4}(0)$ lie in the other, and so that the cross ratio of the four points $s_{i}(t)$ on $\Sigma_{t}$ uniquely determines $t$ when $t \neq 0$. This is a holomorphic model for the degeneration of the complex structure of 
the sphere $\Sigma_{t}$ with 4 marked points as a bubble is formed that contains two of the marked points.

We next construct a smooth fibration $q: \mathcal{X} \rightarrow \mathcal{S}$ with fiber $M$ such that

$$
X_{t}=\left.\mathcal{X}\right|_{\Sigma_{t}} \cong P_{\psi} \# P_{\phi}, \quad X_{0}=\left.\mathcal{X}\right|_{\Sigma_{0}}=P_{\phi} \cup P_{\psi},
$$

where in the union $P_{\phi} \cup P_{\psi}$ the fibers $M_{\psi, 0}$ are $M_{\phi, \infty}$ are identified. This can be done very explicitly using the description of $P_{\psi} \# P_{\phi}$ in $\S 1$. Moreover, we can suppose that $\mathcal{X}$ has a symplectic form $\widetilde{\Omega}$ and a compatible $\widetilde{J}$ that restrict appropriately on each $X_{t}$. (In particular, the projection $\mathcal{X} \rightarrow \mathcal{S}$ is holomorphic, which implies that $\widetilde{J}$ restricts to a fibered almost complex structure $J_{t}$ on each $X_{t}$.)

Now let $\widetilde{\alpha}_{i}, i=1,2,3,4$ be relative cycles in $(\mathcal{X}, \partial \mathcal{X})$ whose images by $q: \mathcal{X} \rightarrow \mathcal{S}$ equal the images of the sections $s_{i}: \Delta \rightarrow \mathcal{S}$ and are such that:

$$
\widetilde{\alpha}_{1} \cap X_{0}=\alpha, \quad \widetilde{\alpha}_{i}=q^{-1}\left(\operatorname{Im} s_{i}\right), i=2,3, \quad \widetilde{\alpha}_{4} \cap X_{0}=\beta,
$$

where $\alpha, \beta$ represent the classes $a, b$. Note that every $\widetilde{J}$-holomorphic stable map $\tau$ that meets these cycles $\widetilde{\alpha}_{i}$ must lie entirely in one of the sets $X_{t}$ (since the projection $\mathcal{X} \rightarrow \Delta$ is holomorphic). Moreover, the value of $t$ is determined by the cross ratio of the marked points on $\tau$.

Let $\widetilde{a}_{i} \in H_{*}(\mathcal{X}, \partial \mathcal{X})$ be the classes represented by the $\widetilde{\alpha}_{i}$. Then, even though $\mathcal{X}$ has a boundary, one should be able to make sense of Gromov-Witten invariants on $\mathcal{X}$. It follows easily from the calculation given above that Proposition 2.5 would hold if we could prove the following lemma.

Lemma 2.12 (i) If $\sigma$ is a section class in $X_{t}=P_{\psi * \phi}$ and $\widetilde{\sigma}$ is its image in $H_{2}(\mathcal{X})$, for each $t \neq 0$ we have:

$$
n_{\mathcal{X}, \chi}\left(\widetilde{a}_{1}, \ldots, \widetilde{a}_{4} ; \widetilde{\sigma}\right)=n_{X_{t}}(a,[M],[M], b ; \sigma) .
$$

(ii) The above statement also holds when $t=0$ if the right hand side is defined by:

$$
n_{X_{0}}(a,[M],[M], b ; \sigma)=\sum_{\sigma=\sigma_{1} \# \sigma_{2}, i} n_{P_{\phi}}\left(a,[M], e_{i} ; \sigma_{1}\right) n_{P_{\psi}}\left(f_{i},[M], b ; \sigma_{2}\right) .
$$

To justify (i) note that $n_{\mathcal{X}, \chi}$ is calculated by restricting attention to the part of the space $\overline{\mathcal{M}}_{0,4}(\mathcal{X}, \widetilde{J}, \widetilde{\sigma})$ on which the cross ratio is fixed, and, as pointed out above, the only elements in $\overline{\mathcal{M}}_{0,4}(\mathcal{X}, \widetilde{J}, \widetilde{\sigma})$ that meet the cycles $\widetilde{\alpha}_{i}$ lie in a single $X_{t}$.

A proof of this lemma will be given in $\S 4.3 .3$ below.

\section{Proofs of the other properties of $P_{\phi}$}

In this section we complete the proofs of the results announced in $\S 1.1$ and $\S 1.2$, assuming that Theorem 1.1 is proven and that Propositions 1.6, 2.2 and 2.5 hold. Proposition 1.6 itself is proved in $\S 4.3 .2$. 


\subsection{The homomorphism $\rho$.}

In order to use the maps $\Psi_{\phi, \sigma}$ to define $\rho$ we must make a canonical choice of section $\sigma_{\phi}$ that (up to equivalence) satisfies the composition rule

$$
\sigma_{\psi * \phi}=\sigma_{\phi} \# \sigma_{\psi},
$$

where $\sigma_{\phi} \# \sigma_{\psi}$ denotes the obvious union of the sections $\sigma_{\phi}, \sigma_{\psi}$ in $P_{\psi * \phi}=P_{\psi} \# P_{\phi}$. Unfortunately, it is not always possible to do this if one just considers ordinary sections. To get around this difficulty, we define the notion of a generalized section or $\mathbf{R}$-section.

Definition 3.1 We will say that $\sigma$ is an $\mathbf{R}$-section of $P_{\phi}$ if it is a finite $\operatorname{sum} \sum \lambda_{i} \sigma_{i}, \lambda_{i} \in \mathbf{R}$, of sections such that $p_{*}([\sigma])$ is the fundamental class of $S^{2}$. Two $\mathbf{R}$-sections $\sigma, \sigma^{\prime}$ are equivalent if

$$
u_{\phi}(\sigma)=u_{\phi}\left(\sigma^{\prime}\right), \quad c_{\phi}(\sigma)=c_{\phi}\left(\sigma^{\prime}\right) .
$$

The following lemma is immediate. (Part (iii) follows from Lemma 2.4.)

Lemma 3.2 (i) If the classes $[\omega]$ and $c_{1}(T M)$ are linearly independent on $H_{2}^{S}(M)$, there is for each $\phi$ a unique equivalence class of $\mathbf{R}$-sections $\sigma_{\phi}$ such that

$$
u_{\phi}\left(\sigma_{\phi}\right)=0, \quad c_{\phi}\left(\sigma_{\phi}\right)=0 .
$$

(ii) If the classes $[\omega]$ and $c_{1}(T M)$ are linearly dependent on $H_{2}^{S}(M)$ and $[\omega] \neq 0$, we choose $\sigma_{\phi}$ to be the unique equivalence class of $\mathbf{R}$-sections such that $u_{\phi}\left(\sigma_{\phi}\right)=0$. If $[\omega]=0$ on $H_{2}^{S}(M)$ but $c_{\phi} \neq 0$, we choose $\sigma_{\phi}$ so that $c_{\phi}\left(\sigma_{\phi}\right)=0$. If both $[\omega]$ and $c$ vanish on $H_{2}^{S}(M)$, then there is only one section class up to equivalence, and we take that for $\sigma_{\phi}$.

(iii) In all cases, $\sigma_{\psi * \phi}=\sigma_{\phi} \# \sigma_{\psi}$.

Remark 3.3 Note that it may happen that the classes $[\omega]$ and $c_{1}(T M)$ are linearly dependent on $H_{2}^{S}(M)$ while their extensions $u_{\phi}$ and $c_{\phi}$ are not linearly dependent on $H_{2}\left(P_{\phi}\right)$ : see Example 3.12. Therefore, there may be no $\mathbf{R}$-section such that $u_{\phi}\left(\sigma_{\phi}\right)=0, c_{\phi}\left(\sigma_{\phi}\right)=0$. This is why we used weaker conditions in (ii) above. Note however that when $\omega=0$ on $H_{2}^{S}(M)$ it follows from the proof of Proposition 3.23 in $\$ 3.4$ that the section $\sigma_{\phi}$ defined as above is integral and that $u_{\phi}\left(\sigma_{\phi}\right)=c_{\phi}\left(\sigma_{\phi}\right)=0$.

One should think of the $\mathbf{R}$-section $\sigma_{\phi}$ as an average of the sections in $P_{\phi}$. The effect of enlarging the Novikov ring to $\Lambda_{R}$ is thus to make enough room to take this average. More precisely, observe that the definition of the map $\Psi_{\phi, \sigma_{\phi}}$ still makes perfect sense provided that one allows the coefficients $B$ to belong to $\mathcal{H}_{R}=H_{2}^{S}(M, \mathbf{R}) / \sim$ so that the sum $\sigma_{\phi}+$ $\iota(B)$ can be integral. In the next proposition $\operatorname{Aut}_{\Lambda_{R}}\left(Q H_{*}\left(M, \Lambda_{R}\right)\right)$ denotes the group of automorphisms of the $\Lambda_{R}$-module $Q H_{*}\left(M, \Lambda_{R}\right)$.

Proposition 3.4 The map

$$
\rho^{\prime}: \pi_{1}(\operatorname{Ham}(M, \omega)) \rightarrow \operatorname{Aut}_{\Lambda_{R}}\left(Q H_{*}\left(M, \Lambda_{R}\right)\right)
$$

defined by $\rho^{\prime}(\phi)=\Psi_{\phi, \sigma_{\phi}}$ is a group homomorphism. 
Proof: This follows immediately from Propositions 2.2 and 2.5 and Lemma 3.2 .

Following Seidel, we now want to show that the automorphisms $\rho_{\phi}^{\prime}=\rho^{\prime}(\phi)$ respect the structure of $Q H_{*}(M)$ as a right $Q H_{*}(M)$-module, i.e.

$$
\rho_{\phi}^{\prime}\left(a *_{M} b\right)=\left(\rho_{\phi}^{\prime} a\right) *_{M} b .
$$

To this end, it is convenient to introduce the following more flexible notation for the quantum product. Let $\left\{e_{j}\right\}$ be a basis for $H_{*}(M)$ with dual basis $\left\{f_{j}\right\}$. Then, for any section $s^{\prime}: H_{*}(M) \rightarrow H_{*+2}(P)$

$$
\iota\left(e_{i}\right) \cdot P s^{\prime}\left(f_{j}\right)=e_{i} \cdot M f_{j}=s^{\prime}\left(e_{i}\right) \cdot P \iota\left(f_{j}\right)=\delta_{i j} .
$$

The following lemma shows that we can modify $s^{\prime}$ to a section $s$ such that in addition $s\left(e_{i}\right) \cdot s\left(e_{j}\right)=s\left(f_{i}\right) \cdot s\left(f_{j}\right)=0$ for all $i, j$. To simplify our notation we will from now on omit mention of the inclusion $\iota: H_{*}(M) \rightarrow H_{*}(P)$, but will keep the convention that the letters $a, b, c, e, f$ refer to homology classes on $M$ and $u, v, w$ to those in $P$.

Lemma 3.5 In the above situation, let $d(e)$ denote the dimension of the class $e \in H_{*}(M)$, let $2 n=\operatorname{dim}(M)$, set $q_{i j}=s^{\prime}\left(e_{i}\right) \cdot s^{\prime}\left(e_{j}\right)$, and define

$$
s\left(e_{i}\right)=\left\{\begin{array}{lll}
s^{\prime}\left(e_{i}\right) & \text { if } & d\left(e_{i}\right)<n-1 \\
s^{\prime}\left(e_{i}\right)-\frac{1}{2} \sum_{j}(-1)^{n-1} q_{i j} f_{j} & \text { if } & d\left(e_{i}\right)=n-1 \\
s^{\prime}\left(e_{i}\right)-\sum_{j}(-1)^{d\left(e_{j}\right)} q_{i j} f_{j} & \text { if } & d\left(e_{i}\right)>n-1,
\end{array}\right.
$$

where the sums are over those $j$ for which $f_{j}$ has the appropriate dimension, i.e. $d\left(f_{j}\right)+$ $d\left(e_{j}\right)=2 n$. Then, if the $s\left(f_{j}\right)$ are defined by linearity,

$$
e_{i} \cdot P s\left(f_{j}\right)=\delta_{i j}, \quad s\left(e_{i}\right) \cdot P s\left(f_{j}\right)=0
$$

for all $i, j$.

Proof: This is an easy calculation that is left to the reader.

From now on we will assume that the $e_{j}, f_{j}$ and $s$ are as above. Then the elements $\left\{e_{j}, s\left(e_{k}\right)\right\}$ form a basis for $H_{*}(P)$ whose dual basis is given by $\left\{s\left(f_{j}\right), f_{k}\right\}$. Similarly, the basis $\left\{f_{j}, s\left(e_{k}\right)\right\}$ has dual basis

$$
\left\{(-1)^{\varepsilon_{j}} s\left(e_{j}\right), f_{k}\right\}, \quad \text { where } \varepsilon_{j} \equiv d\left(e_{j}\right) \quad(\bmod 2) .
$$

Observe that in our present notation

$$
\begin{aligned}
a *_{M} b & =\sum_{j, B \in \mathcal{H}_{R}} n_{M}\left(a, b, e_{j} ; B\right) f_{j} \otimes e^{-B} \in Q H_{*}(M, \Lambda), \\
\Psi_{\phi, \sigma}(a) & =\sum_{j, B \in \mathcal{H}_{R}} n_{P}\left(a,[M], e_{j} ; \sigma+B\right) f_{j} \otimes e^{-B} .
\end{aligned}
$$

It follows easily that the triple invariants $n_{P}(a, b, c ; \sigma)$ are the coefficients of $\Psi_{\phi, \sigma}(a) *_{M} b$. Thus, we have: 


\section{Corollary 3.6}

$$
\Psi_{\phi, \sigma}(a) *_{M} b=\sum_{B, j} n_{P}\left(a, b, e_{j} ; \sigma+B\right) f_{j} \otimes e^{-B} .
$$

Similarly, the horizontal quantum product $*_{H, \sigma}$ that was introduced in $\S 1.3$ may be written as:

$$
\begin{aligned}
& u *_{H, \sigma} v=\sum_{j, B \in \mathcal{H}_{R}} n_{P}\left(u, v, e_{j} ; \sigma+B\right) s\left(f_{j}\right) \otimes e^{-B} \\
&+n_{P}\left(u, v, s\left(e_{j}\right) ; \sigma+B\right) f_{j} \otimes e^{-B} .
\end{aligned}
$$

Proposition 3.7 (i) $\Psi_{\phi, \sigma}(a)=\left([M] *_{H, \sigma} a\right) \cap[M]$.

(ii) Let $Q_{\sigma}=\Psi_{\phi, \sigma}([M])=\left([M] *_{H, \sigma}[M]\right) \cap[M]$. Then

$$
\Psi_{\phi, \sigma}(a)=Q_{\sigma} *_{M} a .
$$

Proof: The first statement follows immediately from Definition 2.1 since $s\left(f_{j}\right) \cap[M]=$ $f_{j}, f_{j} \cap[M]=0$. To prove (ii) observe first that

$$
\begin{aligned}
\Psi_{\phi, \sigma}(a) & =\left([M] *_{H, \sigma} a\right) \cap[M] \\
& =\sum_{j, B \in \mathcal{H}_{R}} n_{P}\left([M], a, e_{j} ; \sigma+B\right) f_{j} \otimes e^{-B} \\
& =\sum_{B, A, k, j} n_{P}\left([M],[M], e_{k} ; \sigma+B-A\right) \cdot n_{M}\left(f_{k}, a, e_{j} ; A\right) \otimes e^{-B}
\end{aligned}
$$

by Proposition 1.6(iv). But

$$
\begin{aligned}
Q_{\sigma} *_{M} a & =\sum_{C, k} n_{P}\left([M],[M], e_{k} ; \sigma+C\right) f_{k} *_{M} a \otimes e^{-C} \\
& =\sum_{C, A, k, j} n_{P}\left([M],[M], e_{k} ; \sigma+C\right) \cdot n_{M}\left(f_{k}, a, e_{j} ; A\right) f_{j} \otimes e^{-A-C} .
\end{aligned}
$$

The result follows.

Corollary $3.8 \Psi_{\phi, \sigma}\left(a *_{M} b\right)=\left(\Psi_{\phi, \sigma}(a)\right) *_{M} b$.

It follows from the above proposition that, for all sections $\sigma, Q_{\sigma}=\Psi_{\phi, \sigma}([M])$ is a unit in the ring $Q H_{e v}\left(M, \Lambda_{R}\right)$. We now define the map

$$
\rho: \pi_{1}(\operatorname{Ham}(M, \omega)) \rightarrow Q H_{*}\left(M, \Lambda_{R}\right)^{\times}
$$

by:

Proof of Theorem 1.5

$$
\rho(\phi)=Q_{\sigma_{\phi}}
$$

We have to show that $\rho$ is a homomorphism. Proposition 3.7 implies that

$$
\rho^{\prime}(\phi)(a)=\rho(\phi) *_{M} a,
$$

and by Proposition $3.4 \rho^{\prime}$ is a homomorphism. Hence result.

The next result will be useful in $§ 3.3$. 
Lemma $3.9 \rho(\phi)=\mu 11 \otimes e^{-A}$ if and only if

$$
\begin{aligned}
n_{P}\left(a, b,[M] ; \sigma_{\phi}+B\right) & =0, & & B \neq A \\
& =\mu a \cdot b, & & B=A .
\end{aligned}
$$

Proof: This is immediate from the definitions.

Following Seidel let us write

$$
Q H^{+}=\bigoplus_{k<n} H_{2 k} \otimes \Lambda_{R}, \quad \text { where } 2 n=\operatorname{dim} M .
$$

The next result follows immediately from the definitions.

Lemma 3.10 (i) If the Gromov-Witten invariants $n_{M}(a, b, c ; B)$ vanish whenever $c_{1}(B)>$ 0 then $Q H^{+}{ }_{*_{M}} Q H^{+} \subset Q H^{+}$.

(ii) If $Q H^{+} *_{M} Q H^{+} \subset Q H^{+}$then every unit in $Q H_{\text {ev }}^{\times}$has the form $11 \otimes \lambda+x$ for some unit $\lambda \in \Lambda_{R}$ and some $x \in Q H^{+}$.

Units in $Q H_{*}\left(P, \Lambda_{R}\right)$ of the form $\mathbb{1} \otimes \lambda+x$ are in some sense trivial. Observe that if a unit $\lambda=\sum_{B} \lambda_{B} e^{B} \in \Lambda_{R}$ has more than one nonzero coefficient $\lambda_{B}$ then either $\lambda$ itself or its inverse $\lambda^{-1}$ has infinitely many nonzero coefficients. It is not clear whether such units can occur as values of $\rho$. If so, it would mean that $P_{\phi}$ had nonvanishing Gromov-Witten invariants for infinitely many section classes.

Lemma 3.11 If $\rho(\phi)=11 \otimes \lambda+x$ where $\lambda \neq 0$ then $\bar{I}_{c}(\phi)=0$.

Proof: The hypothesis means that one of the numbers $n_{P}([M],[M], p t ; \sigma)$ is nonzero, which is possible only if $c_{\phi}(\sigma)=0$. Thus there is an (integral) section of $P \rightarrow S^{2}$ on which $c_{\phi}=0$, which immediately gives the result.

In [Sd1] 111 Seidel calculated $\Psi_{\phi, \sigma}$ in some cases, for example, when $\phi$ is rotation in the first coordinate of $\mathbf{C P}^{n}$, and when $\phi$ is rotation in the fiber of the rational ruled surface $\mathbf{F}_{2}$.

The next example is slightly easier because $H_{2}^{S}(M)$ has rank 1 . We will also calculate the homomorphisms $\bar{I}_{c}, \bar{I}_{u}$.

Example 3.12 Let $M$ be total space of the nontrivial $S^{2}$-bundle over $T^{2}$ and let $\phi$ be the loop that rotates once around in the fibers fixing the points of the sections $T_{ \pm}$of selfintersection \pm 1 . Then $P_{\phi}$ fibers over $Z=T^{2} \times S^{2}$ with fiber $F=S^{2}$, and $M \subset P_{\phi}$ can be identified with the inverse image of $T^{2} \times p t$. In fact $P$ can be can be thought of as the projectivization of $L \oplus \mathbf{C}$ where $L$ is a line bundle over $Z$ with $c_{1}(L)=\lambda+\mu$, where $\lambda, \mu$ are the obvious generators of $H^{2}(Z)$ with $\lambda\left(T^{2}\right)=1, \mu\left(S^{2}\right)=1$. We will use the same letters $\lambda, \mu$ for the pullbacks of these classes to $P$. There is another good class in $H^{2}(P)$ namely the vertical Chern class $\nu$ of the fibration $P \rightarrow Z$. This is 2 on the fiber $F$ and takes the values \pm 1 on $S_{ \pm}, T_{ \pm}$, where $T_{ \pm}, S_{ \pm}$are copies of $T^{2} \times p t$ and $p t \times S^{2}$ in the two obvious sections $Z_{ \pm}$of $P \rightarrow Z$.

The first claim is that the even part $H^{e v}(P)$ of $H^{*}(P)$ is generated by $\lambda, \mu, \nu$ with the relations $\lambda^{2}=\mu^{2}=0, \nu^{2}=2 \lambda \mu$. To see this, note that $\operatorname{PD}(\nu)=Z_{+}+Z_{-}$. Hence

$$
\operatorname{PD}\left(\nu^{2}\right)=\left(Z_{+}+Z_{-}\right)^{2}=\left(S_{+}+T_{+}\right)-\left(S_{-}+T_{-}\right)=2 F .
$$


Also $\operatorname{PD}(\mu)=M$ and $\operatorname{PD}(\lambda)=Z_{+}-Z_{-}-M$. To check:

$$
\begin{aligned}
\left(Z_{+}-Z_{-}-M\right)^{2} & =Z_{+}^{2}+Z_{-}^{2}-2 M\left(Z_{+}-Z_{-}\right) \\
& =\left(T_{+}+S_{+}\right)-\left(T_{-}+S_{-}\right)-2\left(T_{+}-T_{-}\right) \\
& =2 F-2 F=0 .
\end{aligned}
$$

Also $\operatorname{PD}(\lambda \mu)=M\left(Z_{+}-Z_{-}-M\right)=T_{+}-T_{-}=F$. (This is another check, since obviously $\operatorname{PD}(\lambda \mu)=F$. $)$

Let us calculate $\bar{I}_{c}(\phi)=c_{\phi}(\sigma)(\bmod N)$. In our case the minimal (spherical) Chern number of $M=2$. Further the classes $S_{ \pm}$are section classes of $P \rightarrow S^{2}$. Hence

$$
\bar{I}_{c}(\phi)=1 \in \mathbf{Z} / 2 \mathbf{Z} \text {. }
$$

Next, identify $H_{2}(M, \mathbf{R}) / H_{2}^{S}(M, \mathbf{R})$ with $\mathbf{R} T$ where $T=T_{+}=T_{-}$. Observe that

$$
\mathrm{PD}(\lambda \nu)=\left(Z_{+}-Z_{-}-M\right)\left(Z_{+}+Z_{-}\right)=\left(T_{+}+S_{+}+T_{-}+S_{-}\right)-T_{+}-T_{-}=S_{+}+S_{-}
$$

is spherical. So are $\operatorname{PD}\left(\nu^{2}\right)$ and $\operatorname{PD}(\lambda \mu)$, while $\operatorname{PD}(\mu \nu)=T_{+}+T_{-}$is not. Let us suppose that the symplectic form $\omega$ on $M$ is such that

$$
\omega\left(T_{-}\right)=\kappa, \quad \omega(F)=2 .
$$

Then $u_{\phi}=(1+\kappa) \lambda+\varepsilon \mu+\nu$ where $\varepsilon$ is chosen so that

$$
0=u_{\phi}^{3}=c^{3}(3(1+\kappa) \varepsilon+1)
$$

Hence

$$
\bar{I}_{u}(\phi)=2 \varepsilon \mathrm{PD}(\mu \nu)=4 \varepsilon T=\frac{-4}{3(1+\kappa)} T .
$$

Finally, let us calculate $\rho(\phi)$. The first step is to calculate $Q H_{e v}(M)$. Since the only spherical class in $H_{2}(M)$ is $F$, it is easy to check that the quantum products of the basis elements $[M]=11, F, T_{-},[p t]$ equal their usual intersection product except in the following cases:

$$
T_{-} * T_{-}=-[p t]+\mathbb{1} \otimes e^{-F}, \quad[p t] * T_{-}=F \otimes e^{-F} .
$$

Next, we need to find all Gromov-Witten invariants

$$
n_{P}([M],[M], a ; \sigma)=n_{P}([M], a ; \sigma)
$$

where $\sigma$ is a section class. Observe there is a complex structure $J$ on $P$ for which the submanifolds $Z_{ \pm}, T_{ \pm}, S_{ \pm}$are holomorphic, as are the projections $p_{Z}: P \rightarrow Z, p: P \rightarrow S^{2}$. Therefore, any $J$-holomorphic section $C$ of $P \rightarrow S^{2}$ projects to a holomorphic sphere $\bar{C}$ in $Z$ and hence lies in the ruled surface $p_{Z}^{-1}(\bar{C})$. It follows that $C$ must lie in a section class $\sigma=S_{-}+k F$ with $k \geq 0$. In particular, it is regular. Hence the moduli space $\overline{\mathcal{M}}_{0,2}(P, J, \sigma)$ carries a fundamental class of the right dimension, and one can calculate $n_{P}([M], a ; \sigma)$ by naively counting curves. In particular, the set of $J$-holomorphic curves in class $S_{-}$fills out $Z_{-}$and so

$$
n_{P}\left([M], F ; S_{-}\right)=1, \quad n_{P}\left([M], T_{-} ; S_{-}\right)=-1, \quad n_{P}\left([M], a ; S_{+}\right)=0,
$$


where the last equality holds for dimensional reasons: $n_{P}([M], a ; \sigma) \neq 0$ only if $c_{\phi}(\sigma) \leq 0$ by equation (3) in $\S 2.1$. Hence

$$
\Psi_{\phi, S_{-}}[M]=T_{-} .
$$

(This also follows by applying [Sd1] Proposition 7.11.)

Now observe that $M$ is spherically monotone, and $\mathcal{H}=\mathbf{Z}$ is generated by $F$. The class $\sigma_{\phi}$ must have the form $S_{-}+\delta F$ where, by Lemma 3.2(ii) $u_{\phi}\left(\sigma_{\phi}\right)=0$. Hence

$$
\sigma_{\phi}=S_{-}+\delta F, \quad \text { where } \delta=\frac{4+3 \kappa}{6+6 \kappa} .
$$

Thus

$$
\rho(\phi)=T_{-} \otimes e^{\delta F}
$$

This is a unit in $Q H_{e v}\left(M, \Lambda_{R}\right)$ of infinite order. Hence $\phi$ itself must have infinite order. Observe finally that $c_{\phi}\left(\sigma_{\phi}\right) \neq 0$. This is no contradiction since we are in a degenerate case of Lemma 3.2.

\subsection{The vertical quantum cohomology of $P$}

Additively $Q H_{V}^{*}\left(P, \Lambda_{R}^{*}\right)=H^{*}\left(P ; \Lambda_{R}^{*}\right)$, where $\Lambda_{R}^{*}$ is the Novikov ring of $\mathcal{H}_{R}=\mathcal{H}_{R}(M)$ that satisfies the finiteness condition dual to that defining $\Lambda_{R}$. Thus the elements of $\Lambda_{R}^{*}$ have the form $\sum_{B \in \mathcal{H}_{R}} \lambda_{B}^{*} e^{B}$ where, for each $\kappa>0$, there are only finitely many nonzero coefficients $\lambda_{B}^{*}$ with $\omega(B)<\kappa$. The degree of $e^{B}$ is defined to be $2 c_{1}(B)$ as before.

Recall that the quantum cup product on $M$ is the Poincaré dual to the quantum intersection product on $M$. In detail: there is a dual pairing

$$
Q H^{*}\left(M, \Lambda_{R}^{*}\right) \otimes Q H_{*}\left(M, \Lambda_{R}\right) \rightarrow \mathbf{Q}:\left\langle\sum \alpha_{A}^{*} \otimes e^{A}, \sum a_{B} \otimes e^{-B}\right\rangle=\sum_{B}\left\langle\alpha_{B}^{*}, a_{B}\right\rangle,
$$

and Poincaré duality $\mathrm{PD}=\mathrm{PD}_{M}$ is given by:

$$
\mathrm{PD}: Q H^{*}\left(M, \Lambda_{R}^{*}\right) \rightarrow Q H_{*}\left(M, \Lambda_{R}\right): \quad \sum \alpha_{A} \otimes e^{A} \mapsto \sum \mathrm{PD}\left(\alpha_{A}\right) \otimes e^{-A} .
$$

The quantum cup product on $M$ is defined by

$$
\alpha * \beta=\sum_{B}(\alpha * \beta)_{B} \otimes e^{B},
$$

where

$$
\left\langle(\alpha * \beta)_{B}, c\right\rangle=n_{M}(\operatorname{PD}(\alpha), \operatorname{PD}(\beta), c ; B),
$$

and it is not hard to check that

$$
\mathrm{PD}(\alpha * \beta)=\mathrm{PD}(\alpha) * \mathrm{PD}(\beta) .
$$

The vertical quantum product $*_{V}$ on $Q H^{*}\left(P, \Lambda_{R}^{*}\right)$ is defined similarly.

Theorem 1.1 implies that the Wang sequence in rational cohomology decomposes as a family of exact sequences:

$$
0 \rightarrow H^{*-2}(M) \stackrel{\iota}{\rightarrow} H^{*}\left(P_{\phi}\right) \stackrel{\iota}{\rightarrow} H^{*}(M) \rightarrow 0,
$$


where $\iota$ ! $H^{*-2}(M) \rightarrow H^{*}(P)$ is the cohomology transfer:

$$
\iota !(\alpha)=\mathrm{PD}_{P}\left(\iota_{*}\left(\mathrm{PD}_{M}(\alpha)\right)\right) .
$$

Observe that $\iota^{*}$ is a ring homomorphism while $\iota$ ! satisfies the identity:

$$
\iota !(\alpha \cup \beta)=s(\alpha) \cup \iota !(\beta),
$$

where $s: H^{*}(M) \rightarrow H^{*}(P)$ is any right inverse to $\iota^{*}$, i.e. any map such that $\iota^{*} \circ s=$ id. Here is the quantum analog.

Proposition 3.13 There is an exact sequence

$$
0 \rightarrow Q H^{*-2}\left(M, \Lambda_{R}^{*}\right) \stackrel{\iota}{\rightarrow} Q H_{V}^{*}\left(P_{\phi}, \Lambda_{R}^{*}\right) \stackrel{\iota^{*}}{\rightarrow} Q H^{*}\left(M, \Lambda_{R}^{*}\right) \rightarrow 0,
$$

of $\Lambda_{R}^{*}$-modules where $\iota^{*}$ is a ring homomorphism with respect to the quantum products and !! satisfies the identity

$$
\iota_{!}\left(\alpha *_{M} \beta\right)=s(\alpha) *_{V} \iota !(\beta),
$$

for any additive splitting $s$ as above.

Proof: This follows by dualizing Proposition 1.9.

Remark 3.14 This proposition continues to hold whenever $P \rightarrow S^{d}$ is a fibration with structural group $\operatorname{Ham}(M)$. In fact, it is shown in [LMP3] that the cohomology of $P$ is still additively split in this case. It is not hard to check that all that is then needed to establish the existence of the vertical quantum multiplication is the fiberwise symplectic structure of $P$. Seidel notes in [Sd2] that the Wang sequence (8) with base $S^{d}$ can be interpreted as saying that the ring $H^{*}\left(P_{\phi}\right)$ is a (first order) deformation of $H^{*}(M)$. (Here $\phi \in \pi_{d-1}(\operatorname{Ham}(M))$.) He considers only the case when $M=\mathbf{C P}^{m} \times \mathbf{C P}^{n}$ and then looks at the part of the vertical quantum product $*_{V}$ that is given by counting vertical $A$-curves, where $A$ is the positive generator of $H_{2}\left(\mathbf{C P}^{n}, \mathbf{Z}\right)$. His equation (5.1) is the analog of the statement that

$$
\iota^{*}: Q H_{V}^{*}\left(P_{\phi}, \Lambda_{R}^{*}\right) \longrightarrow Q H^{*}\left(M, \Lambda_{R}^{*}\right)
$$

a ring homomorphism with respect to the quantum products. The crucial fact in his case is that the moduli space of spheres in class $A$ is compact, which means that his operation gives rise to an associative multiplication on $\widetilde{R} \otimes F[q] / q^{2}$ : cf. property $\left(1^{\prime}\right)$ in [Sd2] Definition 4.1. More generally, it would be sufficient to require that in the decomposition

$$
n_{P}(u, v, w, z ; \iota(A))=\sum_{A=A_{1}+A_{2}} n_{P}\left(u, v, e_{\alpha}^{\prime} ; \iota\left(A_{1}\right)\right) \cdot n_{P}\left(f_{\alpha}^{\prime}, w, z ; \iota\left(A_{2}\right)\right),
$$

the only nonzero terms have $A_{1}$ or $A_{2}$ equal to 0 . 


\subsection{The ring structure of $H^{*}\left(P_{\phi}\right)$}

In this section we discuss conditions under which the ring $H^{*}(P)$ splits as the product of $H^{*}(M)$ with $H^{*}\left(S^{2}\right)$. This happens if and only if there is a ring homomorphism

$$
r: H^{*}(M) \rightarrow H^{*}(P),
$$

such that $\iota^{*} \circ r=$ id. Note that in this situation $u_{\phi}=r([\omega])$ since $r([\omega])^{n+1}=r\left([\omega]^{n+1}\right)=0$. Conversely, we have the following elementary result.

Lemma 3.15 If $H^{*}(M, \mathbf{R})$ is generated as a ring by $[\omega]$, then the ring $H^{*}(P)=H^{*}(P, \mathbf{Q})$ splits.

Proof: Since the hypothesis implies that $H^{2}(M)$ has dimension 1, we can assume without loss of generality that $\omega$ is rational. It now suffices to define the splitting $r$ by $r\left([\omega]^{k}\right)=u_{\phi}^{k}$.

It is tempting to try to generalize this to the case when $H^{*}(M)$ is generated by $H^{2}(M)$. However, in this case the ring $H^{*}(P)$ does not always split. For example, one could calculate $H^{*}(P)$ as in Example 3.12 but taking $M$ to be equal to the nontrivial ruled surface over $S^{2}$ (rather than $T^{2}$ ) so that $H^{2}(M)$ does generate $H^{*}(M)$. See also the examples in [Sd2] which have fiber $\mathbf{C P}^{m} \times \mathbf{C P}^{n}$.

Our next aim is to prove that Theorem 1.2 holds. Thus we have to show that the ring $H^{*}(P)$ splits under the assumption that all vertical Gromov-Witten invariants

$$
n_{P}\left(v_{1}, \ldots, v_{k} ; B\right), B \neq 0, k \leq 4,
$$

vanish. Since this implies that the quantum multiplication on $M$ is trivial, we must have

$$
\rho(\phi)=\mathbb{1} \otimes \lambda+x
$$

as in Lemma 3.10. Choose $A$ so that the term $\lambda_{A}=\mu$ in the expansion

$$
\lambda=\sum_{B} \lambda_{B} e^{-B}
$$

is nonzero. Then, if $\sigma_{A}=\sigma_{\phi}+A$,

$$
n_{P}\left(a, b,[M] ; \sigma_{A}\right)=\mu a \cdot b .
$$

The following remark will be useful later.

Lemma 3.16 If all the vertical Gromov-Witten invariants with $B \neq 0$ vanish for $P=P_{\phi}$, then the same is true for $P_{\phi^{-1}}$.

Proof: As noted in the proof of Corollary 2.6, there is a fiberwise diffeomorphism $h$ from $P_{\phi}$ to $P_{\phi^{-1}}$ that is a symplectomorphism on the fibers and covers an orientation reversing map on the bases. Hence, given any fibered tame $J$ on $P_{\phi}$, there is a fibered tame almost complex structure $J^{\prime}$ on $P_{\phi^{-1}}$ that restricts on the fibers to the pushforward $h_{*}(J)$. Therefore, $h$ maps 
vertical $J$-holomorphic curves on $P$ to vertical $J^{\prime}$-holomorphic curves in $P^{\prime}$.9 Moreover, as in $\S 4.3$, the regularization $\overline{\mathcal{M}}^{\nu}=\overline{\mathcal{M}}_{0, k}^{\nu}(P, J, B)$ used to calculate the vertical Gromov-Witten invariants of $P$ can be constructed using this $J$ and a fibered perturbation term $\nu$. Hence the pushforward by $h$ of $\overline{\mathcal{M}}^{\nu}$ is a regularization of $\overline{\mathcal{M}}_{0, k}\left(P^{\prime}, J^{\prime}, h(B)\right)$ and can be used to calculate the vertical Gromov-Witten invariants of $P^{\prime}$. Thus

$$
n_{P}\left(v_{1}, \ldots, v_{k} ; B\right)=n_{P^{\prime}}\left(h_{*}\left(v_{1}\right), \ldots, h_{*}\left(v_{k}\right) ; h_{*}(B)\right) .
$$

The result is now immediate.

Lemma 3.17 The ring $H^{*}(P)$ splits if and only if there is an additive homomorphism $s: H_{*}(M) \rightarrow H_{*+2}(P)$ such that, for all $a, b, c \in H_{*}(M)$,

$$
\begin{array}{ll}
\text { (i) } & s(a) \cap[M]=a, \\
\text { (ii) } & (s(a) \cap s(b)) \cdot s(c)=0 .
\end{array}
$$

Proof: (i) implies that for each $a, b$ there is $x \in H_{*}(M)$ such that

$$
s(a) \cap s(b)=s(a \cap b)+\iota(x) .
$$

Further $s([M])=[P]$. Hence, taking $b=[M]$ in (ii), we find that $s(a) \cdot s(c)=0$ for all $a, c$. Applying (ii) again, we have $s(a) \cap s(b)=s(a \cap b)$ for all $a, b$. One now defines an inclusion $r$ of the ring $H^{*}(M)$ into $H^{*}(P)$ such that $\iota^{*} \circ r=\operatorname{id}_{M}$ by setting

$$
r(\alpha)=\mathrm{PD}_{P}\left(s\left(\mathrm{PD}_{M}(\alpha)\right) .\right.
$$

The converse is similar.

Under the above hypotheses on $P$, define $s=s_{A}: H_{*}(M) \rightarrow H_{*+2}(P)$ by the identity

$$
s_{A}(a) \cdot{ }_{P} v=\frac{1}{\mu} n_{P}\left(a,[M], v ; \sigma_{A}\right), \quad v \in H_{*}(P) .
$$

Since

$$
\mu\left(s_{A}(a) \cap[M]\right) \cdot{ }_{M} b=\mu s_{A}(a) \cdot{ }_{P} b=n_{P}\left(a, b,[M] ; \sigma_{A}\right),
$$

it is clear that

$$
s_{A}(a) \cap[M]=a .
$$

Therefore, we just have to show that

$$
s_{A}(a) \cdot s_{A}(b)=0, \quad\left(s_{A}(a) \cap s_{A}(b)\right) \cdot s_{A}(c)=0 .
$$

To get a handle on these intersections, we now show that under the given hypotheses they can be interpreted in terms of Gromov-Witten invariants.

Lemma 3.18 With the hypotheses of Theorem 1.2,

(i) $\mu s_{A}(a) \cdot{ }_{P} s_{A}(b)=n_{P, \chi}\left(s_{A}(a), s_{A}(b),[M],[M] ; \sigma_{A}\right)$;

(ii) $n_{P, \chi}\left(s_{A}(a), s_{A}(b),[M],[M] ; \sigma_{A}\right)=0$.

\footnotetext{
${ }^{9}$ As Seidel pointed out to me, there is no similar correspondence between curves that represent section classes, since $h$ is antiholomorphic in the horizontal directions.
} 
Proof: Let $s$ be any splitting that satisfies the conditions of Lemma 3.5. Using the composition rule (6) we find that

$$
\begin{gathered}
n_{P, \chi}\left(s_{A}(a), s_{A}(b),[M],[M] ; \sigma_{A}\right) \\
=\sum_{B, i} n_{P}\left([M],[M], e_{i} ; \sigma_{A}-B\right) \cdot n_{P}\left(s\left(f_{i}\right), s_{A}(a), s_{A}(b) ; B\right)+ \\
\quad n_{P}\left([M],[M], s\left(e_{i}\right) ; \sigma_{A}-B\right) \cdot n_{P}\left(f_{i}, s_{A}(a), s_{A}(b) ; B\right),
\end{gathered}
$$

since all other terms such as

$$
n_{P}\left([M],[M], e_{i} ; B\right) \cdot n_{P}\left(s\left(f_{i}\right), s_{A}(a), s_{A}(b) ; \sigma_{A}-B\right)
$$

that occur in the expansion vanish by Proposition 1.6. By hypothesis, the only nonzero terms have $B=0$. For terms of the first kind, a dimension count shows that $e_{i}=[p t]$. Thus

$$
\begin{aligned}
\sum_{i} n_{P} & \left([M],[M], e_{i} ; \sigma_{A}\right) \cdot n_{P}\left(s\left(f_{i}\right), s_{A}(a), s_{A}(b) ; 0\right) \\
& =n_{P}\left([M],[M],[p t] ; \sigma_{A}\right) \cdot n_{P}\left([P], s_{A}(a), s_{A}(b) ; 0\right) \\
& =\mu s_{A}(a) \cdot s_{A}(b)
\end{aligned}
$$

Further, when $B=0$ in terms of the second kind, they have to vanish for reasons of dimension. This proves (i).

Now let $a=e_{j}, b=e_{k}$. We may suppose that $\operatorname{dim}\left(e_{j}\right) \equiv \operatorname{dim}\left(e_{k}\right) \equiv \operatorname{dim}\left(e_{i}\right) \equiv \varepsilon \bmod 2$ since otherwise all terms below vanish. Grouping the terms differently, we have

$$
\begin{aligned}
& n_{P, \chi}\left(s_{A}\left(e_{j}\right), s_{A}\left(e_{k}\right),[M],[M] ; \sigma_{A}\right) \\
& \quad=\sum_{B, i} n_{P}\left(s_{A}\left(e_{j}\right),[M], e_{i} ; \sigma_{A}-B\right) \cdot n_{P}\left(s\left(f_{i}\right), s_{A}\left(e_{k}\right),[M] ; B\right)+ \\
& \quad(-1)^{\varepsilon} n_{P}\left(s_{A}\left(e_{j}\right),[M], s\left(f_{i}\right) ; B\right) \cdot n_{P}\left(e_{i}, s_{A}\left(e_{k}\right),[M] ; \sigma_{A}-B\right),
\end{aligned}
$$

where the sign $(-1)^{\varepsilon}$ is needed because we have reversed the order of $e_{i}, s\left(f_{i}\right)$ in the basis. When $B=0$,

$$
n_{P}\left(s\left(f_{i}\right), s_{A}\left(e_{k}\right),[M] ; B\right)=n_{M}\left(f_{i}, e_{k},[M] ; B\right)=(-1)^{\varepsilon} \delta_{i k} .
$$

Hence

$$
\begin{aligned}
n_{P, \chi}( & \left.s_{A}\left(e_{j}\right), s_{A}\left(e_{k}\right),[M],[M] ; \sigma_{A}\right) \\
& =(-1)^{\varepsilon} n_{P}\left(s_{A}\left(e_{j}\right),[M], e_{k} ; \sigma_{A}\right)+(-1)^{\varepsilon} n_{P}\left(e_{j}, s_{A}\left(e_{k}\right),[M] ; \sigma_{A}\right) \\
& =\mu s_{A}\left(e_{k}\right) \cdot s_{A}\left(e_{j}\right)+(-1)^{\varepsilon} \mu s_{A}\left(e_{j}\right) \cdot s_{A}\left(e_{k}\right) \\
& =(-1)^{\varepsilon} 2 \mu s_{A}\left(e_{j}\right) \cdot s_{A}\left(e_{k}\right),
\end{aligned}
$$

where the second equality uses the definition of $s_{A}$. This is consistent with (i) only if $s_{A}\left(e_{j}\right) \cdot s_{A}\left(e_{k}\right)=0$ for all $j, k$.

Hence the splitting $s_{A}$ satisfies the conditions of Lemma 3.5, and, for simplicity, we now write it as $s(a)$ instead of $s_{A}(a)$. 
Lemma 3.19 With the hypotheses of Theorem 1.2,

$$
n_{P}\left(s(a), b, c ; \sigma_{A}\right)=0
$$

for all $a, b, c \in H_{*}(M)$.

Proof: By Proposition 1.6(iv),

$$
\begin{aligned}
n_{P}\left(s(a), b, c ; \sigma_{A}\right)= & \sum_{B, i} n_{P}\left(s(a),[M], e_{i} ; \sigma_{A}-\iota(B)\right) \cdot n_{M}\left(f_{i}, b, c ; B\right)+ \\
& n_{P}\left([M],[M], e_{i} ; \sigma_{A}-\iota(B) \cdot n_{M}\left(f_{i}, a, b, c ; B\right) .\right.
\end{aligned}
$$

But when $B=0$, terms of the first kind vanish by the previous lemma, since, by definition,

$$
n_{P}\left(s(a),[M], e_{i} ; \sigma_{A}\right)= \pm s\left(e_{i}\right) \cdot s(a) .
$$

Terms of the second kind also vanish for dimensional reasons, since $n_{P}\left(s(a), b, c ; \sigma_{A}\right) \neq 0$ only if

$$
\operatorname{dim}(a)+\operatorname{dim}(b)+\operatorname{dim}(c)+2=4 n,
$$

which implies that the triple intersection $(a \cap b) \cdot c=0$. Note that here we need to know that a 4-point vertical invariant is zero when $B \neq 0$.

Lemma 3.20 With the hypotheses of Theorem 1.9 and notation as above,

(i) $s(a) \cdot_{P}(s(b) \cap s(c))= \pm \mu n_{P, \chi}\left(a, s(b), s(c),[M] ; \sigma_{A}\right)$;

(ii) $n_{P, \chi}\left(a, s(b), s(c),[M] ; \sigma_{A}\right)=0$.

Proof: As in Lemma 3.18

$$
\begin{aligned}
n_{P, \chi}\left(a, s(b), s(c),[M] ; \sigma_{A}\right)=\sum_{i} n_{P}\left(a,[M], e_{i} ; \sigma_{A}\right) \cdot n_{P}\left(s\left(f_{i}\right), s(b), s(c) ; 0\right) \\
\\
+n_{P}\left(a,[M], s\left(e_{i}\right) ; \sigma_{A}\right) \cdot n_{P}\left(f_{i}, s(b), s(c) ; 0\right) .
\end{aligned}
$$

Terms of the second kind vanish by Lemma 3.18. Also, because $n_{P}\left(a, b,[M] ; \sigma_{A}\right)=\mu a \cdot b$, terms of the first kind sum to give $(-1)^{\varepsilon} \mu s(a) \cdot P(s(b) \cap s(c))$ where $\varepsilon=\operatorname{dim}(a)$. This proves (i).

We may also write

$$
\begin{aligned}
& n_{P, \chi}\left(a, s(b), s(c),[M] ; \sigma_{A}\right) \\
& =\sum_{B, i} n_{P}\left(a, s(b), e_{i} ; \sigma_{A}-B\right) \cdot n_{P}\left(s\left(f_{i}\right), s(c),[M] ; B\right)+ \\
& \quad n_{P}\left(a, s(b), s\left(e_{i}\right) ; B\right) \cdot n_{P}\left(f_{i}, s(c),[M] ; \sigma_{A}-B\right) .
\end{aligned}
$$

Again, $n_{P}\left(s\left(f_{i}\right), s(c),[M] ; B\right)=n_{M}\left(f_{i}, c,[M] ; B\right) \neq 0$ only if $B=0$. Since we always have $n_{P}\left(a, s(b), e_{i} ; \sigma_{A}\right)=0$ by Lemma 3.19, terms of the first kind vanish. A similar argument shows that terms of the second kind vanish.

Here is a more precise version of Theorem 1.2 . 
Proposition 3.21 Suppose that all vertical k-point Gromov-Witten invariants of $P=P_{\phi}$ with $B \neq 0$ and $k \leq 4$ vanish. Then:

(i) the ring $H^{*}(P)$ splits;

(ii) $\bar{I}_{c}(\phi)=\bar{I}_{u}(\phi)=0$;

(iii) $\rho(\phi)=\mu \mathbb{1}+x$ for some $\mu \in \mathbf{Q}, x \in Q H^{+}(M)$.

Proof: The first statement follows from Lemmas 3.18 and 3.20. Now consider the ring homomorphism $r: H^{*}(M) \rightarrow H^{*}(P)$ Poincaré dual to $s$. Thus

$$
r(\alpha)=\mathrm{PD}_{P}\left(s\left(\mathrm{PD}_{M}(\alpha)\right), \quad \text { and } \iota^{*} \circ r(\alpha)=\alpha .\right.
$$

Observe that the coupling class $u_{\phi}$ must equal $r([\omega])$ since $r\left([\omega]^{n+1}\right)=0$ for dimensional reasons. Hence

$$
\operatorname{PD}_{P}\left(u_{\phi}^{n}\right)=s\left(\mathrm{PD}_{M}\left(\omega^{n}\right)\right)=s([p t]) .
$$

Now $s([p t])$ is represented by a union of sections in the (equivalence) class $\sigma_{A}$. Hence it is spherical. Thus $\operatorname{PD}_{P}\left(u_{\phi}^{n}\right)$ is spherical, so that $\bar{I}_{u}(\phi)=0$. Moreover $u_{\phi}\left(\sigma_{A}\right)=u_{\phi}^{n+1}([P])=0$. Since we already know that $c_{\phi}\left(\sigma_{A}\right)=0$ for dimensional reasons, we must have $\sigma_{A}=\sigma_{\phi}$. (Note that this conclusion holds even in the degenerate cases considered in Lemma 3.2 (ii).) Thus (ii) holds and $\rho(\phi)$ has the form $\mu 11+x$.

Remark 3.22 If there is a fibered $J$ with no vertical $J$-holomorphic curves at all, one can show in addition that $\mu= \pm 1$. The point is that the moduli space $\mathcal{M}=\mathcal{M}\left(P, J, \sigma_{A}\right)$ of unparametrized spheres in class $\sigma_{A}$ is now a compact manifold and $\mu$ is the degree of the evaluation map

$$
\text { ev }: \mathcal{M} \times S^{2} \rightarrow P .
$$

(ev is well defined since the elements in $\mathcal{M}$ can be parametrized as sections.) Hence $\mu$ is an integer. The proof of Lemma 3.16 shows that if $P=P_{\phi}$ has no vertical curves, the same is true for $P^{\prime}=P_{\phi^{-1}}$. Since $\rho\left(\phi^{-1}\right)=(1 / \mu) \mathbb{1}+x^{\prime}$, we must have $\mu= \pm 1$.

The above arguments prove that the ring $H^{*}(P)$ splits if there are no nontrivial vertical invariants. We shall now show that they lead to the same conclusion either under a strong hypothesis on the horizontal invariants or under a "mixed" hypothesis, that assumes less about the horizontal invariants but more about the vertical ones. Recall from $\S 1.3$ that, given any section class, the horizontal quantum product $*_{H, \sigma}$ is defined by

$$
\left(u *_{H, \sigma} v\right) \cdot w=\sum n_{P}(u, v, w ; \sigma+B) \otimes e^{-B} .
$$

In particular, $\rho(\phi)=\left([M] *_{H, \sigma_{\phi}}[M]\right) \cap[M]$. Hence hypothesis (i) below implies that $\rho(\phi)=$ $\mu 11 \otimes e^{-A}$.

Proposition 3.23 The conclusions of Proposition 3.21 hold under each of the following assumptions.

(i) There is a section class $\sigma_{A}$ such that $[M] *_{H, \sigma_{A}}[M]=\mu[P]$; or

(ii) The 3-point invariants $n_{M}(a, b, c ; B), B \neq 0$ vanish and $\rho(\phi)=\mu 11 \otimes e^{-A}$.

In particular, in each case the ring $H^{*}(P)$ splits as a product of $H^{*}(M)$ and $H^{*}\left(S^{2}\right)$. 
Proof: In both cases, one can run through the proofs of Lemmas 3.18, 3.19 and 3.20, checking that the only nonzero terms in the expansions have to have $B=0$. Then the previous arguments apply.

For example, consider the first expansion in the proof of Lemma 3.18(i) and suppose that condition (ii) above holds. Then, because $\rho(\phi)=\mu 1 \otimes e^{-A}$,

$$
n_{P}\left(a,[M], e_{i} ; \sigma_{A}-B\right) \neq 0 \Longrightarrow B=0 .
$$

Thus $B=0$ for terms of the first kind. For terms of the second kind one uses the identity

$$
n_{P}\left(f_{i}, s_{A}(a), s_{A}(b) ; B\right)=n_{M}\left(f_{i}, a, b ; B\right)
$$

from Proposition 1.6(ii).

Similar arguments work under condition (i) since this is equivalent to assuming that

$$
n_{P}\left([M],[M], v ; \sigma_{A}-B\right) \neq 0 \Longrightarrow B=0, v=k[p t] .
$$

To end this section, we now show that the trivial bundle satisfies a strong form of condition (i) above that is sufficient to imply

$$
Q H^{*}\left(M \times S^{2}\right)=Q H^{*}(M) \otimes Q H^{*}\left(S^{2}\right),
$$

as claimed in Proposition 1.11. Here $Q H^{*}$ denotes the small quantum cohomology ring described in $\S 3.2$, with coefficients taken either in the usual Novikov ring $\Lambda_{X}$ or in the real ring $\Lambda_{R, X}$. Observe that $\Lambda_{M \times S^{2}} \cong \Lambda_{M} \otimes \Lambda_{S^{2}}$.

Let $s: H_{*}(M) \rightarrow H_{*+2}(P)$ be the obvious splitting,

$$
s(a)=a \times\left[S^{2}\right] .
$$

A proof of the following lemma is sketched in $\S 4.3 .2$ : see Lemma 4.16.

Lemma 3.24 Let $P=M \times S^{2}$ and $\sigma_{0}$ be the section class $\left[p t \times S^{2}\right]$. Then, for all $a \in H_{*}(M)$ and $u, v, w \in H_{*}(P)$,

(i) $n_{P}(u, v, w ; D)=0$ unless $D=k \sigma_{0}+B$ for some $B \in H_{2}(M)$ and $k=0,1$.

(ii) $n_{P}\left(s(a), u, v ; \sigma_{0}+B\right)=0$.

Proposition 3.25 If the conclusions of Lemma 3.24 hold, then

$$
Q H^{*}\left(M \times S^{2}\right)=Q H^{*}(M) \otimes Q H^{*}\left(S^{2}\right) .
$$

Proof: By Proposition 2.2, $\rho=11$ for the constant loop. Hence by definition (see Lemma 3.9), $n_{P}\left(a, b,[M], \sigma_{O}+B\right)=0$ if $B \neq 0$ or if $a \cdot b \neq 0$. It follows easily from Proposition 1.6 (iv) that

$$
n_{P}\left(a, b, c ; \sigma_{0}+B\right)=n_{M}(a, b, c ; B) .
$$

Part (ii) of the preceding lemma now implies that the part of the quantum product in $P$ coming from the classes $k \sigma_{0}+B, k=0,1$, agrees with that in $Q H^{*}(M) \otimes Q H^{*}\left(S^{2}\right)$. But, by (i), classes with $k \neq 0,1$ do not contribute to $Q H^{*}(P)$. Since they do not contribute to $Q H^{*}(M) \otimes Q H^{*}\left(S^{2}\right)$ either, the result follows. 


\subsection{The nonsqueezing theorem}

First of all, let us calculate the area $\alpha$ of $(P, \Omega)$. Note that if $u_{S}$ is the positive generator of $H^{2}\left(S^{2}, \mathbf{Z}\right)$ then $[\Omega]=u_{\phi}+\kappa p^{*}\left(u_{S}\right)$, for some $\kappa>0$.

Lemma 3.26 If $[\Omega]=u_{\phi}+\kappa p^{*}\left(u_{S}\right)$, then the area of $\alpha$ of $(P, \Omega)$ equals $\kappa$.

Proof: We can write $\Omega=\tilde{\omega}+\kappa p^{*}\left(\omega_{S}\right)$ where $[\tilde{\omega}]=u_{\phi}$ and $\left[\omega_{S}\right]=u_{S}$. Then

$$
\int_{P} \Omega^{n+1}=\int_{P}(n+1) \kappa \tilde{\omega}^{n} \wedge p^{*}\left(\omega_{S}\right)=(n+1) \kappa \int_{M} \omega^{n} \int_{S^{2}} \omega_{S},
$$

where the second equality holds because, at each point $x \in P, \Omega$ restricts to $p^{*}\left(\omega_{S}\right)$ on the 2-dimensional subspace $H_{x}$ of $T_{x} P$ that is $\Omega$-orthogonal to the tangent space to the fibers. The result follows.

Proposition 3.27 The nonsqueezing theorem holds if

$$
n_{P}\left([M],[M],[p t] ; \sigma_{\phi}\right) \neq 0 .
$$

Proof: The hypothesis implies that the evaluation map

$$
e v_{1}: \overline{\mathcal{M}}_{0,2}\left(P, J, \sigma_{\phi}\right) \rightarrow P
$$

has nonzero degree, and hence is surjective (where $e v_{1}$ evaluates at the first marked point $z_{1}$ ). Therefore, the usual proof of the nonsqueezing theorem (see Gromov $[\mathrm{G}]$ or $[\mathrm{LM}]$ ) implies that the radius $r$ of any symplectically embedded ball in $P$ is constrained by the inequality

$$
\pi r^{2} \leq \int_{\sigma_{\phi}} \Omega
$$

By the previous lemma, $[\Omega]=u_{\phi}+\alpha p^{*}\left(u_{S}\right)$. Moreover, by Lemma 3.2, we chose $u_{\phi}$ so that $u_{\phi}\left(\sigma_{\phi}\right)=0$ except in the degenerate case when $\omega=0$ on $H_{2}^{S}(M)$ on $H_{2}^{S}(M)$. This shows that $\pi r^{2} \leq \alpha$ in all cases except possibly this degenerate one.

To deal with this case, note that if $\omega=0$ on $H_{2}^{S}(M)$ then the hypothesis in Proposition 3.23 holds and $\sigma_{\phi}$ is the Poincaré dual of $u_{\phi}^{n}$. Hence we must still have $u_{\phi}\left(\sigma_{\phi}\right)=0$.

Corollary 3.28 The nonsqueezing theorem holds under the hypotheses of Theorem 1.2 and under those of Proposition 3.2.3.

Proof: By Proposition $3.21 n_{P}\left([M],[M],[p t] ; \sigma_{\phi}\right) \neq 0$ in these cases. 


\section{Gromov-Witten invariants in fibered spaces}

It remains to prove the properties of the Gromov-Witten invariants claimed in Proposition 1.6 and in Claim 2.11 and Lemma 2.12 of $\S 2.3$. No doubt, any of the approaches to defining general Gromov-Witten invariants can be adapted to do this. We will follow the Liu-Tian [LiuT1,2] method since in some way this is the most geometric. The idea is to perturb the moduli space $\overline{\mathcal{M}}_{0, k}(P, J, A)$ to a compact space called $\overline{\mathcal{M}}^{\nu}=\overline{\mathcal{M}}_{0, k}^{\nu}(P, J, A)$ that is sufficiently like a closed manifold of dimension $d$ to carry a rational fundamental class. This gives us a virtual moduli cycle whose elements are stable maps satisfying a perturbed Cauchy-Riemann equation. We can then argue geometrically using this cycle. We have given a detailed description of the construction of the virtual moduli cycle $\overline{\mathcal{M}}^{\nu}$ in [Mc3]. In $§ 4.1,2$ we will try to summarize enough of this discussion to make the the rest of the argument in $\S 4.3$ intelligible.

One of the key properties we need to establish concerns the relation between the invariants in class $\iota(A)$ in $P$ and those of class $A$ in $M$. For this, we first need to show that $\overline{\mathcal{M}}^{\nu}$ can be constructed using a fibered $J$ and a perturbation term $\nu$ that is compatible with the fibration $P \rightarrow S^{2}$. Then we must look carefully at how the stable maps in $M$ sit inside the full cycle $\overline{\mathcal{M}}^{\nu}$. In general, they form a codimension 2 subobject. We will give a rather $a d$ hoc definition of the structure put on $\overline{\mathcal{M}}^{\nu}$ that takes this into account: see Definition 4.3 .

\subsection{Branched pseudomanifolds}

In this section we describe the topological nature of the virtual cycle. We will work in the category of partially smooth topological spaces $Y$. Thus $Y$ is a Hausdorff topological space that is the image of a continous bijection

$$
i_{Y}: Y_{s m} \rightarrow Y,
$$

where $Y_{s m}$ is a finite union of open disjoint (Banach) manifolds. (Another way to think of $Y$ is as a space with two topologies.) A morphism $f: Y \rightarrow X$ between two objects in this category is a continuous map $f: Y \rightarrow X$ such that the induced map $f: Y_{s m} \rightarrow X_{s m}$ is smooth. Usually, we will be interested in the case when $X$ is a manifold, i.e. when $i_{X}$ is a homeomorphism, so that $X$ can be identified with the manifold $X_{s m}$. Morphisms $f: Y \rightarrow X$ will be called partially smooth maps, or, for short, simply maps. The path components of $Y_{s m}$ are sometimes called strata and sometimes components. If $Y$ is infinite-dimensional, strata that are open subsets of $Y$ are called top strata.

Definition 4.1 A (closed, oriented) branched pseudomanifold $Y$ of dimension $d$ is a compact partially smooth space $Y_{s m} \rightarrow Y$ where the components of $Y_{s m}$ have dimension at most $d$. The components of dimension $d$ are called $M_{i}$, those of dimension $(d-1)$ are called $B_{j}$, and $Y_{\leq k}$ denotes the union of all components of $Y_{s m}$ of dimension $\leq k$. Write:

$$
Y^{\text {top }}=\bigcup_{i} M_{i}, \quad B=\bigcup_{j} B_{j}, \quad Y^{\text {sing }}=Y-\left(Y^{\text {top }} \cup B\right)=Y_{\leq d-2} .
$$

We assume that each $M_{i}$ is oriented and that $M_{i} \cup_{\underline{j \in J_{i}}} B_{j}$ can be given the structure of a manifold with boundary, where $j \in J_{i}$ if the closure $\bar{M}_{i}$ of $M_{i}$ in $Y$ meets $B_{j}$. Similarly, we assume $\overline{B_{j}}-B_{j}$ lies in $Y^{\text {sing }}$. Finally, to get a rational cycle, we assume that $Y$ is labelled. 
This means that the $M_{i}$ have rational labels $\lambda_{i} \in \mathbf{Q}$, assigned so that the following condition holds:

for each $x \in B$, pick an orientation of $T_{x} B$ and divide the components $M_{i}$ that have $x$ in their closure into two groups $I^{+}, I^{-}$according to whether the chosen orientation on $T_{x} B$ agrees with the boundary orientation. Then we require:

$$
\sum_{i \in I^{+}} \lambda_{i}=\sum_{i \in I^{-}} \lambda_{i} .
$$

Note that in many situations when one is considering branching the branching set $B$ is assumed to be closed and so is a codimension 1 submanifold with singularities. Here we have considered these singularities as part of the singular set. An open branched pseudomanifold satisfies all the above conditions except for compactness. One can also consider infinite dimensional branched pseudomanifolds. Here the compactness condition is omitted and the boundary components are cooriented submanifolds of codimension 1 .

Lemma 4.2 Let $Y$ be a (closed, oriented) branched and labelled pseudomanifold of dimension $d$. Then every partially smooth map ev from $Y$ to a closed manifold $X$ defines a rational class ev $v_{*}([Y]) \in H_{d}(X)$.

Proof: Since we are working rationally, the class $e v_{*}([Y])$ is defined by its intersections with classes given by smooth maps $g: Z \rightarrow X$ from oriented closed manifolds $Z$ into $X$. If $\operatorname{dim} Z+d=\operatorname{dim} X$, then we can jiggle $g$ to make it meet each component of the image of $Y_{s m}$ transversally. Hence it will be disjoint from $\operatorname{ev}\left(Y^{\operatorname{sing}} \cup B\right)$ (for reasons of dimension), and will meet $f\left(Y^{\text {top }}\right)$ transversally in a finite number of points. We then set

$$
e v \cdot g=\sum_{i} \varepsilon_{i} \lambda_{i},
$$

where $\varepsilon_{i}= \pm 1$ is the appropriate sign. Observe that if $g_{0}, g_{1}: Z \rightarrow X$ are both transverse to $e v$ in this way, and are joined by a homotopy $G: Z \times[0,1] \rightarrow X$ then we can jiggle $G$ (fixing $g_{i}=\left.G\right|_{Z \times i}$ ) so that it is also transverse to $e v$. The important point now is that the image of $G$ still does not meet $e v\left(Y^{\operatorname{sing}}\right)$ since $Y^{\text {sing }}$ has codimension $\geq 2$ though it may now meet $e v(B)$. It is not hard to check that the condition imposed on the labels in Definition 4.1 guarantees that this number is independent of the jiggling: cf. [S].

In order to define the Gromov-Witten invariants it is sufficient to think of $\overline{\mathcal{M}}^{\nu}$ as a (closed, oriented, partially smooth) branched pseudomanifold. However, in this paper we need a finer structure in which some strata of codimensions 2 and 3 are controlled. (Of course, if necessary, one could control strata of higher codimension.)

Definition 4.3 A (closed, oriented, partially smooth) branched pseudomanifold $Y$ of dimension $d$ is said to have a codimension 2 subcycle $S$ if the following conditions are satisfied:

(i) $S$ is (closed, oriented) branched pseudomanifold of dimension $d-2$;

(ii) There is a neighborhood $\mathcal{N}(S)$ of $S$ in $Y$ and a commutative diagram

$$
\begin{array}{ccc}
\mathcal{N}(S)_{s m} & \rightarrow & \mathcal{N}(S) \\
\pi^{\prime} \downarrow & & \pi \downarrow \\
S_{s m} & \rightarrow & S
\end{array}
$$


where the maps $\pi^{\prime}$ and $\pi$ are oriented locally trivial fibrations with fiber the 2-disc $D^{2}$, where $D_{s m}^{2}=\{0\} \cup\left(D^{2}-\{0\}\right)$. Here the components of $\mathcal{N}(S)_{s m}$ are given by intersecting $\mathcal{N}(S)$ with the components of $Y_{s m}$. In particular this means that the top strata in $S$ are surrounded by top strata in $Y$ that locally look like 2-disc bundles with the zero section deleted. A similar remark applies to the codimension 1 (branching) components.

(iii) It follows from (ii) that the intersection of any top component $M_{i}$ of $Y$ with $\mathcal{N}(S)$ is the pullback of some top component $M_{k}^{S}$ of $S$. We require that $\lambda\left(M_{i}\right)=\lambda\left(M_{k}^{S}\right)$.

Here we have made no attempt to put a smooth structure on the whole of $\pi^{-1}\left(S^{t o p}\right)=$ $\mathcal{N}\left(S^{t o p}\right)$ since, even in the nicest cases, the disc fibers of $\mathcal{N}\left(S^{t o p}\right) \rightarrow S^{t o p}$ may have orbifold singularities at their center: see [Mc2]. The next lemma shows that this is not important.

Proposition 4.4 Let $p: P \rightarrow S^{2}$ be a smooth fibration, and $Y$ be a branched d dimensional pseudomanifold with codimension 2 subcycle $S$. Consider a map ev $: Y \rightarrow P$ such that $S=e v^{-1}(M)$ for some fiber $M$ of $p$. Then if $g: Z \rightarrow M$ is any cycle such that $\operatorname{dim} Z+d=$ $\operatorname{dim} P$

$$
e v_{*}([Y]) \cdot_{P} g_{*}([Z])=e v_{*}([S]) \cdot_{M} g_{*}([Z]) .
$$

Proof: We may jiggle $g: Z \rightarrow M$ so that it meets $e v(S)$ transversally in $M$ in a finite number of points, and hence define $e v_{*}([S]) \cdot_{M} g_{*}([Z])$. The left hand side $e v_{*}([Y]) \cdot_{P} g_{*}([Z])$ is defined by counting intersection points of $e v(Y)$ with a jiggling $g^{\prime}$ of $g$ that meets only the top strata of $Y$. Since $g$ meets only the top strata of $S$, we are locally in the following situation. $g$ is a smooth map $Z \rightarrow\{0\} \times M \subset \mathbf{R}^{2} \times M$ and $e v: D^{2} \times D^{d-2} \rightarrow \mathbf{R}^{2} \times M$ is a continuous map such that

(a) $e v\left(\{0\} \times D^{d-2} \subset\{0\} \times M\right.$;

(b) $e v$ is smooth on $\{0\} \times D^{d-2}$ and on $\left(D^{2}-\{0\}\right) \times D^{d-2}$;

(c) $e v\left(\{0\} \times D^{d-2}\right)$ meets $g(Z)$ transversally in $M$ at the single point $e v(0,0)=\left(0, x_{0}\right)$.

It follows from $(c)$ that $\left.e v\right|_{\{0\} \times D^{d-2}}$ and $g$ are local diffeomorphisms near their intersection point. Clearly we can perturb ev on a small neighborhood of $(0,0)$ in $D^{2} \times D^{d-2}$ so that in addition it is smooth in some neighborhood $U$ of $(0,0)$ and maps the 2-dimensional fibers in $U$ transversally to $M$. It is now clear that we can perturb $g$ near $g^{-1}\left(x_{0}\right)$ to a map $g^{\prime}: Z \rightarrow P$ that meets $e v\left(D^{2} \times D^{d-2}\right)$ exactly once transversally (in $P$ ) and at a point in the image of $e v\left(\left(D^{2}-\{0\}\right) \times D^{d-2}\right)$. The result then follows since by condition (iii) in Definition 4.3 the labellings of $S$ and $Y$ agree.

\subsubsection{Constructing branched pseudomanifolds}

In [Mc3] we show in detail how to construct a branched pseudomanifold from multi-sections of a multi-bundle over a multi-fold. The construction amplifies the method of [LiuT1,2]. Here we will give a very brief sketch of the necessary ingredients.

We start with a space $\mathcal{W}$ in the partially smooth category that locally has the structure of an orbifold. Thus $\mathcal{W}$ is a finite union of open sets $U_{i}, i=1, \ldots, k$, each with uniformizers $\left(\widetilde{U}_{i}, \Gamma_{i}, \pi_{i}\right)$ with the following properties. Each $\Gamma_{i}$ is a finite group acting on $\widetilde{U}_{i}$ and the projection $\pi_{i}$ is the composite of the quotient map $\widetilde{U}_{i} \rightarrow \widetilde{U}_{i} / \Gamma_{i}$ with an identification $\widetilde{U}_{i} / \Gamma_{i}=$ $U_{i}$. The inverse image in $\widetilde{U}_{i}$ of each stratum in $U_{i}$ is an open subset of a (complex) Banach 
space on which $\Gamma_{i}$ acts complex linearly. For simplicity, we suppose that the action of $\Gamma_{i}$ is free on the top strata of $\widetilde{U}_{i}$.

The usual compatibility conditions put on the local uniformizers of an orbifold are replaced by the following construction. For each subset $I=\left\{i_{1}, \ldots, i_{p}\right\}$ of $\{1, \ldots, k\}$ set

$$
U_{I}=\cap_{j \in I} U_{j}, \quad U_{\emptyset}=\emptyset .
$$

Let $\mathcal{N}$ be the set of all $I$ for which $U_{I} \neq \emptyset$. For each $I \in \mathcal{N}$ define the group $\Gamma_{I}$ to be the product $\prod_{j \in I} \Gamma_{j}$ and then define $\widetilde{U}_{I}$ to be the fiber product

$$
\widetilde{U}_{I}=\left\{\tilde{x}_{I}=\left(\tilde{x}_{j}\right)_{j \in I}: \pi_{j}\left(\tilde{x}_{j}\right)=\pi_{\ell}\left(\tilde{x}_{\ell}\right) \in U_{I} \text { for all } j, \ell\right\} \subset \prod_{j \in I} \widetilde{U}_{j},
$$

with its two pullback topologies. Clearly, $\Gamma_{I}$ acts on $\widetilde{U}_{I}$ and the quotient $\widetilde{U}_{I} / \Gamma_{I}$ can be identified with $U_{I}$. If $J \subset I$, there are projections

$$
\pi_{J}^{I}: \widetilde{U}_{I} \rightarrow \widetilde{U}_{J}, \quad \lambda_{J}^{I}: \Gamma_{I} \rightarrow \Gamma_{J},
$$

where $\pi_{J}^{I}$ quotients out by the action of the product group $\Gamma_{I-J}$. We will say that such a collection

$$
\widetilde{\mathcal{U}}=\left\{\left(\widetilde{U}_{I}, \Gamma_{I}, \pi_{J}^{I}, \lambda_{J}^{I}\right): I \in \mathcal{N}\right\}
$$

is a multi-fold atlas $\widetilde{\mathcal{W}}$ for $\mathcal{W}$ or a multi-fold, for short.

The next step is to define a multi-bundle $\tilde{p}: \widetilde{\mathcal{E}} \rightarrow \widetilde{\mathcal{W}}$. Suppose given a space $\mathcal{E}$ that has two topologies, and a map $p: \mathcal{E} \rightarrow \mathcal{W}$ with the property that each set $E_{i}=p^{-1}\left(U_{i}\right)$ has a local uniformizer $\left(\widetilde{E}_{i}, \Gamma_{i}, \pi_{i}\right)$ such that the following diagram commutes:

$$
\begin{array}{cccc}
\widetilde{E}_{i} & \stackrel{\pi_{i}}{\rightarrow} & E_{i} \\
\tilde{p} \downarrow & & \downarrow p \\
\widetilde{U}_{i} & \stackrel{\pi_{i}}{\rightarrow} & U_{i} .
\end{array}
$$

Here we require $\tilde{p}: \widetilde{E}_{i} \rightarrow \widetilde{U}_{i}$ to be a $\Gamma_{i}$-equivariant map that restricts over each stratum of $\widetilde{U}_{i}$ to a locally trivial vector bundle. Thus the fiber $F\left(\tilde{x}_{i}\right)$ of $\tilde{p}$ at each point $\tilde{x}_{i}$ is a vector space, but there is no natural way of identifying one with another if they lie over points in different strata. In the application, the points of $\widetilde{U}_{i}$ are parametrized stable maps $\tilde{\tau}=(\Sigma, \tilde{h})$ and the fiber $F(\tilde{\tau})$ is the space $L^{1, k}\left(\Lambda^{0,1}\left(\Sigma, \tilde{h}^{*} T P\right)\right)$ of sections of a bundle over $\Sigma$. When $\tilde{\tau}$ moves from one stratum to another, the topological type of $\Sigma$ may change, and so there is no easy way to identify these fibers. Thus, $\mathcal{E}$ has the same local structure as $\mathcal{W}$, and we can define a multi-fold atlas $\widetilde{\mathcal{E}}=\left\{\widetilde{E}_{I}\right\}$ for it as above.

Next we define the concept of a multi-section $\tilde{s}$ of a multi-bundle $\widetilde{\mathcal{E}} \rightarrow \widetilde{\mathcal{W}}$. Very roughly speaking this is a compatible collection $\tilde{s}_{I}$ of (sometimes multi-valued) sections of $\widetilde{E}_{I} \rightarrow \widetilde{U}_{I}$ that in the case when $I$ is the singeton $\{j\}$ is simply an ordinary, nonequivariant section of the bundle $\widetilde{E}_{j} \rightarrow \widetilde{U}_{j}$. The whole point of the construction of $\widetilde{\mathcal{E}} \rightarrow \widetilde{\mathcal{W}}$ is that a nonequivariant section $\sigma(j)$ of $\widetilde{E}_{j} \rightarrow \widetilde{U}_{j}$ can be extended to a multi-section $\tilde{s}(j)$ of $\widetilde{\mathcal{E}} \rightarrow \widetilde{\mathcal{W}}$. (Here we are cheating slightly since one has to refine the multi-fold cover $\left\{\widetilde{U}_{I}\right\}$ in order for this to be possible.) A multi-section is said to be Fredholm of index $d$ if (among other conditions) for each $I$ its zero set $\widetilde{Z}_{I}$ is a $d$-dimensional pseudomanifold whose top strata are the intersections with $\widetilde{Z}_{I}$ of the top strata in $\widetilde{U}_{I}$. 
The second main step in the construction is to show how to assemble the zero sets of a Fredholm section $\tilde{s}$ into a branched, labelled pseudomanifold $Y_{\tilde{s}}$. There are two steps. First one replaces the open sets $\widetilde{Z}_{I}$ by compact manifolds with boundary $\widetilde{Y}_{I}$, and then one sets

$$
Y_{\tilde{s}}=\coprod_{I} \tilde{Y}_{I} / \sim
$$

where $\tilde{y}_{I} \sim \tilde{z}_{J}$ if $J \subset I$ and $\pi_{J}^{I}\left(\tilde{y}_{I}\right)=\tilde{z}_{J}$. Elements in the top strata in $Y_{\tilde{s}}$ come from elements in top strata of $\widetilde{U}_{I}$ and their labels derive from the groups $\Gamma_{I}$. For example, points in $Y_{\tilde{s}}^{t o p}$ that are represented in $\widetilde{U}_{j}$ have labels $1 /\left|\Gamma_{j}\right|$. For further details, see [Mc3].

One of the main questions is how to construct Fredholm multi-sections. Below, we will define a finite-dimensional family $\tilde{s}^{\nu}, \nu \in R$, of multi-sections that are Fredholm for generic $\nu$. It follows from the construction that the homology class represented by the corresponding branched pseudomanifold $Y^{\nu}=\overline{\mathcal{M}}^{\nu}$ is independent of all choices.

\subsection{The virtual moduli cycle}

In this subsection we briefly describe how to construct the virtual moduli cycle $\overline{\mathcal{M}}^{\nu}=$ $\overline{\mathcal{M}}_{0, k}^{\nu}(P, J, A)$ as a branched pseudomanifold $Y=Y^{\nu}$ of dimension $d$, where $d=\operatorname{dim} P+$ $2 c_{1}(A)+2 k-6$. Moreover, when $P$ fibers over $S^{2}$ and $A$ is a section class, $Y$ has a codimension 2 subcycle $S$. We use the notation of $\S 2.2 .1$ without further comment and, for simplicity, will often ignore the marked points. In particular, $\mathcal{W}$ will denote a suitable neighborhood of $\overline{\mathcal{M}}$ in the space of all stable maps.

Here are the main features of the structure of the pair $(Y, S)$ when $P$ fibers over $S$ and $A$ is a section class. The elements of $Y$ are parametrized stable maps $(\Sigma, \tilde{h})$ that each satisfy a perturbed Cauchy-Riemann equation

$$
\bar{\partial}_{J} \tilde{h}(w)=\nu_{\tilde{h}}(w), \quad w \in \Sigma,
$$

where $\nu_{\tilde{h}}$ is a $C^{\infty}$-smooth 1 -form in the Sobolev space

$$
\widetilde{L}_{\tilde{h}}=L^{1, p}\left(\Lambda^{0,1}\left(\Sigma, \tilde{h}^{*}\left(T_{\text {vert }} P\right)\right)\right)
$$

consisting of all sections of the bundle $\Lambda^{0,1}\left(\Sigma, \tilde{h}^{*}\left(T_{\text {vert }} P\right)\right)$ that are $L^{1, p}$-smooth on each component. (Here $T_{\text {vert }} P$ denotes the tangent bundle to the fibers of $P \rightarrow S^{2}$.) There is a finite-to-one map $\pi: Y \rightarrow \mathcal{W}$ that forgets the parametrization, such that each stratum in $Y_{s m}$ of codimension $2 k$ or $2 k+1$ is taken to a stratum in $\mathcal{W}$ consisting of elements whose domain has $\leq k+1$ components. (We would have equality here if it were not for the extra strata that are introduced to deal with the singular points of the closure of the branching locus.) In particular, the top strata $M_{i}$ and the branch components $B_{j}$ consist of maps with domain a single sphere. Further the codimension 2 subcycle $S$ consists of all elements whose domain contains more than one sphere.

The evaluation map ev: $\overline{\mathcal{M}}^{\nu} \rightarrow P^{k}$ is given by composing the forgetful map $\pi: Y=$ $\overline{\mathcal{M}}^{\nu} \rightarrow \mathcal{W}$ with the evaluation map $\mathcal{W} \rightarrow P^{k} . \overline{\mathcal{M}}^{\nu}$ is called a virtual moduli cycle because the class represented by ev $: \overline{\mathcal{M}}^{\nu} \rightarrow P^{k}$ can be used to calculate Gromov-Witten invariants. Presumably it is the same as the class $e v_{*}\left(\mathcal{C}_{0, k}^{\text {hol }}\right)$ considered in $\S 2$, but it is not clear whether anyone has yet checked this. Therefore from now on we will define Gromov-Witten invariants using $\overline{\mathcal{M}}^{\nu}$. Sometimes we will call $\overline{\mathcal{M}}^{\nu}$ a regularization of $\overline{\mathcal{M}}$. 


\subsubsection{The local construction}

The first step is to show that locally $\mathcal{W}$ has the structure assumed in $\S 4.1$. More precisely, Liu-Tian show in [LiuT1] Lemma 2.6 that each $\tau \in \overline{\mathcal{M}}$ has an open neighborhood $U_{\tau}$ of the form $\widetilde{U}_{\tau} / \Gamma_{\tau}$, where the elements of $\widetilde{U}_{\tau}$ are parametrized stable maps $\tilde{\tau}=(\Sigma, \tilde{h})$ and $\Gamma_{\tau}$ acts via reparametrizations. Thus this local uniformization $\left(\widetilde{U}_{\tau}, \Gamma_{\tau}\right)$ is given by a constructing a local $\Gamma_{\tau}$-equivariant slice for the action of the reparametrization group on the space of parametrized stable maps.

Several comments are in order here. Firstly, one usually wants to consider orbifolds that are modelled on Banach manifolds. Thus, best of all would be to have $\widetilde{U}_{\tau}$ an open subset of a Banach space with a linear action of $\Gamma_{\tau}$. (It is possible, but not easy, to arrange this: see Siebert $[\mathrm{Sb}]$. .) Instead of this, Liu-Tian show that $\widetilde{U}_{\tau}$ is partially smooth.

A second point is that in order to construct their slice Liu-Tian fix the images by $h$ of certain added marked points on $\Sigma$, which entails the use of the $L^{2, p}$-topology on all spaces of maps with domain of fixed topological type. This makes gluing more complicated. However, one can avoid this complication (and instead use the $L^{1, p}$-topology on the strata in $\mathcal{W}$ ) by Siebert's clever idea of using integral conditions to fix the parametrization: see [Sb]. With this approach, certain details of the argument below would have to be changed.

In order to define the overall topology on $\mathcal{W}$ one must understand how the topologies on the different strata fit together. The neighborhood $U_{\tau}$ is chosen so that all domains $\Sigma_{\tau^{\prime}}$ that occur form a $2 k$-dimensional family (where $\Sigma_{\tau}$ has $k+1$ components) much as the fibers of the projection $\mathcal{S} \rightarrow \Delta$ described in $\S 2.3 .2$. The parameters in this family are called "gluing parameters": their role is briefly described in the discussion just before Lemma 4.5 below. Once one knows how to topologize the set of domains, it is not hard to say what is meant by nearby stable maps. As a point of notation, we write $\tau=[\Sigma, h]$ for elements of $\mathcal{W}$ and $\tilde{\tau}=(\Sigma, \tilde{h})$ for their parametrized lifts.

The next step is to define a local bundle $\widetilde{\mathcal{L}}_{\tau} \rightarrow \widetilde{U}_{\tau}$ whose fiber at an element $\tilde{\tau}^{\prime}=\left(\Sigma^{\prime}, \tilde{h}^{\prime}\right)$ is the space $\widetilde{L}_{\tilde{h}^{\prime}}$ defined above. Note that the fiber changes as when the topological type of the domain $\Sigma^{\prime}$ changes. But it is a locally trivial bundle over each stratum and it is not hard to give it a global topology. Therefore it is a bundle in our category. Clearly, the action of $\Gamma_{\tau}$ lifts to $\widetilde{\mathcal{L}}_{\tilde{h}}$.

The Cauchy-Riemann operator $\bar{\partial}_{J}$ gives rise to a $\Gamma_{\tau}$-equivariant section of $\widetilde{\mathcal{L}}_{\tau} \rightarrow \widetilde{U}_{\tau}$. Moreover, its linearization at $\tilde{\tau}^{\prime}$ is:10

$$
D \tilde{h}^{\prime}: L^{2, p}\left(\Sigma^{\prime},\left(\tilde{h}^{\prime}\right)^{*}(T P)\right) \rightarrow \widetilde{L}_{\tilde{h}^{\prime}},
$$

where $p>2$. Now look at the "center" point $\tau$ of $U_{\tau}$ and let $\tilde{\tau}=(\Sigma, \tilde{h}) \in \widetilde{U}_{\tau}$ be one of its lifts. Since $D \tilde{h}$ is elliptic, there is a finite dimensional subspace $R_{\tau}$ of the vector space $C^{\infty}\left(\Lambda^{0,1}\left(\Sigma \times P, p r^{*} T P\right)\right)$ such that the operator

$$
D \tilde{h} \oplus e: L^{2, p}\left(\Sigma, \tilde{h}^{*}(T P)\right) \oplus R_{\tau} \rightarrow \widetilde{L}_{\tilde{h}}
$$

is surjective, where $e$ is given by restricting the sections in $R_{\tau}$ to the graph of $\tilde{h}$.

\footnotetext{
${ }^{10}$ If one used Siebert's way of fixing parametrizations one could consider this as a map from $L^{1, p}$-sections to $L^{p}$-sections. Observe also the domain should be cut down by a finite number of conditions to fix the parametrizations of the stable maps. This makes no essential difference to the present argument, and will be glossed over here. A precise definition of the domain of $D \tilde{h}$ is given in the proof of Lemma 4.9 below.
} 
The next step (see [LiuT1] §3) is to show that we can choose $R_{\tau}$ and a partially smooth family of embeddings $e_{\tilde{\tau}^{\prime}}: R_{\tau} \rightarrow \widetilde{L}_{\tilde{h}^{\prime}}$, so that

$$
D \tilde{h}_{\tau^{\prime}} \oplus e_{\tilde{\tau}^{\prime}}: L^{2, p}\left(\tilde{h}_{\tau^{\prime}}^{*}(T P)\right) \oplus R_{\tau} \rightarrow \widetilde{L}_{\tilde{h}^{\prime}}
$$

is surjective (with uniform estimates for the inverse) for all $\tilde{\tau}^{\prime} \in \widetilde{U}_{\tau}$. Here we are allowed to shrink $U_{\tau}$. The fact that this is possible is a deep result. If $\tilde{\tau}^{\prime}$ lies in the same stratum as $\tilde{\tau}$ it holds because of the openness of the regularity condition. However, to prove this in general one has to show that there is a uniformly bounded family of right inverses to $D \tilde{h}_{\tau^{\prime}} \oplus e_{\tilde{\tau}^{\prime}}$ as $\tilde{\tau}^{\prime}$ varies over a small enough neighborhood $\widetilde{U}_{\tau}$. This analytic fact is the basis of all gluing arguments.

With this done, let $p r: \widetilde{U}_{\tau} \times R_{\tau} \rightarrow \widetilde{U}_{\tau}$ denote the projection and consider the pullback bundle $\operatorname{pr}^{*}\left(\widetilde{\mathcal{L}}_{\tau}\right) \rightarrow \widetilde{U}_{\tau} \times R_{\tau}$. This bundle has a section $s$ defined by

$$
s\left(\tilde{\tau}^{\prime}, \nu\right)=\bar{\partial}_{J}\left(\tilde{h}_{\tau^{\prime}}\right)+e_{\tilde{\tau}^{\prime}}(\nu) .
$$

By construction, its linearization is surjective at all points $(\tilde{\tau}, 0)$ and so it remains surjective for $|\nu| \leq \varepsilon$. One then shows by some variant of gluing that the intersection of $s$ with the zero section is a (partially smooth) open pseudomanifold of dimension equal to ind $D \tilde{h}+\operatorname{dim} R_{\tau}$, whose components are given by intersecting the solution space with the different strata in $\widetilde{U}_{\tau}$. By considering the projection of this solution space to $R_{\tau}$, one sees that the set of solutions for fixed generic $\nu \in R_{\tau}$ is still an open pseudomanifold that we will call $\widetilde{Z}_{\tau}^{\nu}$. Since $\widetilde{Z}_{\tau}^{\nu} \subset \widetilde{U}_{\tau}$, there is a forgetful map $\pi: \widetilde{Z}_{\tau}^{\nu} \rightarrow \mathcal{W}$.

This pseudomanifold $\widetilde{Z}_{\tau}^{\nu}$ has one other very important property that we will need in order to establish the fact that the virtual moduli cycle $Y$ has a codimension 2 subcycle. Namely, if the domain of $\tilde{\tau}^{\prime} \in \widetilde{Z}_{\tau}^{\nu}$ has $k+1$ components then the nearby elements of $\widetilde{Z}_{\tau}^{\nu}$ may be obtained from $\tilde{\tau}^{\prime}$ by gluing. When $k=1$ (the case of interest to us) this means the following. Let $\mathcal{W}_{2}$ denote the subset of $\mathcal{W}$ consisting of elements $\tau$ whose domain has $\geq 2$ components, and suppose that $\widetilde{\mathcal{S}}$ is a stratum of $\widetilde{Z}_{\tau}^{\nu}$ that is taken by $\pi$ into a top stratum of $\mathcal{W}_{2}$. Then the domain $\Sigma_{\tau^{\prime}}$ for $\tau^{\prime} \in \widetilde{\mathcal{S}}$ has 2 components $\Sigma_{1} \cup \Sigma_{2}$ intersecting at $x=\Sigma_{1} \cap \Sigma_{2}$. Hence there is a line bundle $V \rightarrow \widetilde{\mathcal{S}}$ whose fiber at $\tilde{\tau}^{\prime}$ is $T_{x}\left(\Sigma_{1}\right) \otimes T_{x}\left(\Sigma_{2}\right)$. Each sufficiently small element $a$ of the fiber of $V$ at $\tilde{\tau}^{\prime}$ serves as a gluing parameter, in the sense that there is a recipe for making a single sphere $\Sigma_{a}$ from the union $\Sigma_{1} \cup \Sigma_{2}$ and for constructing a map $g\left(\tilde{\tau}^{\prime}, a\right): \Sigma_{a} \rightarrow P$. Moreover $\left(\Sigma_{a}, g\left(\tilde{\tau}^{\prime}, a\right)\right) \in \widetilde{Z}_{\tau}^{\nu}$ and the map

$$
\left(\tilde{\tau}^{\prime}, a\right) \mapsto\left(\Sigma_{a}, g\left(\tilde{\tau}^{\prime}, a\right)\right)
$$

is a bijection from a neighborhood of $\widetilde{\mathcal{S}}$ in $V$ to a neighborhood of $\widetilde{\mathcal{S}}$ in $\widetilde{Z}_{\tau}^{\nu}$. (See [LiuT1] Proposition 3.2 or $[\mathrm{FO}]$.)

The above discussion shows:

Lemma 4.5 The local solution set $\widetilde{Z}_{\tau}^{\nu}$ has the structure of an open branched pseudomanifold with codimension 2 subcycle $S=\widetilde{Z}_{\tau}^{\nu} \cap \pi^{-1}\left(\mathcal{W}_{2}\right)$.

\subsubsection{The global construction}

Because $\overline{\mathcal{M}}$ is compact, it is covered by a finite number $U_{j}, j=1, \ldots, k$ of the sets $U_{\tau}$ and $\mathcal{W}$ is defined to be their union. We write $\left(\widetilde{U}_{j}, \Gamma_{j}, \pi_{j}\right)$ for the corresponding uniformizers, 
where

$$
\pi_{j}: \widetilde{U}_{j} \rightarrow \widetilde{U}_{j} / \Gamma_{j}=U_{j}
$$

is the obvious projection. Let $\widetilde{\mathcal{L}} \rightarrow \widetilde{\mathcal{W}}$ denote the multi-bundle that restricts on $\widetilde{U}_{j}$ to $\widetilde{\mathcal{L}}_{j} \rightarrow \widetilde{U}_{j}$. The Cauchy-Riemann operator $\bar{\partial}_{J}$, being equivariant, defines a multi-section of $\widetilde{\mathcal{L}} \rightarrow \widetilde{\mathcal{W}}$, and the problem is to define a suitable family of global perturbations.

To this end, choose a partition of unity $\beta_{j}$ on $\mathcal{W}$ subordinate to the covering $\left\{U_{j}\right\}$ and define the section $\sigma(j)$ of the pullback bundle $\operatorname{pr}^{*}\left(\widetilde{\mathcal{L}}_{j}\right) \rightarrow \widetilde{U}_{j} \times R_{j}$ by

$$
\sigma(j)\left(\tilde{\tau}^{\prime}, \nu_{j}\right)=\beta_{j}(\tau) \cdot e_{\tilde{\tau}^{\prime}}\left(\nu_{j}\right) .
$$

Choose $\varepsilon>0$ so that, for all $j$, the linearization of $\sigma(j)$ is surjective at all points $\left|\nu_{j}\right| \leq \varepsilon$, and put

$$
R=\oplus_{j} R_{j}, \quad R_{\varepsilon}=\left\{\nu=\left(\nu_{j}\right) \in R:\left|\nu_{j}\right| \leq \varepsilon, \text { for all } j .\right\}
$$

Then set $\mathcal{W}_{\varepsilon}=\mathcal{W} \times R_{\varepsilon}$. It is shown in [Mc3] that the pull back multi-bundle $\operatorname{pr}^{*}(\widetilde{\mathcal{L}}) \rightarrow \widetilde{\mathcal{W}}_{\varepsilon}$ has a Fredholm multi-section $\tilde{s}$ that is the sum of $\bar{\partial}_{J}$ with a finite number of sections $\tilde{s}(j)$, where $\tilde{s}(j)$ restricts over $\widetilde{U}_{j}$ to $\beta_{j} \sigma(j)$. Hence the corresponding multi-section $\tilde{s}^{\nu}$ of $\widetilde{\mathcal{L}} \rightarrow \widetilde{\mathcal{W}}$ is Fredholm for generic $\nu \in R$.

Proposition 4.6 For generic $\nu \in R$ the zero sets $\widetilde{Z}_{I}^{\nu}$ of the multi-section $\tilde{s}^{\nu}$ of $\widetilde{\mathcal{L}} \rightarrow \widetilde{\mathcal{W}}$ fit together to give a branched pseudomanifold $\overline{\mathcal{M}}^{\nu}$ of dimension $d$ and with codimension 2 subcycle.

Proof: The proof is sketched above except for the statement about the codimension 2 subcycle. Let $\mathcal{W}_{2}$ denote the set of all elements in $\mathcal{W}$ whose domain has $>1$ component, and choose the covering $U_{j}, j=1, \ldots, k$, of $\overline{\mathcal{M}}$ so that its first $\ell$ elements have centers $\tau \in \mathcal{W}_{2}$ and so that the set $\cup_{j>\ell} U_{j}$ is disjoint from some neighborhood $\mathcal{N}_{2}$ of $\overline{\mathcal{M}} \cap \mathcal{W}_{2}$. It follows from Lemma 4.5 that the local solution set $\widetilde{Z}_{I}^{\nu}$ of $\tilde{s}^{\nu}$ has a codimension 2 subcycle

$$
\widetilde{S}_{I}=\widetilde{Z}_{I}^{\nu} \cap \pi^{-1}\left(\mathcal{W}_{2}\right)
$$

for $I \subset\{1, \ldots, \ell\}$, and for other $I$ does not meet $\pi^{-1}\left(\mathcal{W}_{2}\right)$. Therefore, we just have to see that in the process of forming the pseudocycle $Y$ these local subcycles fit together to form a global subcycle $S$. The identifications needed to make $Y$ come from the combinatorics of two subcovers, one a shrinking $\left\{U_{j}^{0}\right\}$ of $\left\{U_{j}\right\}$ and another associated subcover $\left\{V_{I}\right\}$ of $\left\{U_{I}\right\}$ : see [LiuT1] Lemma 4.3 and [Mc3] Lemma 4.9. It follows from the construction detailed in [Mc3] 44.3 that all we need in order for the subcycle $S$ to exist is that these subcovers are chosen to be products near $\mathcal{W}_{2}$. Thus, if $r: \mathcal{N}_{2} \rightarrow \mathcal{W}_{2}$ is a retraction, it suffices that

$$
V_{I}=r^{-1}\left(V_{I} \cap \mathcal{W}_{2}\right), \quad U_{j}^{0}=r^{-1}\left(U_{j}^{0} \cap \mathcal{W}_{2}\right),
$$

for $j, I \subset\{1, \ldots, \ell\}$. Since it is possible to define the $V_{I}, U_{j}^{0}$ in this way, the result follows. $\square$

Definition 4.7 Any $d$ dimensional branched and labelled pseudomanifold $Y=\overline{\mathcal{M}}^{\nu}$ constructed as above from some covering of a neighborhood $\mathcal{W}$ of the space $\overline{\mathcal{M}}_{0, k}(P, J, A)$ will be called a regularization of $\overline{\mathcal{M}}_{0, k}(P, J, A)$. 
By considering a 1-parameter version of this construction, and by considering what happens under a refinement of the covers, Liu-Tian show that, for any regularization $\overline{\mathcal{M}}^{\nu}$ of $\overline{\mathcal{M}}_{0, k}(P, J, A)$, the rational bordism class of the map ev $: \overline{\mathcal{M}}^{\nu} \rightarrow P^{k}$ is independent of the choice of $J$, of coverings $\left\{U_{j}\right\}$, and of all other auxiliary structures. It depends only on the deformation class of of $\Omega$ on $P$, on the homology class $A$ and on the number of marked points $k$.

\subsection{Properties of Gromov-Witten invariants}

In this subsection we prove Proposition 1.6 and complete the arguments started in $\S 2.4$. This will complete the proof of Theorem 1.1, and hence of all our other results. We begin by describing the special properties of Gromov-Witten invariants in fibered spaces.

\subsubsection{Gromov-Witten invariants in $P \rightarrow S^{2}$}

The following result is immediate from the construction given in $§ 4.2$.

Proposition 4.8 Suppose that $V$ is a subbundle of TP such that for all representatives $\tilde{\tau}=(\Sigma, \tilde{h})$ of the elements $\tau \in \overline{\mathcal{M}}$ the cokernel of D $\tilde{h}$ is spanned by elements of the space

$$
\mathcal{L}_{\tilde{h}}^{V}=L^{1, p}\left(\Lambda^{0,1}\left(\tilde{h}^{*}(V)\right)\right)
$$

Then we can choose $R$ and the embeddings e so that for all $\nu \in R$ and all $\tilde{\tau}$

$$
e_{\tilde{\tau}}(\nu) \in \mathcal{L}_{\tilde{h}}^{V}
$$

We now consider the case when $P=P_{\phi}$ fibers over $S^{2}$. Recall from Definition 2.8 what it means for $J$ to be compatible with this fibration.

Lemma 4.9 Let $P \rightarrow S^{2}$ be a symplectic fibration with compatible almost complex structure $J$, and suppose that $A \in H_{2}(P)$ is either a section or a fiber class. Then we can take $V$ in the above proposition to be the vertical tangent bundle $T_{\text {vert }} P$.

Proof: By Lemma 2.9, if $J$ is fibered every component $C_{i}=\tilde{h}\left(\Sigma_{i}\right)$ of the image of a $J$-holomorphic stable map is either contained in a single fiber, or is a section. If $C_{i}$ is a section, the bundle $\tilde{h}_{i}^{*}(T P)$ splits as the direct sum $\tilde{h}_{i}^{*}\left(T C_{i}\right) \oplus \tilde{h}_{i}^{*}\left(T_{\text {vert }} P\right)$. Correspondingly

$$
L^{1, p}\left(\Lambda^{0,1}\left(\tilde{h}_{i}^{*}(T P)\right)\right)=L^{1, p}\left(\Lambda^{0,1}\left(\tilde{h}_{i}^{*}\left(T C_{i}\right)\right)\right) \oplus L^{1, p}\left(\Lambda^{0,1}\left(\tilde{h}_{i}^{*}\left(T_{\text {vert }} P\right)\right)\right) .
$$

Direct calculation shows that $D \tilde{h}_{i}$ maps $L^{2, p}\left(\tilde{h}_{i}^{*}\left(T C_{i}\right)\right)$ onto $L^{1, p}\left(\Lambda^{0,1}\left(\tilde{h}_{i}^{*}\left(T C_{i}\right)\right)\right)$. If $C_{i}$ lies in the fiber, then $\tilde{h}_{i}^{*}(T P)$ splits as $\mathbf{C} \oplus \tilde{h}_{i}^{*}\left(T_{\text {vert }} P\right)$, where $\mathbf{C}$ denotes the trivial bundle. In this case also, $D \tilde{h}_{i}$ maps $L^{2, p}(\mathbf{C})$ onto $L^{1, p}\left(\Lambda^{0,1}(\mathbf{C})\right)$ because the domain of $\tilde{h}_{i}$ is a sphere.

Thus the result holds for each component separately. To deal with $\Sigma$ as a whole, we must develop some notation to describe its intersection pattern. Because the components of $\Sigma$ are attached according to a tree graph it is easy to see that we can order them, keeping $\Sigma_{0}$ as the stem, so that the union $\Sigma_{0} \cup \ldots \cup \Sigma_{i}$ is connected for each $i$. Thus each $\Sigma_{i}$ for 
$i>0$ is attached to a unique component $\Sigma_{j_{i}}$ for $j_{i}<i$. Hence there is a point $x_{i} \in \Sigma_{i}$ that is identified with $y_{j_{i}} \in \Sigma_{j_{i}}$. Then the domain of the map $D \tilde{h}$ is

$$
L^{2, p}\left(\tilde{h}^{*}(T P)\right)=\left\{\oplus_{i} \xi_{i} \in \oplus_{i} L^{2, p}\left(\tilde{h}_{i}^{*}(T P)\right): \xi_{i}\left(x_{i}\right)=\xi_{j_{i}}\left(y_{j_{i}}\right) 1 \leq i \leq \ell\right\},
$$

and its range is

$$
\oplus_{i=0}^{\ell} L^{1, p}\left(\Lambda^{0,1}\left(\tilde{h}_{i}^{*}(T P)\right)\right) .
$$

Some remarks are needed here. Note first that the elements of $L^{2, p}\left(\tilde{h}^{*}(T P)\right)$ satisfy compatibility conditions at the double points since the image of a stable map is always connected. Secondly, in order to fix the parametrization of the maps near $\tilde{h}$ (i.e. in order to construct the local slice $\widetilde{U}_{\tau}$ ), one does not look at all maps near $\tilde{h}$ but only at those that satisfy certain extra normalization conditions. To do this, Liu-Tian add a minimum number of marked points $w_{i}$ to $\Sigma$ to make each component of the curve $\Sigma$ stable, i.e. so that each component has at least 3 special points. Then for each $w_{i}$ they choose a hypersurface $\mathbf{H}_{i}$ in $P$ that meets $\operatorname{Im} \tilde{h}$ transversally at $\tilde{h}\left(w_{i}\right)$ and consider only those maps $\tilde{h}^{\prime}: \tilde{h}^{\prime}\left(w_{i}\right) \in \mathbf{H}_{i}$ : see [LiuT1] §2. We can choose $\mathbf{H}_{i}$ to be the fiber through $\tilde{h}\left(w_{i}\right)$ if $w_{i}$ is on the stem and so that the horizontal direction is contained in its tangent space at $\tilde{h}\left(w_{i}\right)$ if $w_{i}$ is on a branch. The domain of $D \tilde{h}$ is then cut down by the corresponding restrictions. Thus, if there is an added point $w_{i}$ on the stem then $\xi_{0}\left(w_{i}\right)$ must be vertical, and if $w_{i}$ is on the branch component $\Sigma_{j}$ there is a restriction on the vertical (but not the horizontal) part of $\xi_{j}\left(w_{i}\right)$. Let us denote this restricted domain by $\operatorname{Dom}_{r e s}(D \tilde{h})$.

In order to prove the lemma we need to see that the "horizontal part" of the range

$$
V_{h o r}=L^{1, p}\left(\Lambda^{0,1}\left(\tilde{h}_{0}^{*}\left(T C_{0}\right)\right)\right) \oplus\left(\oplus_{i=1}^{\ell} L^{1, p}\left(\Lambda^{0,1}(\mathbf{C})\right)\right)
$$

is in the image by $D \tilde{h}$ of the restricted domain $\operatorname{Dom}_{r e s}(D \tilde{h})$. It follows from what we have already proved that, for every element $v=\left(v_{0}, \ldots, v_{\ell}\right)$ of $V_{h o r}$, there is $\xi=\left(\xi_{0}, \ldots, \xi_{\ell}\right)$ such that $v_{i}=D \tilde{h}_{i}\left(\xi_{i}\right)$. (The reader can check that the extra restrictions put on the $\xi_{i}$ by the $w_{i}$ make no difference here.) Moreover, we can take $\xi_{i} \in L^{2, p}(\mathbf{C})$ for all $i>0$. Thus, when $i>0, \xi_{i}$ is simply a function on $\Sigma_{i}$ and we can alter it by a constant without changing its image under $D \tilde{h}_{i}$. Hence, if we define $\xi^{\prime}=\left(\xi_{0}^{\prime}, \ldots, \xi_{\ell}^{\prime}\right)$ inductively by setting

$$
\xi_{0}^{\prime}=\xi_{0}, \quad \xi_{i}^{\prime}=\xi_{i}+\xi_{j_{i}}\left(y_{j_{i}}\right)-\xi_{i}\left(x_{i}\right),
$$

we will have

$$
\xi^{\prime} \in \operatorname{Dom}_{r e s}(D \tilde{h}), \quad D \tilde{h}\left(\xi^{\prime}\right)=D h(\xi)=v .
$$

Hence result.

Definition 4.10 The pair $(J, \nu)$ on $P$ is said to be fibered if $J$ is compatible with the fibration (as described in Definition 2.8) and if $\nu$ is made as above from sections of the vertical tangent bundle $T_{\text {vert }} P$.

The previous two results imply:

Corollary 4.11 If $A$ is a fiber or section class in $P_{\phi}$ it is possible to construct the regularized moduli cycle $\overline{\mathcal{M}}^{\nu}=\overline{\mathcal{M}}_{0,2}^{\nu}\left(P_{\phi}, J, A\right)$ using a fibered pair $(J, \nu)$. 
Proposition 4.12 If $(J, \nu)$ is fibered and $\sigma$ is a section class, then every element $\tau \in \overline{\mathcal{M}}^{\nu}=$ $\overline{\mathcal{M}}_{0,2}^{\nu}\left(P_{\phi}, J, \sigma\right)$ has a stem-branch structure. In other words, one component is a section of $P_{\phi}$ and all the others lie in fibers. The top strata in $\overline{\mathcal{M}}^{\nu}$ have dimension $d=2 n+4+2 c_{\phi}(\sigma)$, and consist of sections of $P$. The codimension 2 strata consists of elements with precisely two components, the stem and a branch consisting of one bubble.

Proof: Each component $h_{i}$ of $h$ satisfies an equation of the form

$$
\bar{\partial}_{J} h_{i}=\nu_{i}
$$

where $\nu_{i}$ is a section of $\Lambda^{0,1}\left(h_{i}^{*}\left(T_{\text {vert }} P_{\phi}\right)\right)$. Hence the map $\pi \circ h_{i}: S^{2} \rightarrow S^{2}$ is holomorphic and the first statement follows as in Lemma 2.9. The statement about the strata follow firstly from the index formulas in (5) in $\S 2.2 .1$, and secondly from the arguments in Proposition 4.6.

Remark 4.13 Suppose that $X \rightarrow B$ is a fibration with fiber $M$, compact base manifold $B$, and structural group $\operatorname{Symp}(M, \omega)$. Then, if $A \in H_{2}(X, \mathbf{Z})$ is a fiber class (i.e. is in the image of $\left.\iota: H_{2}(M) \rightarrow H_{2}(X)\right)$, and if $J$ is an almost complex structure on the vertical tangent bundle $T_{\text {vert }} X$ that is tamed by the symplectic forms on the fibers, the above arguments show that we can regularize the moduli space $\overline{\mathcal{M}}_{0, k}(X, J, B)$ by a fiberwise perturbation term $\nu$. The resulting virtual moduli cycle $\overline{\mathcal{M}}_{0, k}^{\nu}$ is exactly what is needed to define the fiberwise Gromov-Witten invariants considered by Le-Ono in [LO] and Seidel in [S2]. Note in particular that, as in Proposition 4.12, each element in $\overline{\mathcal{M}}_{0, k}^{\nu}$ has image in a single fiber.

\subsubsection{The Gromov-Witten invariant $n_{P}(\iota(a), \iota(b) ; \sigma)$}

The Gromov-Witten invariant $n_{P}(\iota(a), \iota(b) ; \sigma)$ is the intersection number of the cycle

$$
e v\left(\overline{\mathcal{M}}_{0,2}^{\nu}\left(P_{\phi}, J, \sigma\right)\right)
$$

in $P_{\phi} \times P_{\phi}$ with a generic representative of the class $\iota(a \times b)$, where $\iota$ is the obvious inclusion and the dimensions are such that transverse intersections are isolated. In what follows we will always assume that the pair $(J, \nu)$ is fibered, and that the dimension condition

$$
\operatorname{dim}(a)+\operatorname{dim}(b)+2 c_{\phi}(\sigma)=2 n
$$

is satisfied. The next lemma shows that we can achieve transversality by representing $\iota(a \times b)$ by a cycle of the form $\alpha \times \beta$ where $\alpha, \beta$ lie in (distinct) fibers of $P_{\phi}$.

Lemma 4.14 If $(J, \nu)$ is fibered, then when evaluating $n_{P}(\iota(a), \iota(b) ; \sigma)$ we may assume that the representative of the class $\iota(a \times b)$ has the form $\alpha \times \beta$ where $\alpha, \beta$ are cycles that represent $\iota(a)$ and $\iota(b)$ respectively and lie in distinct fibers of $P_{\phi}$. Further all intersection points are transverse and occur with elements in the top stratum.

Proof: Let $Y=\overline{\mathcal{M}}_{0,2}^{\nu}\left(P_{\phi}, J, \sigma\right)$ where $(J, \nu)$ is fibered. Choose generic representatives $\alpha, \beta$ of the classes $\iota(a), \iota(b)$ that lie in distinct generic fibers. As before, since we are working with rational homology we may assume that $\alpha, \beta$ are the images of smooth closed manifolds. We have to show that all the intersections of $\alpha \times \beta$ with $e v\left(Y^{\text {top }}\right)$ are transverse in $P_{\phi} \times P_{\phi}$ and 
that $\alpha \times \beta$ meets no other elements in the image of $e v$. This would be obvious for dimensional reasons if it were not for the fact that we allow only a restricted class of perturbations of $\alpha, \beta$.

First consider the composite

$$
(\pi \times \pi) \circ e v \circ i_{Y}: Y_{s m} \rightarrow Y \rightarrow P \times P \rightarrow S^{2} \times S^{2} .
$$

It is smooth, and so we may choose a regular value $(\bar{x}, \bar{y}) \in S^{2} \times S^{2}$. Now let $\alpha$ (resp. $\beta$ ) be a generic representative of $\iota(a)$ in $\pi^{-1}(\bar{x})$, (resp. of $\iota(b)$ in $\pi^{-1}(\bar{y})$. Consider an intersection point $(x, y)$ of $e v \circ i_{Y}\left(Y_{s m}\right)$ with $\alpha \times \beta$, and let $V \subset T_{(x, y)} P_{\phi} \times P_{\phi}$ be the span of the tangent spaces to $\alpha, \beta$ and $\left(e v \circ i_{Y}\right)_{*}\left(T Y_{s m}\right)$. Since $V$ projects onto $T_{(\pi(x), \pi(y))} S^{2} \times S^{2}$ by hypothesis, the intersection of $V$ with $T_{\text {vert }} P \times T_{\text {vert }} P$ has dimension at most $4 n$, with equality only if the dimension of $\left(e v \circ i_{Y}\right)_{*}\left(T Y_{s m}\right)$ is maximal, i.e. if the intersection is with a point in $e v\left(Y^{t o p}-B\right)$. But if the dimension is $<4 n$ we could get rid of this intersection point by perturbing $\alpha$ and $\beta$ in their respective fibers. Since we assumed that $\alpha, \beta$ were generic under such perturbations, this is not possible. Hence $(x, y)$ is a transversal intersection point, and lies in $e v\left(Y^{\text {top }}\right)$ as claimed.

\section{Proof of Proposition 1.6}

Part (i) follows as at the end of $\S 2.2 .2$. (Note that the argument given there is no longer applicable, since it is not clear whether Siebert's invariants coincide with the ones we are now considering.) Part (ii) states that

$$
n_{P}(a, v, w ; B)=n_{M}(a, v \cap[M], w \cap[M] ; B) .
$$

Choose representatives $\alpha, \tilde{\beta}, \tilde{\gamma}$ for $a, v, w$ so that $\alpha$ lies in a fiber $F$ of $P$ that intersects $\tilde{\beta}$ and $\tilde{\gamma}$ transversally. Let $(J, \nu)$ be fibered, and consider the intersection

$$
e v_{*}\left(\overline{\mathcal{M}}_{0,3}^{\nu}(P, J, B)\right) \cap(\alpha \times \tilde{\beta} \times \tilde{\gamma}) \subset P^{3} .
$$

Since each element of $\overline{\mathcal{M}}_{0,3}^{\nu}(P, J, B)$ is a stable map with image in a single fiber of $P$, the only curves that concern us are those lying in $F$. Clearly, by arguing as in Lemma 4.14 above, one can make the intersection transverse by perturbing $\alpha$ so that it always remains in some fiber that is transver to $\tilde{\beta}, \tilde{\gamma}$. Further, it is clear from the construction of the virtual moduli cycle described in $\S 4.1-3$ that the subset of $\overline{\mathcal{M}}_{0,3}^{\nu}(P, J, B)$ consisting of stable maps with image in $F$ regularizes the moduli space of $B$-curves in $M$ in the sense of Definition 4.7. Hence there is a bijective correspondence between the intersection points that contribute to $n_{P}(a, v, w ; B)$ and those that contribute to $n_{M}(a, v \cap[M], w \cap[M] ; B)$. Since the orientations also correspond, the result follows.

Part (iii) states that for all $v \in H_{*}(P), v \neq[P]$

$$
n_{P}(a, b, v \cap[M],[M] ; \sigma)=n_{P, \chi}(a, b, v,[M] ; \sigma) .
$$

To prove this, construct $\overline{\mathcal{M}}_{4}^{\nu}=\overline{\mathcal{M}}_{0,4}^{\nu}(P, J, \sigma)$ as usual, choose $t$ to be a regular value of the cross ratio $\mathrm{cr}: \overline{\mathcal{M}}_{4}^{\nu} \rightarrow S^{2}$. and set

$$
\overline{\mathcal{M}}_{4, t}^{\nu}=c r^{-1}\left(\chi_{0}\right) .
$$


As in Lemma 4.14, one can make the cycle $\alpha \times \beta \times \nu \times M_{0}$ transverse to the image of $\overline{\mathcal{M}}_{4, t}$ by perturbing $\alpha$ and $\beta$ within given (generic) fibers $F_{0}, F_{1}$ of $P$, by taking $M_{0}$ to be a generic fiber $F_{\infty}$ and by perturbing $\nu$ within the class of cycles that are products near the fiber $F_{z}$ at the point $z$ corresponding to the given cross ratio $t=\operatorname{cr}(0,1, z, \infty)$. Then it is easy to see that the cycle $\alpha \times \beta \times\left(\nu \cap F_{z}\right) \times M_{0}$ is transverse to the image of $\overline{\mathcal{M}}_{4}^{\nu}$. Hence every point counted in $n_{P, \chi}(a, b, v,[M] ; \sigma)$ is also counted in $n_{P}(a, b, v \cap[M],[M] ; \sigma)$. Moreover, because the classes $a, b, v \cap[M],[M]$ are all represented by vertical cycles, the converse also holds.

Part (iv) should be considered as the analog of (iii) for nonvertical classes. $n_{P}(v, a, b ; \sigma)$ counts the intersection of $\overline{\mathcal{M}}^{\nu}=\overline{\mathcal{M}}_{0,3}^{\nu}(P, J, \sigma)$ with a representative of $v \times a \times b$. This time, put the representatives $\alpha, \beta$ for $a, b$ in the same fiber. Then the intersections of $e v\left(\overline{\mathcal{M}}^{\nu}\right)$ with the representing cycle for $v \times a \times b$ all occur in the image of the codimension 2 subcycle $S$ of stable maps with $\geq 2$ components. Moreover, we are interested only in that part $S^{\prime}$ of $S$ on which the last two marked points $z_{2}, z_{3}$ lie on the same branch. Then if $(P \times P)_{\Delta}$ denotes the inverse image of the diagonal $\Delta$ under the map $P \times P \rightarrow S^{2} \times S^{2}$, there is an evaluation map

$$
e v^{\prime}: S^{\prime} \rightarrow P \times(P \times P)_{\Delta},
$$

and it follows from Proposition 1.4, that $n_{P}(v, a, b ; \sigma)$ is precisely the number of intersection points of $e v\left(S^{\prime}\right)$ with $\nu \times \alpha \times \beta$ when this is considered as a cycle in $P \times(P \times P)_{\Delta}$. There are two kinds of curves counted here: one with $z_{1}$ on the stem and the other with $z_{1}$ on the same branch as $z_{2}, z_{3}$. The first kind add up to the first sum:

$$
\sum_{B, i} n_{P}\left(v,[M], e_{i} ; \sigma-B\right) \cdot n_{M}\left(f_{i}, a, b ; B\right)
$$

and the second kind to the second sum:

$$
\sum_{B, i} n_{P}\left([M],[M], e_{i} ; \sigma_{B}\right) \cdot n_{M}\left(f_{i}, v, a, b ; B\right) .
$$

The argument here follows the usual proof of the associativity rule for the quantum product (see [MS] $\S 8.2$, for example.) The point is that one wants to count intersecting pairs of curves in $P$, one in class $\sigma-B$ and the other in class $B$. Thus one is looking at the intersection of the moduli space of all such pairs of curves with the diagonal in $P \times P$. But the diagonal lies in the homology class $\sum_{i} e_{i} \times s\left(f_{i}\right)+s\left(e_{i}\right) \times f_{i}$. One now checks that in both cases the intersection with the classes $s\left(e_{i}\right) \times f_{i}$ is zero. Moreover, the intersection with $e_{i} \times s\left(f_{i}\right)$ is in the first case just

$$
\sum_{B} n_{P}\left(v,[M], e_{i} ; \sigma-B\right) \cdot n_{M}\left(f_{i}, a, b ; B\right) .
$$

The second case is similar, except that here one needs to use a version of Proposition 1.6(ii) that takes account of the fact that we are working in $P \times(P \times P)_{\Delta}$ instead of $P^{3}$. Further details are left to the reader.

\section{Proof of Claim 2.11}

Here is a restatement of this claim in our current language. The statement below makes sense because the map $g r$ extends to a neighborhood of $\overline{\mathcal{M}}_{0,3}\left(M, B, J_{M}\right)$ in the space of stable maps, and hence in particular to $\overline{\mathcal{M}}_{0,3}^{\nu}\left(M, B, J_{M}\right)$. 
Lemma 4.15 Given any regularization

$$
\overline{\mathcal{M}}^{\nu}=\overline{\mathcal{M}}_{0,3}^{\nu}\left(M, B, J_{M}\right)
$$

of $\overline{\mathcal{M}}_{0,3}\left(M, B, J_{M}\right)$, the invariant $n_{P}\left(a, b ; \sigma_{0}+B\right)$ is the intersection number of ev $\operatorname{gr}\left(\overline{\mathcal{M}}^{\nu}\right)$ with a generic cycle in $M \times M$ representing $a \times b$.

Proof: Fix two fibers $M_{0}, M_{1}$ of $P$ and let $\mathcal{W}^{\prime}$ be the space of stable maps $\tau=\left[\Sigma, h, z_{0}, z_{1}\right]$ for which $h\left(z_{i}\right) \in M_{i}$. We claim that the branched pseudomanifold $\operatorname{gr}\left(\overline{\mathcal{M}}^{\nu}\right)$ regularizes the moduli space

$$
\mathcal{M}^{\prime}(P)=\overline{\mathcal{M}}_{0,2}\left(M \times S^{2}, \sigma_{0}+\iota(B) \cap \mathcal{W}^{\prime} . J\right)
$$

To see this, first observe that the expected or formal dimension of $\mathcal{M}^{\prime}(P)$ is

$$
2 n+2+2 c_{1}\left(\sigma_{0}+B\right)-6=2 n+2 c_{1}(B)=\text { f. } \operatorname{dim} . \mathcal{M}_{0,3}(M, B, J),
$$

where $6=\operatorname{dim} \operatorname{PSL}(2, \mathbf{C})$ is subtracted because, if the marked points lie on the stem component and if this is parametrized as a graph, the position of the marked points is uniquely determined. (As a check, note also that if a marked point $z_{i}$ is in a branch component, this component is constrained to lie in the chosen fiber $M_{i}$ and so the two extra dimensions from the possible movement of $z_{i}$ are cancelled out by this codimension 2 constraint on the branch.) Hence $\operatorname{gr}\left(\overline{\mathcal{M}}^{\nu}\right)$ does have the right dimension. Next, observe that the images by $g r$ of the choices (for example of coverings $U_{i}, \widetilde{V}_{I}$ ) made in the construction of $\overline{\mathcal{M}}^{\nu}$ are valid choices for the construction of a regularization of $\mathcal{M}^{\prime}(P)$. This proves the claim.

It remains to check that $n_{P}(\iota(a), \iota(b) ; \sigma)$ can be calculated from this regularization via the evaluation map into $M_{0} \times M_{1}$. But this was proved in Lemma 4.14.

This lemma immediately implies that $n_{P}\left(a, b ; \sigma_{0}+B\right)$ is always 0 . The basic reason is that $e v \circ g r_{*}\left(\overline{\mathcal{M}}^{\nu}\right)$ reduces dimension: $\operatorname{dim}\left(e v \circ g r_{*}\left(\overline{\mathcal{M}}^{\nu}\right)\right)$ is strictly smaller than $\operatorname{dim}\left(\overline{\mathcal{M}}^{\nu}\right)=$ $2 n+2 c_{1}(B)$ since the image of $\tau$ does not change when the marked point $z_{2}$ is moved in its given component. To be more precise, note that $n_{P}\left(a, b ; \sigma_{0}+B\right) \neq 0$ only when

$$
\operatorname{dim}(a)+\operatorname{dim}(b)+2 c_{1}(B)=2 n .
$$

Further the intersection points of $e v \circ g r_{*}\left(\overline{\mathcal{M}}^{\nu}\right)$ with $a \times b$ are precisely those of

$$
e v_{M}\left(\overline{\mathcal{M}}_{0,2}^{\nu}\right) \rightarrow M \times M
$$

with $a \times b \times M$. But the image of $e v_{M}$ has codimension $2 n-2 c_{1}(B)-2$ in $M \times M$ and so one can move $a \times b$ to be disjoint from this image.

We end this subsection by sketching a proof of Lemma 3.24 which says:

Lemma 4.16 Let $P=M \times S^{2}$ and $\sigma_{0}$ be the section class $\left[p t \times S^{2}\right]$. Then, for all $a \in H_{*}(M)$ and $u, v, w \in H_{*}(P)$,

(i) $n_{P}(u, v, w ; D)=0$ unless $D=k \sigma_{0}+B$ for some $B \in H_{2}(M)$ and $k=0,1$.

(ii) $n_{P}\left(s(a), u, v ; \sigma_{0}+B\right)=0$. 
Proof: The proof of (ii) is very similar to that of Lemma 4.15. Let us first consider

$$
n_{P}\left(a, s(b), s(c) ; \sigma_{0}+B\right) \text {. }
$$

This time, because only one of the classes $a, s(b), s(c)$ is in $H_{*}(M)$, we fix one fiber $M_{0}$ and let

$$
\mathcal{W}^{\prime}=\left\{\tau=\left[\Sigma, h, z_{0}, z_{1}, z_{2}\right]: h\left(z_{0}\right) \in M_{0}\right\}
$$

Further define

$$
g r^{\prime}: \overline{\mathcal{M}}_{0,5}\left(M, B, J_{M}\right) \rightarrow \overline{\mathcal{M}}_{0,3}\left(M \times S^{2}, \sigma_{0}+B, J\right) \cap \mathcal{W}^{\prime}
$$

by setting

$$
g r^{\prime}\left(\left[\Sigma, h, z_{0}, \ldots, z_{4}\right]\right)=\left[\Sigma, \widehat{h}, z_{0}, z_{3}, z_{4}\right]
$$

where, as in the definition of $g r$,

$$
\widehat{h}(z)=(h(z), \gamma(z)) \in M \times S^{2}
$$

is defined using the first three marked points $z_{0}, z_{1}, z_{2}$. As before, it is not hard to check that if $\overline{\mathcal{M}}^{\nu}$ regularizes $\overline{\mathcal{M}}_{0,5}\left(M, B, J_{M}\right)$, then $g r^{\prime}\left(\overline{\mathcal{M}}^{\nu}\right)$ regularises $\overline{\mathcal{M}}_{0,3}\left(M \times S^{2}, \sigma_{0}+B, J\right) \cap \mathcal{W}^{\prime}$ in $\mathcal{W}^{\prime}$. Moreover, there is an evaluation map

$$
e v^{\prime}: g r^{\prime}\left(\overline{\mathcal{M}}^{\nu}\right) \rightarrow M \times P \times P \text {. }
$$

Clearly, $e v^{\prime}\left(g r^{\prime}(\tau)\right)$ intersects $a \times s(b) \times s(c)$ exactly when $h\left(z_{0}\right) \in a, h\left(z_{3}\right) \in b, h\left(z_{4}\right) \in c$. But $n_{P}\left(a, s(b), s(c) ; \sigma_{0}+B\right) \neq 0$ only when

$$
2 n+2+4+2 c_{1}(B)+\operatorname{dim}(a)+\operatorname{dim}(b)+\operatorname{dim}(c)+4=6 n+6 .
$$

i.e. if $2 c_{1}(B)+\operatorname{dim}(a)+\operatorname{dim}(b)+\operatorname{dim}(c)=4 n-4$. But in these dimensions there are for generic $J$ no $J$-curves in $M$ through $a, b, c$. More precisely, representatives for $a, b, c$ (still called $a, b, c)$ can be chosen in $M$ so that the image of

$$
e v: \overline{\mathcal{M}}_{0,3}\left(M, B, J_{M}\right) \rightarrow M \times M \times M
$$

does not meet $a \times b \times c$.

Similar arguments show that $n_{P}(s(a), b, c ; \sigma)=n_{P}(s(a), s(b), s(c) ; \sigma)=0$ whenever $\sigma=$ $\sigma_{0}+B$.

We now have to see that $n_{P}\left(u, v, w ; k \sigma_{0}+B\right)=0$ whenever $k \neq 0,1$. Note first that these invariants vanish when $k<0$, since positivity of intersections with the fibers $[M]$ of $P$ implies that the moduli spaces $\overline{\mathcal{M}}_{0,3}\left(P, k \sigma_{0}+B, J_{M} \times j\right)$ are empty when $k<0$. When $k>1$ the idea again is to show that the evaluation map reduces dimension by constructing elements of $\overline{\mathcal{M}}_{0,3}\left(P, k \sigma_{0}+B, J_{M} \times j\right)$ from elements of $\overline{\mathcal{M}}\left(M, B, J_{M}\right)$ and $\overline{\mathcal{M}}\left(S^{2}, k \sigma_{0}, j\right)$. (By slight abuse of notation we have written $\sigma_{0}$ for the fundamental class of $S^{2}$.) The only problem is to find a replacement for the map $g r$.

Consider the map

$$
\pi=\pi_{M} \times \pi_{S}: \overline{\mathcal{M}}_{0,3}\left(M \times S^{2}, k \sigma_{0}+B, J_{M} \times j\right) \rightarrow \overline{\mathcal{M}}_{0,3}\left(M, B, J_{M}\right) \times \overline{\mathcal{M}}_{0,3}\left(S^{2}, k \sigma_{0}, j\right)
$$


obtained by projection followed by contraction of unstable components, and let $\mathcal{N}$ be its image. This map $\pi$ is injective on the top component, but need not be injective in general. T1

It is easy to check that for each stratum $\mathcal{S}$ in $\mathcal{N}$ the restriction

$$
\pi: \pi^{-1}(\mathcal{S}) \rightarrow \mathcal{S}
$$

is a fibration. Note also that $\overline{\mathcal{M}}_{0,3}\left(S^{2}, k \sigma_{0}, j\right)$ is an orbifold of the correct dimension and so is already regular. (All the relevant maps $D \tilde{h}$ are already surjective.) Consider the pullback

$$
\overline{\mathcal{M}}^{\nu}\left(M \times S^{2}\right)=\pi^{-1}\left(\overline{\mathcal{M}}^{\nu}(M) \times \overline{\mathcal{M}}_{0,3}\left(S^{2}, k \sigma_{0}, j\right)\right.
$$

where $\overline{\mathcal{M}}^{\nu}(M)$ is a regularization of $\overline{\mathcal{M}}_{0,3}\left(M, B, J_{M}\right)$. By the above remarks this is a branched pseudomanifold $Y$. One now has to check that $Y$ is homologous to a regularization of $\overline{\mathcal{M}}_{0,3}\left(M \times S^{2}, k \sigma_{0}+B, J_{M}+j\right)$. Note that $Y$ is made in much the same way as is the regularization. However it is made from a cover of the moduli space that is pulled back from the product of a cover of $\overline{\mathcal{M}}_{0,3}\left(S^{2}, k \sigma_{0}, j\right)$ with $\overline{\mathcal{M}}_{0,3}\left(S^{2}, k \sigma_{0}, j\right)$, and these pullback sets are larger than the sets used in the constructions in $\S 4.2$. However, these constructions behave well when the cover is refined, and, using this, the desired result follows fairly easily.

Granted this, we can use this pullback set $\overline{\mathcal{M}}^{\nu}\left(M \times S^{2}\right)$ when evaluating the GromovWitten invariants for $M \times S^{2}$. Now observe that when $k>1$ the image of the evaluation map

$$
\overline{\mathcal{M}}_{0,3}\left(S^{2}, k \sigma_{0}, j\right) \rightarrow\left(S^{2}\right)^{3}
$$

has dimension strictly smaller than

$$
\operatorname{dim}\left(\overline{\mathcal{M}}_{0,3}\left(S^{2}, k \sigma_{0}, j\right)\right)=2+4 k .
$$

Hence, for dimensional reasons, this evaluation map never gives rise to nonzero GromovWitten invariants. This implies that the evaluation map

$$
\overline{\mathcal{M}}^{\nu}(M) \times \overline{\mathcal{M}}_{0,3}\left(S^{2}, k \sigma_{0}, j\right) \stackrel{e v_{M} \times e v_{S}}{\longrightarrow} M^{2} \times\left(S^{2}\right)^{3}
$$

also has too small an image to give rise to nonzero Gromov-Witten invariants. Hence the same is true for

$$
e v_{M \times S}: \overline{\mathcal{M}}_{0,3}^{\nu}\left(M \times S^{2}\right) \rightarrow\left(M \times S^{2}\right)^{3}
$$

since this map factors through $e v_{M} \times e v_{S}$.

\subsubsection{The composition rule}

It remains to prove Lemma 2.12 which is the last step in the proof of Proposition 2.5. We assume that we are in the situation described in $\S 2.3 .2$. The space $\mathcal{X}$ fibers over the disc $\Delta$ with fibers $X_{t}$ that are themselves fibered. Thus the projection $\mathcal{X} \rightarrow \Delta$ factors as

$$
\mathcal{X} \stackrel{q}{\longrightarrow} \mathcal{S} \stackrel{\pi}{\longrightarrow} \Delta
$$

\footnotetext{
${ }^{11}$ To get injectivity, one must also keep track of information about the domain $\Sigma$ of the element in the left hand side and how it relates to the domains of its projections to $M$ and $S^{2}$. For example, suppose that $\Sigma$ has three components $\Sigma_{1}$, that maps into a fiber, and $\Sigma_{i}, i=2,3$ where $\Sigma_{i}$ maps to the flat section $p_{i} \times S^{2}$. Then the image of this element $\pi$ is independent of the choice of $p_{2}, p_{3} \in M$.
} 
and the construction of the perturbation term $\nu$ must now be compatible with both fibrations. Recall that $\mathcal{S}$ is a holomorphic space and that $\pi: \mathcal{S} \rightarrow \Delta$ is holomorphic with a singular fiber at 0 . This singular fiber is the transverse union of two -1 spheres. Hence all these fibers in $\mathcal{S}$ are regular as far as Fredholm theory goes. It follows easily that the proof of Lemma 4.9 can be adapted to show:

Lemma 4.17 In this situation, we can take the bundle $V$ in Proposition 4.8 to be the vertical tangent bundle $T_{\text {vert }} \mathcal{X}$ of the fibration $\mathcal{X} \rightarrow \mathcal{S}$.

Note that the fiber of $T_{v e r t} \mathcal{X}$ at the point $x \in X_{t}$ can be identified with the fiber of $T_{\text {vert }}\left(X_{t}\right)$ at $x$, in other words it consists of vectors tangent to $M$.

We now construct local uniformizers $\left(\widetilde{U}_{\tau}, \Gamma_{\tau}\right)$ and families of embeddings $e_{\tilde{\tau}^{\prime}}: R_{\tau} \rightarrow \widetilde{L}_{\widetilde{h}^{\prime}}^{V}$ for $\tilde{\tau}^{\prime} \in \widetilde{U}_{\tau}$ as usual, starting first with the points $\tau \in \mathcal{W}\left(X_{0}\right) \subset \mathcal{W}(\mathcal{X})$. The aim is to do this compatibly with the projection $\mathcal{X} \rightarrow \Delta$, i.e. so that the regularization $Y(\mathcal{X})$ can be thought of as a family of regularizations $Y\left(X_{t}\right)$, for $t$ near 0 . To do this one just follows the construction given in Proposition 4.6 and Lemma 4.5 for a codimension 2 subcycle. In other words, make a regularization $Y\left(X_{0}\right)$ and then extend this over a neighborhood in $\mathcal{W}(\mathcal{X})$ by gluing. The family of curves $\Sigma_{t}$ is precisely what one obtains by gluing the singular $\Sigma_{0}$ with parameter $t$. Hence, because the perturbation term $\nu$ projects to 0 in $\mathcal{S}$, when one glues a lift $C_{0} \subset X_{0}$ of $\Sigma_{0}$ with parameter $t$ one gets a curve $C_{t}$ in $X_{t}$.

Therefore, one can construct $Y(\mathcal{X})$ so that, for all sufficiently small $t$, the inverse image $Y\left(X_{t}\right)$ of $\mathcal{W}\left(X_{t}\right)$ in $Y(\mathcal{X})$ is a regularization for $\overline{\mathcal{M}}\left(X_{t}\right)$. Of course $Y(\mathcal{X})$ is not compact, but the restrictions $Y\left(X_{t}\right)$ are all compact.

\section{Proof of Lemma 2.12}

We have now shown how to construct a regularization $Y(\mathcal{X})$ of $\overline{\mathcal{M}}_{0,4}(\mathcal{X}, \widetilde{J}, \widetilde{\sigma})$ that is compatible with the fibration $\mathcal{X} \rightarrow \Delta$. Let $Y_{\chi}(\mathcal{X})$ be the subset on which the cross ratio is fixed. As before $Y_{\chi}(\mathcal{X})$ is a union of virtual moduli cycles $Y_{\chi}\left(X_{t}\right)$.

The first step is to prove that one can achieve transverse intersections of $e v\left(Y_{\chi}(\mathcal{X})\right)$ with the class $\prod\left(\left[\widetilde{\alpha}_{i}\right]\right)$ by choosing generic cycles $\widetilde{\alpha}_{i}$ that are compatible with the fibrations on $\mathcal{X}$ as described in $\S 2.3 .2$. The needed arguments are very similar to those in Lemma 4.14 and will be left to the reader.

Next observe that because of the special form of the cycles $\widetilde{\alpha}_{i}$ the only elements in $Y_{\chi}(\mathcal{X})$ that meet the cycles $\widetilde{\alpha}_{i}$ lie in a single set $Y_{\chi}\left(X_{t}\right)$. Hence one gets an intersection of ev $\left(Y_{\chi}\left(X_{t}\right)\right)$ with the cycle $\prod\left(\widetilde{\alpha}_{i}\right)_{t} \in\left(X_{t}\right)^{4}$, where $(\widetilde{\alpha})_{t}=\widetilde{\alpha} \cap X_{t}$. However this intersection is not transverse, since it can be destroyed by moving the fiber $\left(\widetilde{\alpha}_{2}\right)_{t}=M$ to another fiber in $X_{t}$. If one did this in $\mathcal{X}$ the effect would be to change the relevant value of $t$. It is now not hard to see that one can get a transverse intersection in $X_{t}$ by considering the intersection of $\prod\left(\widetilde{\alpha}_{i}\right)_{t}$ with the image of the whole of $Y\left(X_{t}\right)$ rather than just $Y_{\chi}\left(X_{t}\right)$. This proves (i).

Now consider (ii). Note that the cross-ratio of the 4 marked points is fixed on the component of $Y\left(X_{0}\right)$ on which there are two marked points mapping to each component of $\Sigma_{0}$. Since this is the only component that intersects the cycle $\prod\left(\widetilde{\alpha}_{i}\right)_{0}$, we can think that $Y_{\chi}\left(X_{0}\right)=Y\left(X_{0}\right)$. Moreover, again we can achieve transversality of intersection in $X_{t}$ for all $t$ near 0 by taking generic cycles $\widetilde{\alpha}_{i}$ of the given form.

Because $Y\left(X_{0}\right)$ is a codimension 2 subcycle in $Y(\mathcal{X})$, it follows from Proposition 4.4 that the intersection number $n_{X_{0}}(a,[M],[M], b)$ of $Y\left(X_{0}\right)$ with $\prod\left(\widetilde{\alpha}_{i}\right)_{0}$ is the same as the 
intersection number $n_{X_{t}}(a,[M],[M], b)$ of $Y\left(X_{t}\right)$ with $\prod\left(\widetilde{\alpha}_{i}\right)_{t}$ for each fixed $t$ near 0 . It remains to check that $n_{X_{0}}(a,[M],[M], b)$ is precisely

$$
\sum_{\sigma=\sigma_{1} \# \sigma_{2}} n_{P_{\phi}}\left(a,[M], e_{i} ; \sigma_{1}\right) n_{P_{\psi}}\left(f_{i},[M], b ; \sigma_{2}\right) .
$$

This completes the proof of Lemma 2.12. Note that a very similar argument would prove the composition rule (6) for general Gromov-Witten invariants.

\section{References}

[An] S. Anjos, in preparation.

[B] A. Blanchard, Sur les variétés analytiques complexes, Ann. Sci. Ec. Norm. Sup. , (3) 73, (1956).

[FO] K. Fukaya and K. Ono, Arnold conjecture and Gromov-Witten invariants, preprint (1996)

[Gi] A. Givental, Equivariant Gromov-Witten invariants, Internat. Math. Research Notes, (1996), 613-663.

[G] M. Gromov, Pseudo holomorphic curves in symplectic manifolds, Inventiones Mathematicae, 82 (1985), 307-47.

[HLS] H. Hofer, V. Lizan and J.-C. Sikorav, On genericity for complex curves in 4-dimensional almost complex manifolds, Duke Math Journal,

[HS] H. Hofer and D.A. Salamon, Floer homology and Novikov rings, Floer Memorial volume eds. Hofer, Taubes, Weinstein, Zehnder, Birkhäuser, Basel, (1996).

[HS] H. Hofer and D.A. Salamon, Marked Riemann surfaces of genus 0, preprint (1997).

[IP] E. Ionel and T. Parker, Gromov-Witten invariants of symplectic sums, (in preparation)

M. Kontsevich and Yu. Manin, Quantum Cohomology of a Product.

[LMP1] F. Lalonde, D. McDuff and L. Polterovich, On the Flux conjectures, CRM Proceedings and Lecture Notes vol 15, (1998), 69-85

[LMP2] F. Lalonde, D. McDuff and L. Polterovich, Topological rigidity of Hamiltonian loops and quantum homology, to appear in Invent. Math.

[LMP3] F. Lalonde, D. McDuff and L. Polterovich, On Hamiltonian fibrations, in preparation.

[LO] Hong-Van Le and Kaoru Ono, Topology of symplectomorphism groups and Pseudoholomorphic curves, preprint (1997)

[LiT] Jun Li and Gang Tian, Virtual moduli cycles and Gromov-Witten invariants for general symplectic manifolds, preprint alg-geom 9608032.

[LiuT1] Gang Liu and Gang Tian, Floer homology and Arnold conjecture, Journ. Diff. Geom (1998). 
[LiuT2] Gang Liu and Gang Tian, On the equivalence of multiplicative structures in Floer Homology and Quantum Homology, preprint Oct.1998.

[Mc1] D. McDuff, From deformation to isotopy, to appear in Proceedings of the 1996 Conference at Irvine, ed. Stern, International Press.

[Mc2] D. McDuff, Almost complex structures on $S^{2} \times S^{2}$, SG/9808008, to appear in Duke Math. Journal.

[Mc3] D. McDuff, The virtual moduli cycle, preprint (1998).

[Mc4] D. McDuff, Fibrations in Symplectic Topology, Documenta Mathematica, Extra Volume ICM 1998, Vol I, 339-357.

[MS1] D. McDuff and D.A. Salamon, J-holomorphic curves and quantum cohomology, Amer Math Soc Lecture Notes \#6, Amer. Math. Soc. Providence (1994).

[MS2] D. McDuff and D.A. Salamon, Introduction to Symplectic Topology, 2nd edition, OUP, (1998).

[PSS] S. Piunikhin, D. Salamon and M. Schwarz, Symplectic Floer-Donaldson theory and Quantum Cohomology, Contact and Symplectic Geometry ed C. Thomas, Proceedings of the 1994 Newton Institute Conference, CUP, Cambridge (1996)

[P] L. Polterovich: Symplectic aspects of the first eigenvalue, preprint (1997)

[R] Y. Ruan, Virtual neighborhoods and pseudoholomorphic curves, preprint 1996

[RT] Y. Ruan and G. Tian, A mathematical theory of quantum cohomology. Journ Diff Geo 42 (1995), 259-367.

[Sd1] P. Seidel, $\pi_{1}$ of symplectic automorphism groups and invertibles in quantum cohomology rings, Geometric and Functional Analysis, 7 (1997), 1046-95.

[Sd2] P. Seidel, On the group of symplectic automorphisms of $\mathbf{C} \mathbf{P}^{m} \times \mathbf{C P}^{n}$, preprint (1998)

[Sb] B. Siebert, Gromov-Witten invariants for general symplectic manifolds, preprint (1997) 